1993

\title{
Structural Incongruity and Humor Appreciation
}

Rosemary Evans Staley

College of William \& Mary - Arts \& Sciences

Follow this and additional works at: https://scholarworks.wm.edu/etd

Part of the Cognitive Psychology Commons

\section{Recommended Citation}

Staley, Rosemary Evans, "Structural Incongruity and Humor Appreciation" (1993). Dissertations, Theses, and Masters Projects. Paper 1539625847.

https://dx.doi.org/doi:10.21220/s2-esfb-v242

This Thesis is brought to you for free and open access by the Theses, Dissertations, \& Master Projects at W\&M ScholarWorks. It has been accepted for inclusion in Dissertations, Theses, and Masters Projects by an authorized administrator of W\&M ScholarWorks. For more information, please contact scholarworks@wm.edu. 
STRUCTURAL INCONGRUITY AND HUMOR APPRECIATION

\begin{abstract}
A Thesis
Presented to

The Faculty of the Department of Psychology

The College of William and Mary, in Virginia
\end{abstract}

In Partial Fulfillment

of the Requirements for the Degree of

Master of Arts

by

Rosemary Evans staley

1993 
APPROVAL SHEET

This thesis is submitted in partial fulfillment of the requirements for the degree of

Master of Arts
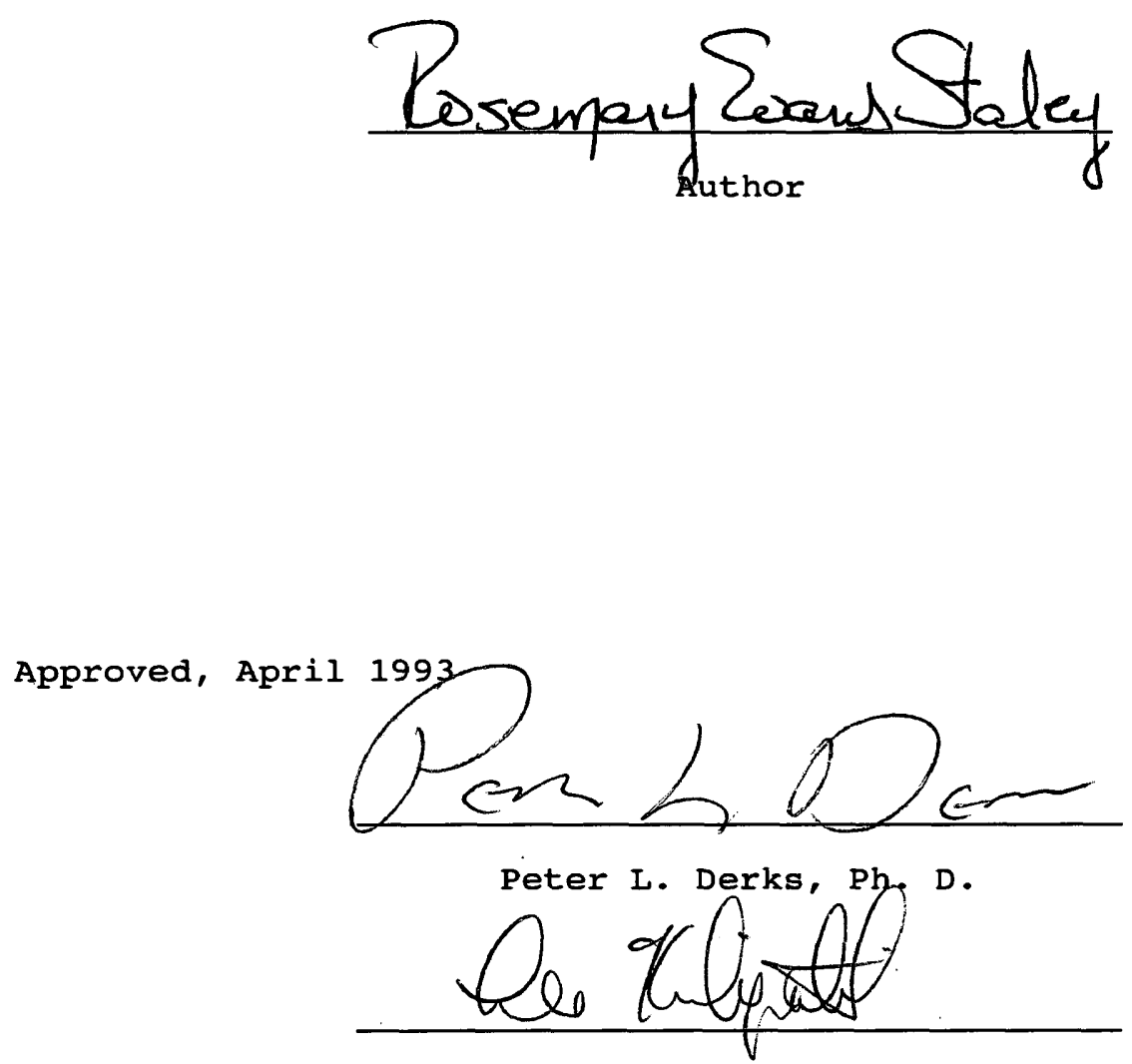

Lee A. Kirkpatrick, Ph. D.

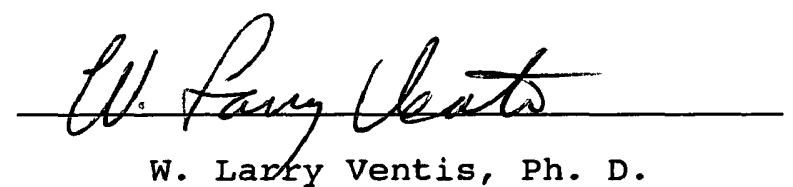

W. Lanky Vents, Ph. D.

ii 
This thesis is dedicated to Allan staley whose love, understanding, and good humor throughout the term of this project made its completion possible. 
INTRODUCTION. ...............................

COGNITIVE STAGE MODELS OF HUMOROUS STRUCTURAL

INCONGRUITY .....................................

SIMULTANEITY AND COMPREHENSION.......................

EMOTIONAL TOLERANCE.............................

INDIVIDUAL DIFFERENCES IN EMOTIONAL TOLERANCE.............. 9

COGNITIVE TOLERANCE................................ 11

CONTEXT AS A SOCIAL VARIABLE......................... 12

CONTEXT AS A COGNITIVE VARIABLE....................... 13

CORRELATIONAL STUDY.............................. 16

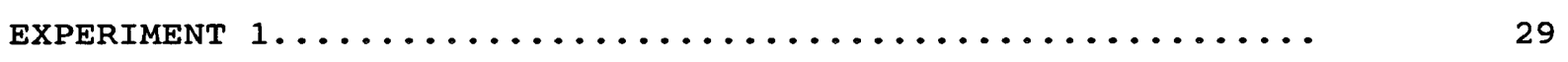

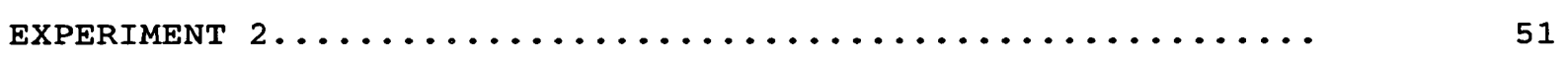

GENERAL DISCUSSION. ............................. 80

REFERENCES ......................................

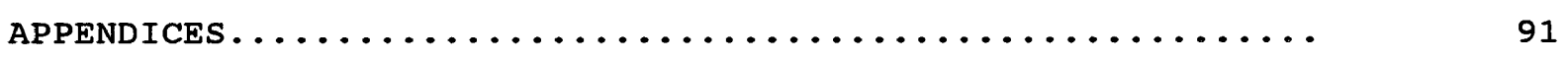

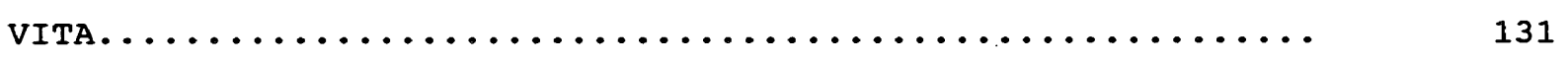


The author wishes to express her gratitude to Professor Peter Derks for both his introducing her to the psychological study of humor and his infinitely patient guidance and sage criticism throughout each stage of this project. She would also like to thank Professor Lee Kirkpatrick for his encouraging suggestions, helpful criticisms and statistical advice. The author is also thankful to Professor Larry Ventis for his conducive recommendations and criticisms. The author would also like to acknowledge her parents, Christine and David Evans, for first instilling and then nurturing in her a love for knowledge that led her to be curious about the nature of things. 
Table

1. Correlation Matrix of Nonsense and Incongruity Resolution for Funniness and Aversiveness and Personality Variables.......................

2. Differences in Funniness and Aversiveness scores of the Humor Categories......................

3. Correlation Matrix of Difference scores for Funniness and Aversiveness and Personality

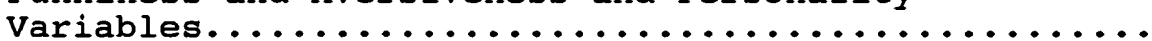

4. Correlation Matrix of Funniness and Aversiveness Sumscores for Nonsense and Personality

Variables...............................

5. Correlation Matrix of Funniness and Aversiveness Sumscores for Incongruity Resolution and Personality Variables................................

6. Experiment 2: Experimental Design..............

7. Mean Funniness Ratings of the Seven Cartoon Trials for the Eight Prime and Switch Manipulation Groups...............................

8. Mean Aversiveness Ratings of the Seven Cartoon Trials for the Eight Prime and Switch Manipulation

Groups............................... 


\section{LIST OF FIGURES}

Figure

Page

1. Mean Funniness Ratings, 36 Trials of Incongruity

Resolution and Nonsense Cartoons................

2. Mean Aversiveness Ratings, 36 Trials of Incongruity

Resolution and Nonsense Cartoons...............

3. Mean Funniness Ratings, Four Trials of Incongruity Resolution and Nonsense Cartoons.................

4. Mean Aversiveness Ratings, Four Trials of Incongruity Resolution and Nonsense Cartoons...............

5. Mean Funniness Ratings, First Four Trials, of Incongruity Resolution and Nonsense Cartoons................

6. Mean Aversiveness Ratings, First Four Trials, of Incongruity Resolution and Nonsense Cartoons........

7. Mean Funniness Ratings, Seven Trials of Incongruity Resolution and Nonsense Cartoons...............

8. Mean Aversiveness Ratings, Seven Trials of Incongruity Resolution and Nonsense Cartoons................ 
Abstract

An overview of research on the cognitive basis of humor is considered including the debate between shultz (1972), Suls (1972), Rothbart and Pien (1977) and Ruch (1992) concerning incongruity as a sufficient (i.e., nonsense) or merely a necessary (i.e., incongruity resolution) stimulus structure for humor. Comprehension and the process variables, simultaneity, tolerance and context are discussed as necessary features of humor appreciation (Bariaud, 1989; Deckers et al., 1989; Derks \& Arora, 1990; Forabosco, 1991; Goldstein et al., 1973; Leventhal \& Safer, 1977; and Ruch, 1992). Study 1 evaluated the role of personality dependent tolerance for ambiguity as a predictor of appreciation for humor structure. In study 1, mixed results show: incongruity resolution was rated as significantly funnier than nonsense; nonsignificant personality predictions for differential appreciation of humor structure; and significant relationships contradicting Ruch's (1992) theoretical relationship between nonsense and incongruityresolution. These findings suggested that an experimental analysis of the effects of structural incongruity on humor appreciation was necessary. Experiments 1 and 2 evaluated the roles of emotional tolerance, structural salience and context effects on humor appreciation. Both experiments indicate that nonsense and incongruity resolution are not only perceived as distinct humor structures, but this structural distinction leads to higher funniness and higher aversiveness ratings for incongruity resolution than for nonsense. Results of experiment 1 indicate that personality may be a moderating variable for structural appreciation when cartoons are presented in a series. Results of experiment 2 demonstrate that the presentation of incongruity resolution in the context of nonsense can significantly decrease appreciation for nonsense. These results are discussed and suggestions are made for further evaluation of the effects of both personality and the context created by structural incongruity on appreciation for humor. viii 
STRUCTURAL INCONGRUITY AND HUMOR APPRECIATION 
The human capacity to appreciate certain events as humorous has evolved from a topic of philosophical fascination to an area of diverse empirical inquiry. The philosophical concept of incongruity, the simultaneous occurrence of normally incompatible elements, is retained as a central feature of psychological humor theory. Some humor researchers focus on demonstrating that pure incongruity is a sufficient element for the construction of potentially humorous stimuli (Rothbart \& Pien, 1977, and Ruch, 1992); others maintain that incongruity is a necessary but not a sufficient feature of humorous stimuli because resolution is also required in order to complete conceivably humorous stimuli (Shultz, 1972; and Suls, 1972). A majority of researchers concentrate on delineating and discovering the functions of the particularities of the presentation of humor content and structure (Deckers, Buttram \& Winsted, 1989; Derks \& Arora, 1993; Forabosco, 1991; and Goldstein, suls \& Anthony, 1972), the various roles of comprehension and the processing factors, simultaneity, tolerance and context (Bariaud, 1989; Leventhal \& Safer, 1977; McGhee, 1979; Morreall, 1989; Nerhardt, 1977; and Wyer \& Collins, 1992), and the influence of individual differences in the elicitation of the humorous response (Ruch, 1986a, 1986b, 1988, 1992; and Ruch, Ott \& Bariaud, 1991). The assortment of variables hypothesized as responsible for the appreciation of humor indicates that a complexity of potential interactions between cognitive processes, emotional reactions, personality characteristics, and situational factors must finally be explained in a comprehensive theory of humor appreciation.

Incongruity is emphasized as the primary concept in cognitive views of humor because the particular presentation of incongruity constitutes the structure of the humorous stimulus. A Gestalt description defines how incongruity operates as humor (McGhee, 1979 ). "In a Gestalt view, the meaning of an element or group of elements depends upon the whole configuration of which it is a part. Changing 
only a single part of the whole may change the meaning not only of the part, but of the whole as well" (McGhee, 1979, p. 11). For example, in the joke "how many academics does it take to change a lightbulb ?" the humor recipient is presented with the incongruous relationship between an academic and changing a lightbulb. The structure of the joke primes the recipient to believe that there is a funny but meaningful answer to the question which both involves a certain number of academics and clarifies the relationship between the academic and the changing of a lightbulb. The punchline, "none, that is why they have graduate students" makes the meaning of the incongruous relationship between an academic and changing a lightbulb surprisingly explicit by switching the focus of the joke to center instead on the relationship between academics and their graduate students.

In humor appreciation, the unexpected meaning of one element changes the meaning of the whole, and the new meaning of the focal element is accomplished. through the structural reversal of figure and ground (McGhee, 1979). "The structure of the joke draws attention to certain elements while deemphasizing others, forming a background or setting for the apparent focal points of the joke" (McGhee, 1979, p. 12). In the previous example, the structure of the joke highlights the incongruous relationship between an academic and changing a lightbulb. When figure and ground are reversed, the point of the joke unfolds. Bringing in a new concept of the graduate student changes the focus of the relationship between an academic and changing a lightbulb. The punchline unexpectedly brings the background material to the center of attention (McGhee, 1979). Introducing the idea of the graduate student alters the importance of the lightbulb from a central idea to an accessory concept. Changing the relevance of the lightbulb serves as a vehicle to redirect the focus of the joke to highlight the humorously constructed relationship between the academic and the graduate student. The example joke is defined structurally as incongruity resolution. 
Both shultz (1972) and Suls (1972) maintain that both an incongruity and a humorously understandable resolution of the incongruity are the necessary features of the potentially humorous stimulus.

Cognitive Stage Models of Humorous Structural Incongruity

Shultz (1972) defines incongruity and resolution as the two necessary stages of structural incongruity required for a potentially humorous response. In this model, an incongruity is defined as "the simultaneous presence of two or more habitually incompatible elements, where an element can be either an object or an event" (Shultz, 1972, p. 457). Based on an individual's knowledge and experience, the juxtaposition of incongruous elements will more or less violate the individual's expectation of congruity. The surprise or cognitive arousal that results from perceiving the incongruity is assumed to be perplexing but, not by itself, humorous (Berlyne, 1972). The humor recipient attempts to justify how the seemingly incongruous elements can in fact meaningfully co-exist. A resolution renders incongruity coherent without making it serious (Bariaud, 1989). According to shultz (1972), a humorous response will follow only if the incongruity can be resolved meaningfully.

Suls (1972) further specifies this two-stage process of initial incongruity followed by a resolution in an information-processing model. In the first stage, an incongruity, a violation of expectation, is detected, and then in the second stage a problem solving search is undertaken to reconcile the punchline with the initial incongruity. "Resolution is the complement of identification of incongruity, and together they make up comprehension, the intellectual part of the humor reaction which is an indispensable basis for amusement" (Bariaud, 1989, p. 20). Such resolution is achieved when a "cognitive rule", a logical proposition, a definition, or a fact of experience, reunites the punchline with the body of the joke. "The retrieval of such information makes it possible to reconcile the incongruous parts of the joke" (Suls, 
1972, p. 82). In the two-stage models (Schultz, 1972; Suls, 1972), incongruity is a necessary but not a sufficient condition for humor. Resolution is the critical complement to incongruity required for a humorous response.

Rothbart and Pien (1977) suggest that incongruity-resolution models do not exhaust the possibilities of humor structures. In some humor stimuli, resolution may be absent, partial or bizarre. The notion of incomplete resolution as potentially humorous adds the concept of nonsense humor to the possible humor structures. Rothbart and Pien (1977) suggest that several categories of incongruity and resolution must be delineated in order to explain the range of structural mechanisms of humor appreciation.

Incongruity entails elements that are unexpected and also impossible given one's knowledge of the world; possible incongruity involves elements that are unexpected or improbable, but possible; complete resolution occurs when the initial incongruity follows completely from resolution information; and, incomplete resolution happens when the initial incongruity follows in some way, but is not completely resolved because the situation remains impossible (Rothbart \& Pien, 1977, p. 37) .

These distinctions indicate that the degree of resolution offered by an incongruous stimulus may range from complete and meaningful to absent or bizarre. Humor appreciation may result from either the meaningful but humorous incongruity-resolution structure or the nonsensical but amusing pure incongruity structure.

Cognitive processing models of humor appreciation thus explain how to present incongruity in a potentially humorous form. These structures therefore constitute the model of the likely humorous stimulus, but they only potentiate a humorous response. Appreciation of the incongruous structure as funny requires a particular perception of the stimulus. A first condition of incongruity models of humor appreciation is that 
comprehension of the conflicting ideas evident in an incongruous stimulus must precede appreciation (Bariaud, 1989). In the "academic changing a lightbulb" joke, knowledge of the relationship between academics and graduate students is required in order to appreciated what makes the joke funny. However, comprehension is not sufficient for the appreciation of incongruity as humorous.

The incongruity between the ideas in a joke will be found amusing only if several necessary conditions beyond comprehension are met. Cognitive, emotional and social factors determine whether an incongruous stimulus will elicit a humorous response. In the "academic changing the lightbulb" joke, the difference between comprehending the joke, understanding why the joke should be amusing and actually perceiving it as funny are dependent upon a particular relationship between the processing factors: "comprehension" (Bariaud, 1989), "simultaneity", "tolerance" and "context" (Leventhal \& Safer, 1977). The apparently incompatible concepts must be accepted as being somehow related, and thus be perceived as occurring "simultaneously" (i.e., involving perhaps unrealistic but nonetheless coherent relationships). "Emotional tolerance" is partly a recognition of the intent of humor or a perception that the joke is not maliciously directed towards academics. This processing factor is critical because incongruity can also lead to responses of negative emotion and puzzlement (Morreall, 1989). For example, mystery stories are rife with incongruities that lead to puzzlement instead of amusement, likewise, not understanding how to construct a bicycle from a selection of parts can lead to frustration instead of laughter. However, resolution of either a mystery or successful completion of a bicycle may each lead to a positive emotion akin to amusement. Future research could assess the relationship between the humorous response and the positive emotions associated with the resolution of incongruity that is not intended to be humorous. An "emotional tolerance" is not necessarily a pre-requisite for 
understanding the joke, but it must be met if the stimulus is to be processed as funny. "Emotional tolerance" also involves the requirement that the contradictions or ambiguity between the incongruous elements involve concepts and relationships between concepts that are affectively admissible. "Emotional tolerance for ambiguity" determines the relative preference for humor structure (Ruch, 1992). The "social context" in which the joke is presented must also be both appropriate and conducive to the appreciation of humor. Finally, a "cognitive context" is created by multiple presentations of jokes, and not only where the "academic changing a lightbulb" joke is located in that series, but also the nature of surrounding jokes can influence the relative funniness of the particular joke (Derks \& Arora, 1993). A comprehensive theory of humor appreciation will include these processing variables; explain how the two types of structural incongruity are perceived and processed as humorous; predict how humor content affects humor appreciation; and define what conditions will render the various combinations of content and structure relatively both more and less amusing.

\section{Simultaneity and Comprehension}

Simultaneity specifies that "in a joke or cartoon, the multiple and incongruous aspects of the joke or cartoon must form a structured whole, with the contrasting meanings bound together and viewed simultaneously" (Leventhal \& Safer, 1977, p. 340). For Suls (1972), Shultz (1977), and McGhee (1979) simultaneity is also a critical factor in humor appreciation representing a playful acceptance of impossible or improbable incongruous elements as a related unit. Morreall (1989) suggests that thinking in non-practical ways allows for potential humor appreciation because the unified elements of humorous incongruity transcend the possibilities of rational thought. Presupposing that an incongruous stimulus must be accepted as a coherent unit implies that it must be understood in a particular fashion. Nerhardt (1977) defines humor as "a consequence of the discrepancy between two mental 
representations, one of which is an expectation and the other is some other idea or percept" (p. 47). For Nerhardt (1977), humor results from the degree of divergence of an event from an expectation, and as the divergence increases so does the funniness. Comprehension of the intended bizarre and simultaneously cohesive relationship between the ideas in an incongruous stimulus is required for a potentially humorous response to the incongruity. Comprehension and simultaneity are cognitive pre-requisites for humor appreciation. However, they are necessary but not sufficient conditions for humor appreciation because they neither guarantee an affective acceptance of incongruity as funny, nor determine the relative funniness of incongruity that is the function of either the social or cognitive context of humor presentation. Emotional Tolerance

Humor appreciation is conceptualized as a phenomenon with both cognitive and emotional pre-requisites (Bariaud, 1989). Incongruity is a potentially funny contradiction or a discrepancy between an event and an expectation (Nerhardt, 1977). However, incongruity does not necessarily elicit a humorous response. Instead, there are three general emotional reactions to incongruity: negative emotion; puzzlement; and, amusement (Morreall, 1989). In negative emotion and puzzlement tension and discomfort with the incongruity serve as motivating factors to change the current conditions in order to remove the anxiety created by the incongruity. By contrast, in amusement the perception of contradiction is not only an acceptable but also an enjoyable state of affairs. Any motivation in amusement is either to sustain or to share the experience.

The process variable, emotional tolerance, complements the cognitive processes of simultaneity and comprehension (Leventhal \& Safer, 1977). Bariaud (1989) defines the affective component of humor appreciation as "emotional complicity", or the process of adopting and sharing the intent of the joke or cartoon. McGhee (1979) refers to 
tolerance as an ability to engage in a "playful frame of mind". An affective distance from the norms of reality or a leap of faith into fantasy is required before one will process incongruity as humor. A tolerance for incongruity transcends cognitive understanding and acceptance of coherence by involving an emotional ability to view incongruity as humorous rather than frightening or serious.

\section{Individual Differences in Emotional Tolerance}

Defining emotional tolerance as a necessary pre-requisite for humor appreciation highlights the role of individual differences in the appreciation of humor. Although several people may appreciate the same joke or cartoon, each individual has a unique sense of humor depending upon variations in experience and knowledge. These individual differences, evident in personality based tolerance for ambiguity, influence what type of structural incongruity the individual will find amusing (Ruch, 1992). Individual differences in tolerance for ambiguity or the degree of divergence between event and expectation (Nerhardt, 1977) may determine when incongruity is perceived as funny, when it is found confusing, and when it is understood as serious.

Ruch and Hehl (1986a, 1986b) and Ruch (1988, 1991, 1992) operationalize a hypothesized personality based emotional tolerance for ambiguity by combining Conservatism (Wilson, 1973) and sensation Seeking (Zuckerman, 1979) as predictors for a relative appreciation for the onestage process, nonsense (NON), or the two-stage process, incongruityresolution (INC-RES). The personality variables are predictors for relative preference because Ruch (1992) reports that INC-RES is the overall preferred humor structure. However, relative differences in appreciation for INC-RES and NON are predictable by variation in emotional tolerance for ambiguity (Ruch, 1992). The Conservative (Wilson, 1973) only enjoys incongruity when it is resolved, and the Sensation seeker (Zuckerman, 1979) also likes incongruity when it is left nonsensical (Ruch, 1992). The relationship between these 
personality variables and a relative structural preference offers a possible explanation of the role of the affective component of tolerance of ambiguity in cognitive appreciation of humor. Unlike Shultz (1972) and Suls (1972), these researchers agree with Rothbart and Pien (1977) and conclude that incongruity may be either a necessary or a sufficient condition for humor depending upon the individual sense of humor. Conservatism is defined as an intolerance of ambiguity, a preference for stability, and a fondness of simplicity, in short, a generalized fear of uncertainty (Wilson, 1973). Sensation seeking, on the other hand, is described as a tolerance of ambiguity, a desire for novelty and change, and an affinity for complexity (Zuckerman, 1979). In Ruch's (1992) individual differences model, independent of joke or cartoon content, Conservatism (Wilson, 1973) and Sensation seeking (Zuckerman, 1979) predict the relative preference for humor structure. Ruch (1992) concludes from a series of factor analytic studies (Ruch \& Hehl, 1986a, 1986b; Ruch, 1988), that it is the level of certainty offered by each structure that appeals to an individual's basic need for either predictability or novelty, and thus determines the relative structural appreciation.

Both building on Rothbart's and Pien's (1977) distinction that the degree of resolution offered in jokes and cartoons is important, and incorporating Nerhardt's (1977) notion that humor arises from an increasing divergence between event and expectation, Ruch (1988) suggests that individual differences in appreciation for structure are dependent on each structure's predictability and degree of solution. Ruch (1992) proposes that because NON embodies uncertainty, it is both more arousing than INC-RES, and requires a higher tolerance for ambiguity in order to be found amusing. INC-RES has an intended resolution, but NON may. either remain incongruous, offer partial resolution, or even create new and bizarre incongruities (Rothbart \& Pien, 1977). 


\section{Cognitive Tolerance}

The Conservative (Wilson, 1973) and the Sensation seeker.

(Zuckerman, 1979) appear to differ in an emotional tolerance for

ambiguity. Perhaps they also vary in a cognitive tolerance for

ambiguity. Comprehension of the unusual relationship between an event and an expectation (Nerhardt, 1977) is assumed to precede appreciation (Bariaud, 1989). Understanding incongruity requires determining the nature of the strange connections of simultaneity. "Freud argued [over] seventy-five years ago that a part of the pleasure derived from humor merely results from exercising the intellect in trying to understand a joke (McGhee, 1979, p. 10)." If humor appreciation is understood as a unique problem solving process (Suls, 1972; Wyer \& Collins, 1992) then, the amount of cognitive effort a person is willing to exert in order to understand the incongruous relationships in a joke or cartoon may also influence individual appreciation for humor structure.

A distinction between the Conservative (Wilson, 1973) and the Sensation seeker (1979) is a relative rigidity or flexibility in attitudes. The conservative desires to maintain his or her existing expectations or attitudes (Wilson, 1973), and the sensation seeker aims to surprise or challenge his or her expectations or attitudes (Zuckerman, 1979). Distinguishing between the personality categories in terms of cognitive effort may be predicted by "The Need for Cognition" scale (Cacioppo \& Petty, 1982) which evaluates the individual dispositional tendency to enjoy and engage in effortful thought.

"People high in need for cognition make more discriminating judgements" (Petty \& Cacioppo, 1986, p. 150) because they enjoy relatively effortful cognitive tasks, even without feedback (Petty \& Cacioppo, 1986). Individuals with a high need for cognition pay more attention to both the structure of concepts and the relationships between ideas (Petty \& Cacioppo, 1986). The amount of thought involved in attitude formation may be a general indicator of how much attention 
an individual is willing to pay to contradictory or at least discrepant ideas. Funniness increases as the discrepancy between the expectation and the event increases (Nerhardt, 1977), and the enjoyment derived from the effort involved in this experience may predict how much discrepancy is funny for different individuals. Individual differences in openness to ideas may be conceptualized as a cognitive tolerance for ambiguity. If an enjoyment in thinking is a variable underlying humor appreciation, then cognitive tolerance for ambiguity may establish a means of assessing Nerhardt's (1977) assertion that increase in discrepancy between an event and an expectation in terms of what is pleasurable for the Conservative (Wilson, 1973) and the Sensation Seeker (Zuckerman, 1979) as humorous information processors.

Above some minimal level of affective tolerance, some jokes and cartoons offer the potential for elaboration without resolution. Nonsense humor offers such stimulation. In the 3WD humor scale (Ruch, 1983), all eighteen of the NON items, but only seven of the INC-RES items, are in cartoon form. It is possible that in general, visual stimuli are more thought provoking than verbal stimuli because they offer less concrete information, and this alone may make NoN more intellectually arousing. The potentially infinite number of ways that visual and nonsensical incongruities may be resolvable could offer intellectual stimulation to the person with a high need for cognition. The purpose of the current correlational study was both an attempted replication of Ruch's (1992) predictions concerning personality based tolerance of ambiguity and a relative preference for humor structure, and an assessment of the Need for Cognition (Cacioppo \& Petty, 1982) as a predictor of structural humor appreciation.

\section{Context as a Social Variable}

Humor appreciation not only depends upon simultaneity, comprehension and a tolerance for incongruity but also hinges upon context. This processing variable is conceptualized as both a social 
variable related to both simultaneity and tolerance (Leventhal \& Safer, 1977), and a cognitive variable dependent upon the particular configuration of a series of jokes and cartoons (Forabosco, 1991). As a social variable, humor may be experienced when incongruity is processed in a safe context where cues indicate that the incongruity is supposed to be funny (Bariaud, 1989). Social context cues such as the wry smile or the glint in a joke teller's eye prime the humor recipient to the fact that humor is both intended and suggests that a laugh or a smile is the appropriate response (McGhee, 1979). This social context is termed "fantasy assimilation" or the recognition of the intent of humor (McGhee, 1979). Social context cues indicate that affective distance or suspended belief is not only appropriate but expected. Incongruity is thus perceived as humorous in a particular social context. However, the social conceptualization of context does not exhaust the possibilities of context cues that can prime the humor recipient to the intent of humor. The cognitive context of humor presentation also influences the appreciation of humor.

\section{Context as a Cognitive Variable}

Context is cognitively conceptualized as a characteristic of the configuration or the serial position of the humor stimuli themselves, and this context of humor presentation has a variety of potential interacting effects on humor appreciation (Forabosco, 1991). The specific context effect of a certain series is a function of the salience of a particular characteristic that the humor stimuli have in common (Forabosco, 1991). Specifying "seriality", "the quality which characterizes a sequence of related jokes (Forabosco, 1991, p. 4)", is a focal definition in the assessment of context effects. According to this criterion, a series of humor stimuli range from theoretically identical to conceptually diverse, and the particular nature of this relationship has specific effects on humor appreciation. Several "Gestalt-like" sequence rules are proposed to define how the context of 
humor presentation affects humor appreciation: "jokes show a tendency to be rated more highly if presented in close succession; a more effective order is from less incongruous to more incongruous jokes; and, a structural link between the jokes determines a preferable order (Forabosco, 1991, p. 29.)" The final conclusion was inferred, not assessed, by Forabosco (1991), and therefore remains an empirical question.

Particular context effect predictions are operationalized by manipulating the salience of a specific aspect of the humor stimuli through the priming of that characteristic. Johnston and Dark (1986) define priming as a basis for top down control of selective processing. The prime stimulus affects the processing of the subsequent test stimulus. Enhancing the salience of a common aspect of the stimuli should increase the appreciation for like stimuli (Forabosco, 1991). By means of this empirical procedure, any element that a series of humorous stimuli share is theoretically amenable to enhanced salience through priming. Isolating a particular aspect of the stimuli and increasing the salience of that characteristic suggests what effect that feature of the stimuli has on humor appreciation.

Several researchers have addressed context effects and content salience in humor appreciation (Derks \& Arora, 1993; Forabosco, 1991; and, Goldstein et al., 1972). The context effect of content salience on humor appreciation is a theoretical alternative to the Freudian prediction that humor appreciation is a drive dependent phenomenon. In contrast to the context prediction of the cognitive model, the Freudian view suggests that sexual or aggressive content is the primary element in humor appreciation because of its affect on motivation. In particular, this model assumes that normally socially unacceptable subjects like sex and aggression are natural drives that need to be released, and humor is a permissible outlet for expressing these drives (Derks \& Arora, 1993). Thus, a principle of drive reduction underlies 
humor appreciation in the Freudian model. The cognitive model rejects the notion of drive reduction and predicts that humor appreciation is dependent upon the series effects of both the salience of any humor content and the salience of structural elements of humor such as incongruity and complexity.

Goldstein et al. (1972) suggest that if specific content salience is key to humor appreciation, then in contrast to the Freudian prediction, any type of content appreciation can be enhanced by priming the salience of that theme. Through the priming of a particular humor content, a cognitive set is established for a particular theme, and this priming lowers the threshold for the enjoyment of subsequent humor stimuli of the same theme (Goldstein et al., 1972). In this "salience hypothesis" the establishment of a facilitating cognitive set is positively related to both humor appreciation and comprehension (Goldstein et al., 1972).

Partly due to occasional failures of replications of Goldstein et al. (1972), the content salience effect is thought to be one element of the more general "context effect hypothesis" which predicts that the humor response is a function of the particular construction of a sequence of jokes or cartoons (Forabosco, 1991). In the "context effect hypothesis" the salience of various elements that a series of jokes and/or cartoons may have in common can influence appreciation scores across a presentation of stimuli. Contrary to the Freudian prediction, Derks and Arora (1993) report that the appreciation of sexual humor is less dependent upon specific content of each joke and more dependent upon both the total context of the content of surrounding jokes and the gender of the humor recipient. These results of priming humor content suggest appreciation for jokes within a series depends upon the configuration of the series. The appreciation of any particular joke in a multiple presentation of humor stimuli is not only influenced by the serial relation of that joke with other jokes in a sequence, but also 
affected by the specific nature of the characteristic relationship, similarity or diversity, between the jokes in the series. Both Forabosco (1991) and Derks and Arora (1993) suggest that potential serial effects of humor structure presentation on appreciation for humor is an area requiring further empirical evaluation. The purpose of the experiments was to test the "context effect hypothesis" as it relates to humor structure. Support for the "context effect hypothesis", in terms of humor structure, may illustrate how serial presentation is relevant to appreciation for INC-RES and NON as independent humor structures.

\section{Correlational study}

The correlational study replicated Ruch's (1992) method as a means of addressing the role of the processing variable tolerance in the appreciation of humor. The general purpose of the first study was not only an attempt to determine if relative preference for humor structure is dependent upon tolerance for ambiguity, an affective avoidance of negatively reinforcing stimuli, as explained by Ruch (1992), but also to ascertain if a preference for humor structure is also influenced by a cognitive tolerance for ambiguity, an intellectual enjoyment derived from playfully thinking about the levels of the incongruous nature of humorous stimuli. The specific purpose of this study was two-fold: an evaluation of Ruch's (1992) conclusions concerning humor appreciation, affective tolerance and structure with an American sample; and an assessment of the "Need for Cognition" scale (Cacioppo \& Petty, 1982) as a predictor of humor appreciation. The "Need for Cognition" (Cacioppo \& Petty, 1982) scale was compared to the "Sensation seeking" scale (Zuckerman, 1979) and a modified version of the "Conservatism" scale (Wilson, 1973) as competing predictors of appreciation for INC-RES and NON humor in a modified version of the 3WD humor scale (Ruch, 1983). Predictions concern the conceptual relationship between tolerance for ambiguity and intellectual enjoyment. Conservatives (Wilson, 1973) may have a low need for cognition (Cacioppo \& Petty, 1982) and, prefer 
INC-RES because it is intellectually straightforward. Sensation seekers (Zuckerman, 1979) may have a high need for cognition (Cacioppo \& Petty, 1982 ) and, enjoy NON because it is cognitively challenging. If Need for Cognition (Cacioppo \& Petty, 1982) significantly predicts preference for INC-RES and NON, then it is a useful predictor of humor structure preference. Although the cognitive processing of humor is influenced by affective tolerance, an independent influence on preference for humor structure may be intellectual enjoyment, or a cognitive tolerance.

Method

\section{Subjects}

Seventy-five subjects from the College of William and Mary Introductory Psychology subject Pool voluntarily participated in the study as a partial class requirement. Eight groups, created by a Latin square method for randomized orders, of the four questionnaire presentations were formed to control for potential order effects. Thirty-six male and thirty-nine female subjects were randomly assigned to one of the eight groups. Each group consisted of eight to ten subjects with approximately equal numbers of male and female participants represented in each group.

\section{Questionnaires}

The Need for Cognition scale (Cacioppo \& Petty, 1982), the Sensation seeking scale, version VI (Zuckerman, 1979), a modified version of a Conservatism scale (Wilson, 1973), and a modified version of the 3WD humor scale (Ruch, 1983) were employed in the study (appendices). For validity and reliability information and survey norms, see Cacioppo and Petty $(1982,1984 \mathrm{~b})$ and Osberg (1987) for the Need for Cognition scale; Zuckerman (1979) for the sensation seeking scale; Wilson (1973) for the Conservatism scale; and Ruch and Hehl (1986a, 1986b) and Ruch $(1988,1991)$ for the 3WD humor scale. 
Sensation Seeking Scale (Zuckerman, 1979)

The sensation seeking scale consists of four subscales: thrill and adventure seeking (TAS); disinhibition (DIS); experience seeking (ES); and boredom susceptibility (BS). In Ruch (1988) the total score is used as the predictor of humor structure preference, consequently the total scale was used in this study. Questionnaire modifications 3WD Humor Scale (Ruch, 1983)

Ruch (1983) includes three structural categories in the original 3WD scale: incongruity-resolution, nonsense and sex. The sex jokes and cartoons were removed from the present version of the 3WD scale. This alteration is not expected to affect the validity or reliability of the scale (Ruch, personal communication with Derks, 1991). The sex items were removed for several reasons.

First, the conservatism scale (Wilson, 1973) loads on the tendermindedness quadrant of the personality space (Ruch \& Hehl, 1986b), and the sex jokes load on the toughmindedness quadrant (Ruch \& Hehl, 1986b). Since the sex jokes are structurally both incongruity resolution and nonsense removing them might have rendered the wilson (1973) scale a better predictor of the non-sex incongruity-resolution jokes and cartoons because, the Wilson (1973) scale emphasizes tendermindedness.

Second, it is possible that because the sex jokes and cartoons involve highly salient content stimuli, they may act as a context variable in subjects' general ratings of all of the jokes and cartoons in funniness and aversiveness (i.e., tenderminded subjects may rate all jokes less funny and more aversive as a residual effect of the sex jokes, and toughminded subjects may rate all jokes as more funny and less aversive as a residual effect of the sex jokes). Finally, Ruch (1992) suggests that the 3WD humor scale (1983) overemphasizes the incongruity-resolution structure with a 
disproportional number of incongruity-resolution jokes and cartoons. In an attempt to ameliorate the emphasis on the incongruity-resolution structure an even number of each structure, 18 incongruity-resolution and 18 nonsense, were included in the modified version of the scale. Conservatism scale (Wilson, 1973)

The Wilson conservatism scale (1973) was designed to assess European subjects; therefore some items are not clearly comprehensible to an average American sample. Several items were changed (i.e., licensing laws to $A B C$ laws) in order to ensure that they may be semantically clear. An attempt was made to ensure that specific meanings and connotations of items were maintained.

\section{Procedure}

All subjects were scheduled to appear and arrived for participation separately. Each subject was tested individually and privately in one of ten small rooms reserved for the study. No other person was present when each subject responded to the surveys. Ruch (1992) indicates that standardized testing conditions are crucial in humor studies because situational factors (i.e., other subjects laughing; experimenter's presence) can influence subjects' responses. Upon arrival each subject was given a questionnaire package with written instructions and an anonymous consent form attached. The rating scales were explained in the instructions as $0=$ not funny or not aversive ranging to $6=$ very funny or very aversive. The written instructions indicated that the subject was participating in four unrelated studies, and that he or she should answer all questions honestly without prolonged thought on any one item, and that once a section was completed that it was not to be returned to for any modification. Each subject was instructed to read the instructions, and either ask questions, or proceed with the consent form and surveys. No subjects asked any questions before proceeding however, during testing several subjects did request a definition for aversiveness. Subjects were also instructed to 
detach the instruction sheet and consent form from the rest of the booklet before turning the booklet in so that his or her anonymity would be maintained. All subjects followed these instructions. Subjects were told that participation should take about one half-hour, but no time limit was specified. Subjects took from 20 minutes to 35 minutes to complete questionnaires and cartoon ratings. After each subject finished he or she was asked about his or her impression of the studies purpose, and then de-briefed. No subject indicated that he or she had guessed the purpose of the study.

\section{Results}

The personality questionnaire responses were used as multiple predictors of both the total humor scores and the subtotal humor scores for the individual structures. Each of the total humor scores, funniness and aversiveness, was divided into an INC-RES and a NON subscore for each measure. A difference score between the INC-RES and the NON subscores was used as a relative preference score in the multiple regression and correlational analyses. The total scores were also analyzed by multiple regression and simple correlations. In addition, the two humor subscores were used in two independent $t$-tests, funniness and aversiveness, which assessed the sample wide structural preference. Order effects for questionnaire and cartoon presentation were assessed by One Way ANOVA. Due to non-significant multiple regression results, only theoretically relevant correlations and t-tests are discussed.

Several nonsignificant correlations are found to be in the predicted direction, and several results are both significant and contrary to Ruch's (1992) theoretical predictions (Table 1).. The correlations between Conservatism (Wilson, 1973) and humor categories and Sensation Seeking (Zuckerman, 1979) and humor categories are found in the predicted direction for: the funniness of both INC-RES and NON for Conservatism (Wilson, 1973); the aversiveness of NON 
Table 1

Correlation Matrix of Nonsense and Incongruity Resolution Jokes and

Cartoons for Funniness and Aversiveness and Personality Variables.

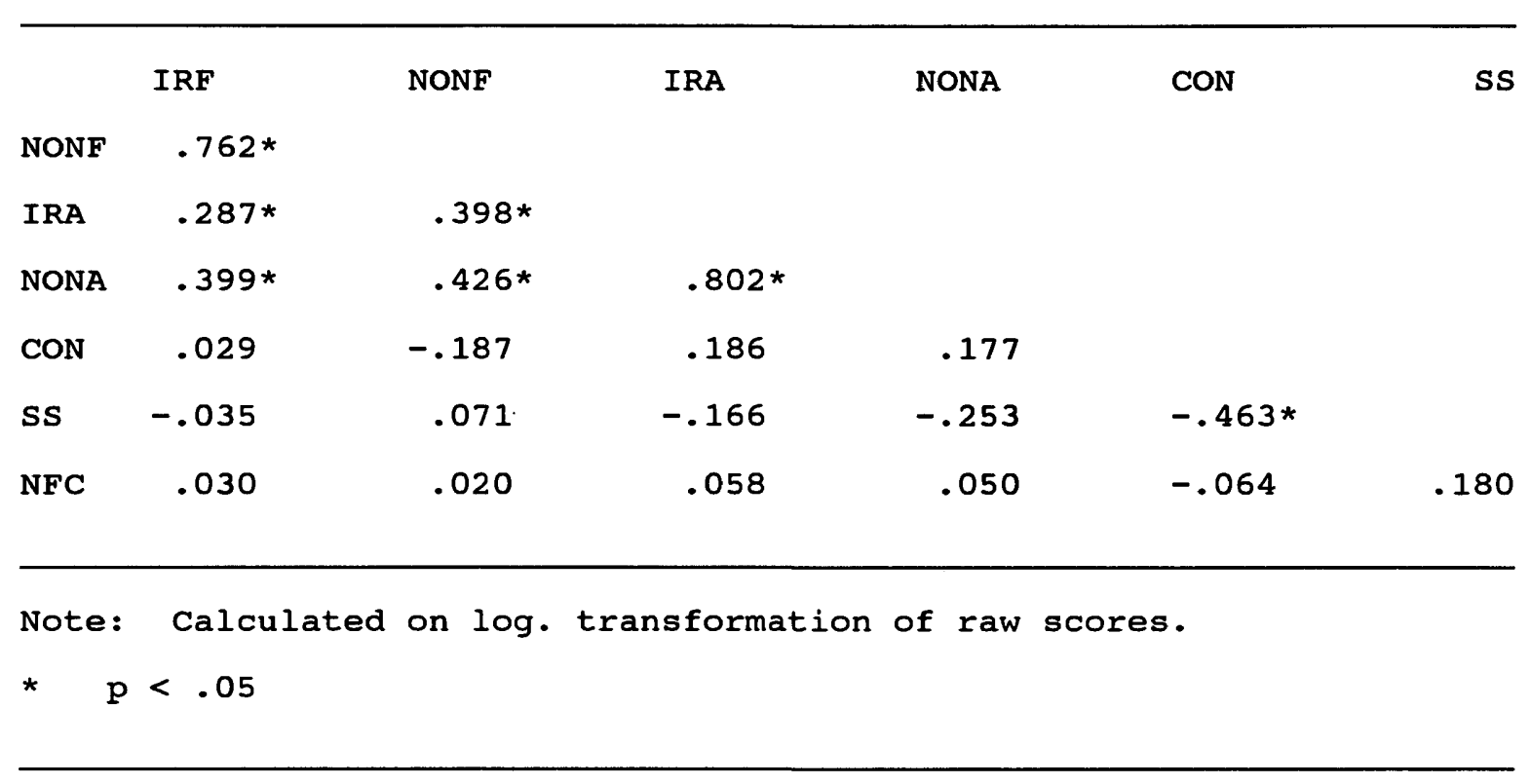


and INC-RES for Sensation seeking (Zuckerman, 1979); and the aversiveness of NON for Conservatism (Wilson, 1973). The correlations between both sensation seeking (Zuckerman, 1979) and Conservatism (Wilson, 1973) and aversiveness of INC-RES are in the reverse of predicted direction. As expected, Sensation Seeking (Zuckerman, 1979) and Conservatism (Wilson, 1973) are significantly and negatively correlated. Although the correlations are not significant, Need for Cognition (Petty \& Cacioppo, 1986) is negatively correlated with Conservatism (Wilson, 1973) and positively correlated with sensation seeking (zuckerman, 1979). In theoretical opposition to Ruch (1991), neither the funniness nor the aversiveness of INC-RES and NON as homogenous and orthogonal categories are supported by the simple correlations. In fact, the funniness and the aversiveness scores for both structures are significantly correlated.

The means, standard deviations, and t-tests for structure preference on the Humor category scores without considering personality variables are presented in Table 2. The means for funniness are similar to those reported by Ruch, Ott and Bariaud (1991) for a French sample (French: Inc-Res 39.37; Non 30.23; Current sample: Inc-Res 37.05; Non 28.32). Standard deviations for the French sample were not reported, therefore it is not known if the differences between the two samples are significant. In these data, the t-test for funniness is significant, but not for aversiveness. For future reference in the subsequent experiments, it is relevant to point out both that INC-RES is rated as funnier than NON and that aversiveness scores do not differ much between INC-RES and NON. Non-significant results from one Way ANOVAS are found for the control for order effects analysis (i.e., on the total humor scale for funniness $\underline{F}(7,65)=0.46, \underline{p}>.85$, indicating that the order of presentation of the cartoons and the questionnaires did not have a significant impact on the results. 
Table 2

Funniness and Aversiveness scores of the Humor Categories.

\begin{tabular}{|c|c|c|c|c|}
\hline Humor Category & Mean & S. D. & $t$ & $\mathrm{p}$ \\
\hline Inc-Res: F & 37.05 & 17.42 & 13.79 & $<.05$ \\
\hline Non: $\mathbf{F}$ & 28.32 & 16.33 & & \\
\hline Inc-Res: A & 13.64 & 12.88 & -1.65 & n.s. \\
\hline Non: $A$ & 15.62 & 11.95 & & \\
\hline
\end{tabular}

Note: t-tests using INC-RES and NON subscores for differences in structure preference on funniness and aversiveness without regard to personality variables. 
Discussion

These data clearly do not replicate Ruch's (1992) prediction that a relative preference for structure is significantly related to individual tolerance for ambiguity as predicted by Conservatism (Wilson, 1973) and Sensation Seeking (Zuckerman, 1979). The correlations between the personality categories and the funniness scores and aversiveness scores indicate that tolerance for ambiguity, defined as a personality variable, did not significantly predict appreciation for a relative structural preference in the current sample. The t-test for aversion was not significant showing that aversiveness scores were assigned fairly randomly across structure (Figure 2). The only conclusion that is both theoretically consistent and significant for structure is based on the funniness t-test where, across personality type, the subjects made some discrimination between the two structures. The mean funniness responses for the individual trials indicate a pattern of INC-RES generally receiving higher ratings for funniness than NON (Figure 1). The correlational aversion results for INC-RES are both in the reverse of predicted direction and large enough to warrant attention. This result may suggest certain methodological problems. Regardless of structure, the Conservative found all humor stimuli more aversive and the sensation seeker found all humor stimuli less aversive. Several of the $\mathbf{s} \underline{\underline{s}}$ asked what aversiveness means which suggests two things. First, if some Ss asked, then perhaps there were others who did not know what it meant and failed to inquire. Those $\mathbf{s} \underline{s}$ who asked were told that aversiveness means distasteful in some way. Second, the definition given may have been misleading. This would be relevant if this instruction lead $\mathbf{S}$ s to attend to humor content as the focal dimension, and they used content as a standard instead of reporting their general reaction to the particular humor stimulus (i.e., the structural dimension of pure of resolved incongruity). Ruch et al. (1991) defines the aversiveness score as a measure that reflects a general negative 


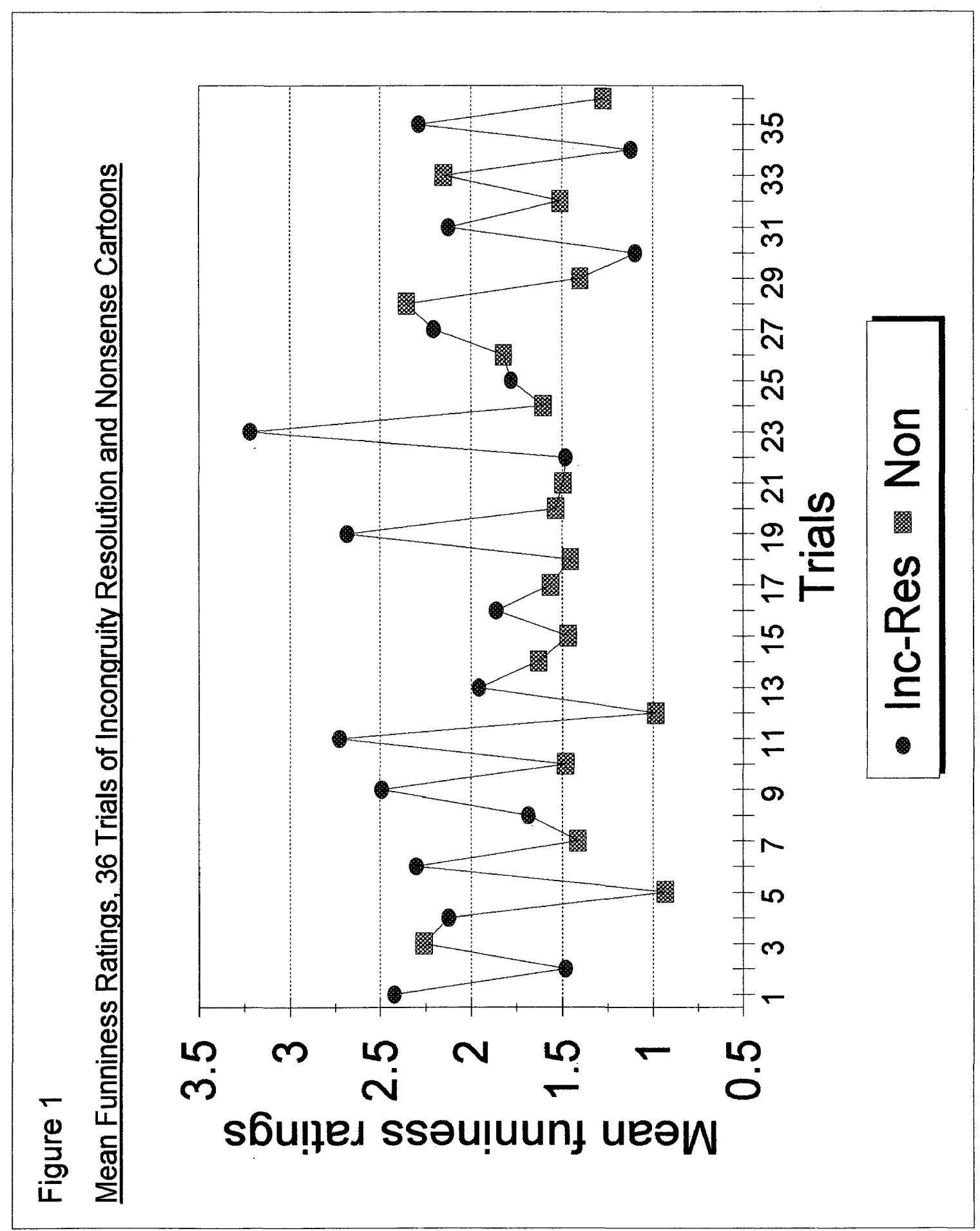




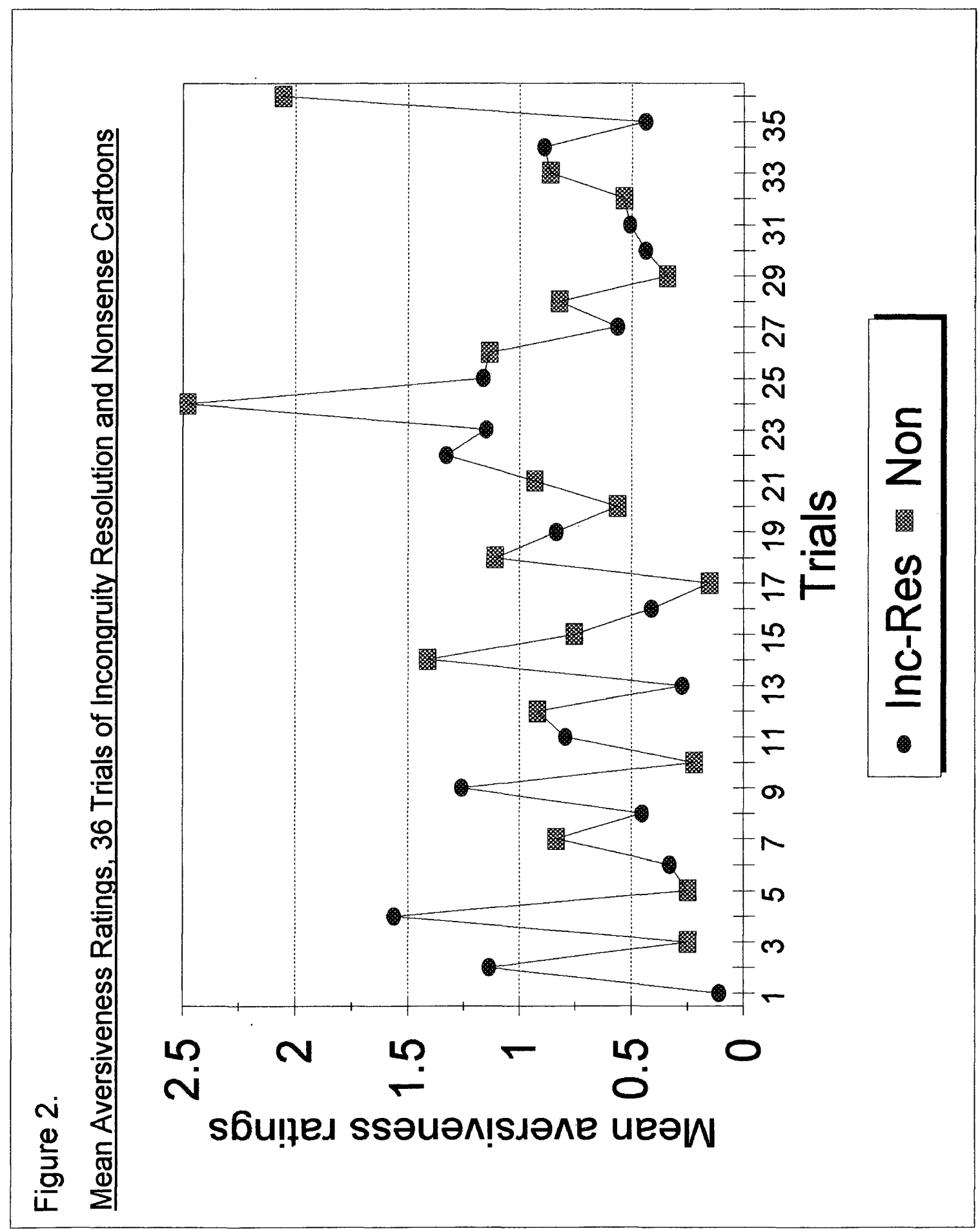


reaction to humor (i.e., irritation, boredom, stupidity, etc.) Because the word aversiveness is the English translation of a German word meaning a general negative reaction, perhaps either the word itself or the stated definition of aversiveness is ambiguous and needs to be. clarified for future assessments of native English speaking subjects. Part of the purpose of the current study was to assess the Need for Cognition (Petty \& Cacioppo, 1986) as a predictor of humor appreciation. The results suggest both that this variable is weakly and positively correlated with sensation seeking (Zuckerman, 1979); slightly and negatively correlated with Conservatism (Wilson, 1973), and that the Need for Cognition (Petty \& Cacioppo, 1986) is not a good discriminator of structural preference. This decision is based on the fact that the signs of the correlations incorporating this variable were neither consistent nor orderly, except in relationships between Need for Cognition (Petty \& Cacioppo, 1986), Conservatism (Wilson, 1973) and sensation seeking (Zuckerman, 1979). As a general conclusion, this supports the idea that information processing in humor appreciation is not greatly related to logical or rational thinking.

However, this empirical finding does not necessarily rule out the theoretical notion that variation in a cognitive tolerance for ambiguity in intellectual stimulation is a factor in humor appreciation. None of the personality variables significantly predicted structural preference thus, the results of the current study cannot rule out the notion of cognitive tolerance as an influence in humor structure appreciation. Although, Need for Cognition (Petty \& Cacioppo, 1986) may not be the appropriate measure, this does not require that cognitive tolerance and humor appreciation are unrelated. Alternatively, this characteristic may be more related to the preferred type of humor (i.e., jokes or puns), or the complexity of humor, or the ability to generate humor. Further theoretical specification and empirical assessment is warranted 
in order to determine any potential relationship between intellectual seeking behavior and humor appreciation or generation.

The significant correlations between ratings for structure are in contrast to the theoretical assumptions not only that the 3WD humor scale (Ruch, 1983) represents a taxonomy of humor with two homogenous structural categories of humor, but also that the ratings of funniness and aversiveness are orthogonal humor scores (Ruch et al., 1991).

"The criterion for establishing a category must be that stimuli within a category are more similar to each other than to stimuli within other categories; the stimuli within a category must be relatively interchangeable...Generally speaking, the criterion for including two jokes in the same category is that they correlate highly with each other across a large set of subjects (Ruch, et al., 1991, p. 393-394)."

The highly significant correlations (Table 1) between INC-RES and NON for both funniness $(r=0.762)$ and aversiveness $(r=0.802)$ shows that these criteria for homogeneity and orthogonality are not met in these current data. These results suggest that for either actual or methodological reasons, relative structural differences were not salient to the subjects. However, the fact that this is a problem for the current sample does not reject the general potential for the homogeneity of categories because the present sample is quite small, and other variables such as cartoon series context effects (Forabosco, 1991) are not taken in account in this correlational study.

These present results indicate that the effect size of the influence of personality on humor appreciation is quite small as an isolated variable. Given that the mean funniness scores are similar in this sample with those Ruch et al. (1991) report for a French sample, the current sample size may have been insufficient to detect significant differences. The fact that the correlations suggest directional trends in line with Ruch's (1992) predictions of the relationship between 
personality and humor appreciation does not warrant rejecting the replication hypotheses outright.

Experiment 1

In the correlational study, INC-RES was generally rated as funnier than NON, and this pattern indicates that those subjects perceived some subtle structural distinction (Figure 1). Several researchers (Rothbart \& Pien, 1977; Ruch, 1992; Shultz, 1972; and Suls, 1972) have reported that jokes and cartoons have structural properties that influence the humor response. In addition, Deckers et al. (1989), Derks and Arora (1993), Forabosco (1991) and Goldstein et al. (1972) demonstrated that the serial presentation of cartoons is a context variable that affects humor appreciation, and therefore the potential effects of cartoon or joke context should be considered when evaluating the results of humor studies. In the "context hypothesis" (Forabosco, 1991) the humor response is a function of both the nature of the relationship between structural elements of the stimuli and the particular juxtaposition of these structural characteristics in a sequence of jokes or cartoons. Empirically demonstrated content context effects indicate that priming content enhances the salience of the particular humor content, and heightened salience is positively related to humor appreciation (Goldstein et al., 1972.). In these content priming studies, humor scores depend upon either the total context of similarity in humor content (Goldstein et al., 1972) or the specific juxtaposition of variation in humorous theme within a series (Derks \& Arora, 1993, and Forabosco, 1991).

Theoretically, context effects for humor structure may also be isolated by manipulating the salience of individual structures through priming (Derks \& Arora, 1993). All humor stimuli were presented in the same order in the correlational study; consequently, context effects of humor content and structural presentation were completely confounded. As a result, subjects in the correlational study may have been 
responding to either humor structure, content or a serially defined interaction of both properties of the humor stimuli. In the two experiments, cartoon content was a controlled variable, and one structure was primed and then on a subsequent trial, a switch to the alternate structure was introduced as means of assessing possible context effect of cartoon structure in series. This method of manipulating structural presentation allowed for a more direct test of the effects of structure on humor appreciation in Ruch's (1983) 3WD humor scale.

Deckers et al. (1989) and Goldstein et al. (1972) have defined several effects that salience may have on appreciation for humor over a series of presentations. In the humor response "interruption of organized processing occurs in a joke when the incongruous punchline is heard or in a cartoon when the incongruous element is perceived (Deckers et al., 1989)". As a result of this process, arousal may accumulate and increase funniness ratings, and this enhanced sensitization positively affects amusement over a series of cartoons (Deckers et al., 1989). However, counteracting this sensitization effect is a habituation process where sensitization reaches a saturation point and appreciation scores begin to decrease (Deckers, et al., 1989). In this "dual process" theory, stimulus sensitization and response habituation are general responses that may apply to various dimensions of humor stimuli (Deckers, et al., 1989). The "salience hypothesis" (Goldstein, et al., 1972) also predicts that in a series of similarly defined cartoons, the increasing salience of the target characteristic of the humor stimuli will increase the possibility of perception of the incongruous aspect, thereby increasing funniness ratings over a series. Each view defines the necessary conditions for an increase in funniness ratings, but only the "dual process theory" explicitly predicts a decrease in appreciation in terms of habituation. 
Content and structure are both necessary but independent features of a joke or cartoon (Ruch, 1992). Derks and Arora (1993) distinguish between the roles of content and structure in the humor stimulus. Content is a readily salient element of humor that can be manipulated by repeated presentation of a particular theme throughout a series (Goldstein et al., 1972), or altering the presentation of a theme in a series of jokes or cartoons (Derks \& Arora, 1993). Humor structure, the degree of incongruity and resolution, is not a variable alterable by changing theme because it is the method of presentation of incongruity. structure is the particular construction of the incongruity of the unique theme. Because a subject can be sensitized to different themes through manipulating the salience of the given content of humor, Derks and Arora (1993) predict not only that priming structure may also heighten the salience of structure, but also that this increased salience may alter appreciation for the particular humor structure. The purpose of the two current experiments was to assess the effects of priming and juxtaposition of structural type in a series of cartoons on the appreciation of the two humor structures, INC-RES and NON humor. If appreciation for INC-RES and NON may be changed by the priming of and the juxtaposition of structure in serial presentation, then this would demonstrate that structure is a salient feature of the humor stimulus that affects appreciation for humor.

Although not evident in the currently reported correlational study, a relative preference for the structure of incongruity has been shown to be, at least in part, related to a trait-dependent tolerance for ambiguity (Ruch, 1992). In this relative "trait" hypothesis, the Conservative finds only INC-RES amusing, but the sensation seeker perceives both NON and INC-RES as humorous. The priming manipulations in the current experiments test potential context-dependent preference due to manipulating humor structure presentation as a competing hypothesis for the "trait" prediction. In this cognitive "state" 
hypothesis, humor appreciation is dependent upon the salience of the type of incongruity, pure or resolved instead of a relative tolerance for ambiguity as defined by Ruch (1992).

Priming may either facilitate or inhibit the processing of subsequent humor stimuli depending upon the nature of the serial relationship between the prime and test stimuli. Similarity between the prime and test stimuli should enhance appreciation for the test stimulus by facilitating processing, and distinctiveness between the prime and test stimulus should diminish appreciation for the test stimulus by interfering with processing. Due to the preference for INC-RES over NON in the correlational study, these effects may also be moderated by the difference between the individual structures degree of resolution of incongruity. Two structural context "state" hypotheses and the relative tolerance for ambiguity "trait" hypothesis (Ruch, 1992) were tested in Experiment 1 .

Hypothesis 1: If appreciation for structure may be enhanced by repeated exposure of a single structural type, then the scores should progressively increase over the series, and the fourth cartoon in the same prime groups (four cartoons either INC-RES or NON) should be rated as both significantly funnier and significantly less aversive from those cartoons that primed it because of facilitation of processing due to a heightened sensitization to structure.

Hypothesis 2: If structure is not only a salient feature of the humor stimuli, but also influential in preference, then the fourth cartoon ("switch structure") in the different prime groups (INC-RES "switch to" NON or NON "switch to" INC-RES) should be rated both significantly less funny and more aversive from those that cartoons primed it because of interference. If INC-RES and NON are rated according to the pattern obtained in the correlational study and priming does not alter this preference pattern, then INC-RES following NON should be found significantly funnier than the NON priming cartoons, and 
NON following INC-RES should be rated as significantly less funny than the INC-RES priming cartoons.

Hypothesis 3: If personality based tolerance for ambiguity, as measured by the sensation seeking scale (Zuckerman, 1979) and the Conservatism scale (Wilson, 1973), is influential in relative humor appreciation, then relative scores on NON and INC-RES should be significantly predicted by scores on these personality variables. Relatively, NON should be both funnier and less aversive for the Sensation Seeker (Zuckerman, 1979) and INC-RES should be both funnier and less aversive for the Conservative (Wilson, 1973). Accordingly, each personality type should show greater priming for humor structure relative to predicted differences in tolerance for ambiguity.

Method

Subjects

A total of 146 William and Mary undergraduates, 51 female and 95 male, voluntarily participated in the first experiment as partial fulfillment of their required research participation for Introductory Psychology classes. There were two types of prime groups which were further defined by structure: two control groups (single structure presentation) and two experimental groups (primed with one structure and switched to the second structure on the final trial). The NoN only control group ( $N=36$ ) included 12 female and 24 male Sg; the INC-RES only control group ( $\mathrm{N}=36$ ) consisted of 13 female and 23 male $\mathrm{S}$; $;$ the NON switch to INC-RES experimental group $(N=37)$ was made up of 13 female and 24 male Ss; and the INC-RES switch to NON experimental group $(N=37)$ was comprised of 13 female and 24 male S.

As control for order effects of both particular cartoons and cartoon content, each of the four tested groups was subdivided into four small groups, four types of combinations of each cartoon presentation group, with each group constructed through Latin square randomized orders of cartoon presentations. These controls yielded a total of 16 
subgroups. Across groups, no cartoon was in the same position for more than one grouping, and no cartoon was followed by the same cartoon in more than one grouping. Content and cartoon randomization was critical for inferences concerning the context effects of structural presentation.

\section{Humor scale}

A total of eight cartoons, four nonsense cartoons and four incongruity resolutions cartoons were selected from the 3WD (forms $A$ and B) humor scale (Ruch, 1983) as the best examples of INC-RES and NON based on correlational analyses from experiment 1 (see appendices for cartoons). The cartoons selected had individual correlations for funniness and aversiveness in the appropriate direction for Ruch's (1992) predictions. Specifically, the Ss who scored high on sensation Seeking (Zuckerman, 1979) rated the NON cartoons as funny and non-

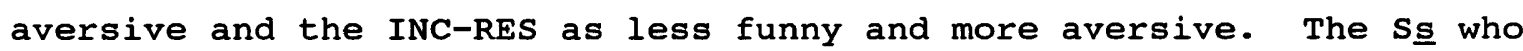
scored high on Conservatism (Wilson, 1973) rated the NON jokes as less funny and more aversive and the INC-RES as more funny and less aversive. Each cartoon was on a separate page with spaces indicated for reporting funniness and aversiveness scores. Consistent with the correlational study, funniness was rated as $0=$ not at all funny to $6=$ very funny; and, aversiveness was rated as $0=$ not at all aversive to 6 = very aversive. In the correlational study, there were no order effects for questionnaire presentation therefore, the sensation seeking Scale (Zuckerman, 1979) and the same modified Conservatism Scale (Wilson, 1973) used in the correlational study were presented after the series of cartoons.

Procedure

Subjects were tested as a large group in an auditorium. Upon arrival the $\mathbf{S}$ s were told that they would be asked both to rate several cartoons for funniness and aversiveness, and to respond to two questionnaires. The rating scales were then explained to the subjects. 
In correlational study 1 several $\mathbf{S} \underline{\underline{s}}$ indicated that they did not know what aversive meant therefore, in this experiment the experimenter also indicated that aversiveness is a general negative response indicating that a cartoon is distasteful for some reason. It was also stressed that a cartoon could differ on the two scales, and not funny did not necessarily mean that it was aversive. The $\mathrm{S} \underline{\mathrm{s}}$ were asked: if they had questions; to read and sign the consent form (anonymous and no deception) if they wished to participate; finally, to begin the task. Upon completion, the Ss were thanked, de-briefed, and solicited for questions.

\section{Results}

simple correlations between the funniness and aversiveness sum scores for each structure were obtained in order to determine if the distinction between the structures evident in the main effects for prime in the MANOVAS were independent ratings. For INC-RES, the funniness and aversiveness sum scores were not significantly correlated (Table 5) indicating that subjects discriminated between the two ratings for INCRES. However, for NON sumscores these ratings were significantly correlated (Table 4) suggesting that subjects found funny NON also aversive. These results suggest that although subjects may have distinguished between structures, they did not consistently apply to the ratings of funniness and aversiveness across structures.

Funniness and aversiveness ratings were analyzed by separate 2 (prime structure) $\times 2$ (switch condition) $\times 4$ (trials) MANOVAs. In the funniness analysis (Figure 3), a significant between groups main effect for prime structure, $\underline{F}(1,145)=5.11, \underline{p}<.05$, shows that scores for NoN were significantly lower than scores for INC-RES. The predicted between groups interaction for prime structure and switch structure failed to reach significance, $\underline{F}(1,145)=1.09, \underline{p}=.29$, which suggests that funniness scores did not differ significantly as a function of prime structure and switch structure juxtaposition. The hypothesized within 


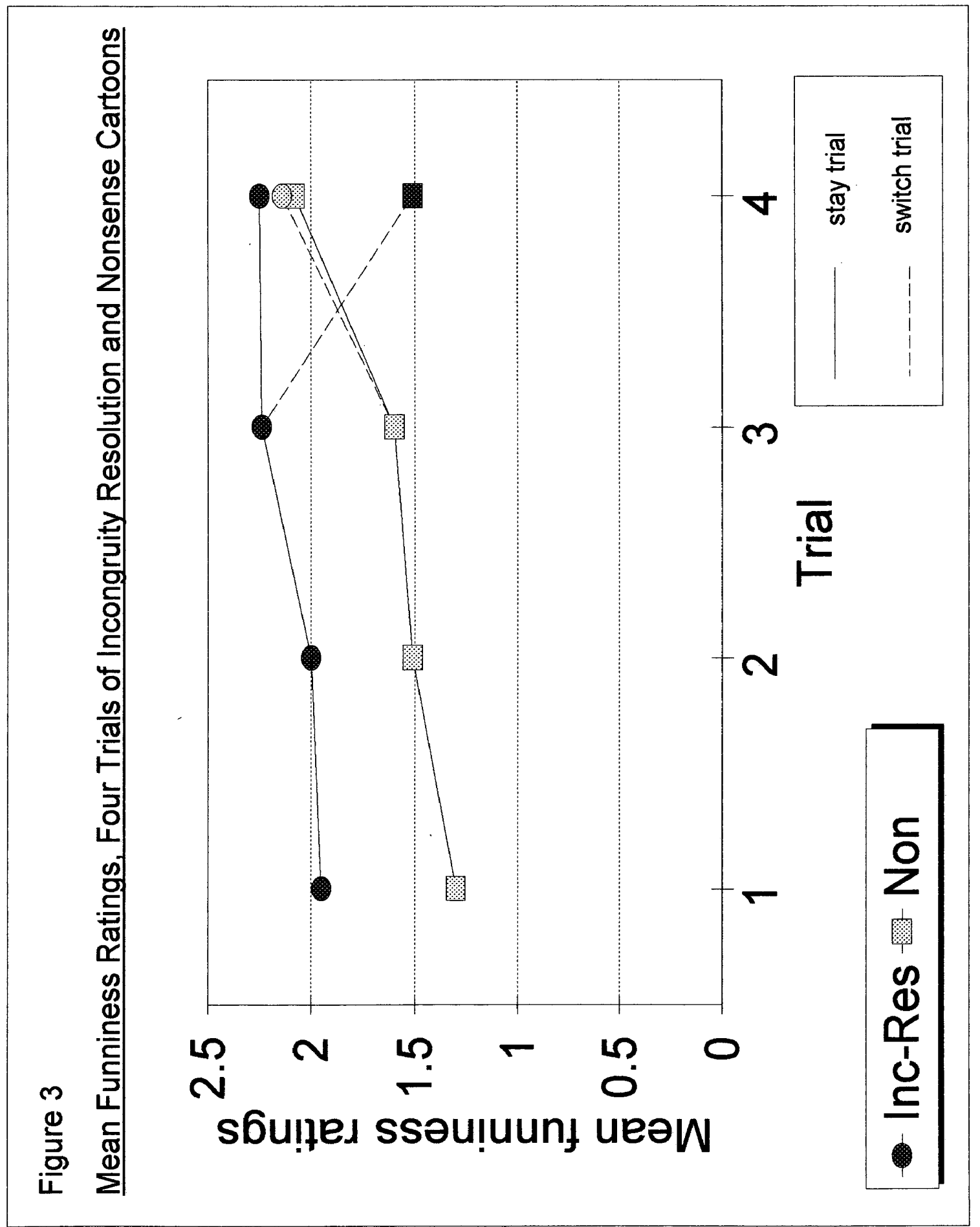


subject interaction between prime structure, switch manipulation and trial was not significant, $\underline{\mathbf{F}}(3,143)=1.02, \underline{p}=.32$ (Pillais approximate F), suggesting that priming combined with the switch treatment did not have a significant effect on funniness scores across the series. A significant within groups interaction for prime structure and funniness trial, $\underline{F}(3,143)=2.63, \underline{p}<.05$ (Pillais approximate $F$ ), shows that scores differed by structure across trials.

Since the MANOVA was not a direct test of the prime structure by switch structure hypothesis, a planned comparison difference score ANOVA was used to test the effects of priming with one structure on the first three trial and then switching to the other structure on the fourth trial. The difference score was the rating of the fourth cartoon minus the mean of the first three cartoon ratings. This analysis indicates a significant prime by structure interaction at the fourth trial, $\underline{F}(1,145)$ $=7.55, \underline{p}<.008$. Subjects' ratings of the fourth cartoon differed significantly depending upon whether they were primed with the same structure on the first three trials or whether they were primed with the alternate structure on the first three trials (NON/NON, $m=.65, \mathrm{n}=36$; INC-RES/INC-RES, $m=.13 ;$ INC-RES/NON, $m=-.46, \mathrm{n}=37 ;$ NON/INC-RES, $m$ $=.63, \mathrm{n}=37$ ). Subjects showed a significantly lower appreciation for NON on the fourth trial if they were primed with INC-RES than if they were primed with NON, and subjects found INC-RES on the fourth trial significantly funnier after priming with NON than after priming with INC-RES. A contrast effect for appreciation of structure may explain the ANOVA interaction. It appears that the less congruous structure, NON primes appreciation for the more congruous structure, INC-RES; but, the more congruous structure, INC-RES interferes with appreciation for the less congruous structure, NON.

In the aversiveness analysis (Figure 4), a significant between groups main effect for prime structure, $\underline{F}(1,145)=12.19, \underline{p}<.01$, signifies that scores for NON were significantly less aversive than 


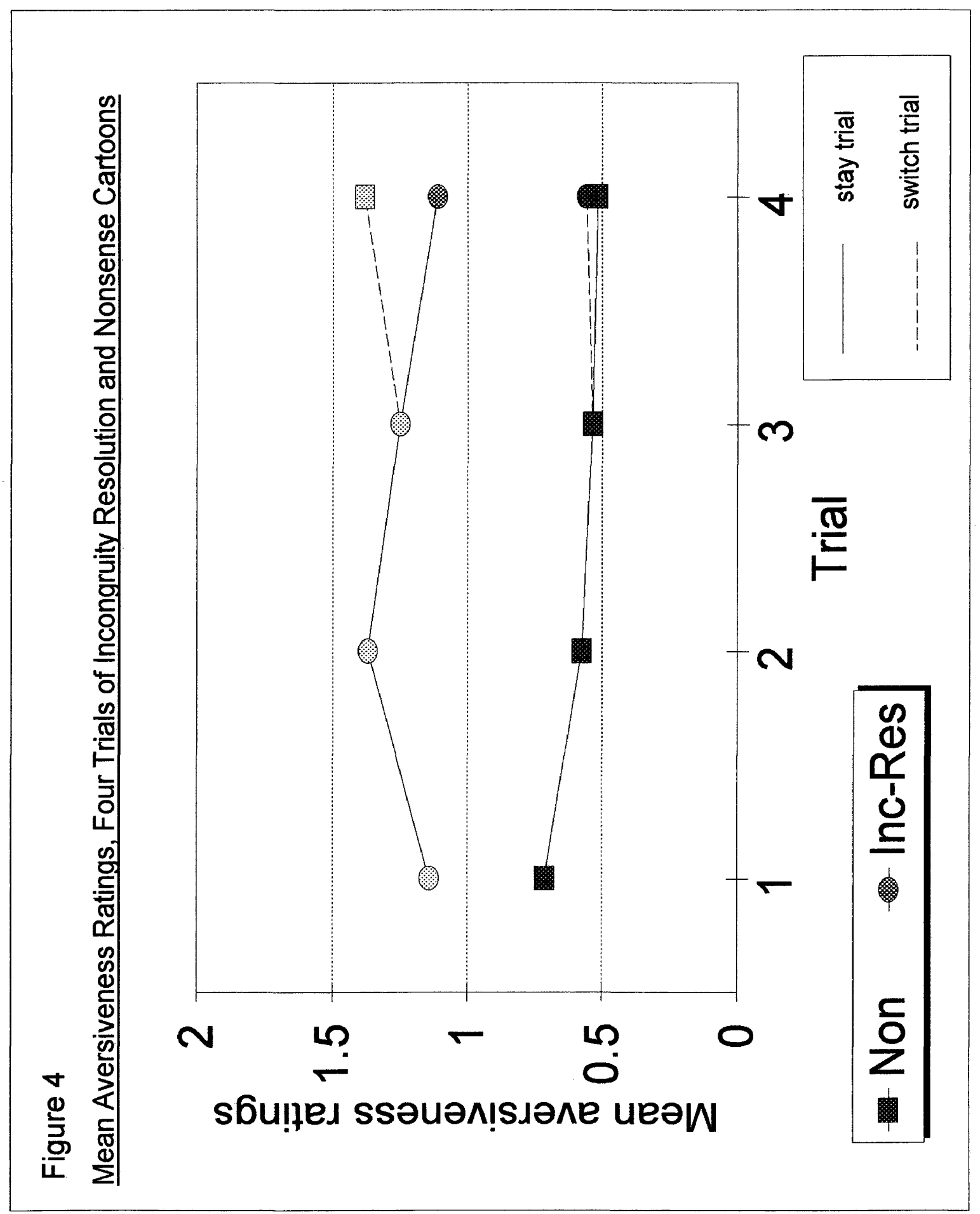


scores on INC-RES. The between groups prime structure and switch interaction also failed to reach significance for aversiveness, $\underline{F}(1,145)$ $=.92, \underline{p}=.33$, demonstrating that the priming and switch manipulations were not significantly influential in these data. The within subject interaction between prime structure and trial failed to reach significance, $\underline{F}(3,140)=1.96, \underline{p}=.12$ (Pillais approximate $F$ ), suggesting that aversiveness scores did not differ significantly by prime structure and trial. Finally, a significant within subject interaction between prime structure, switch manipulation and trial was obtained for aversiveness scores, $\underline{F}(3,140)=3.15, \underline{p}<.05$ (Pillais approximate F), showing that scores differed across the series depending upon prime structure and switch manipulation.

Simple correlations between the predictors, scores on the Sensation Seeking scale (Zuckerman, 1979) and the Conservatism scale (Wilson, 1973), difference scores and sumscores were calculated. A difference score, calculated by subtracting the average of the first three scores from the fourth score for the single structure presentation groups, was used as an overall dependent measure of preference due to priming effects. Correlations between the difference scores and the personality variables are presented in Table 3. A sum of the first three cartoons (i.e., all subjects rated either all INC-RES or all NON on the first three cartoons) was used as a dependent measure of preference for the individual structures; consequently, there was a NON sum score and an INC-RES sum score. Correlations between the sumscores and the personality variables are presented in Table 4 for NON, and Table 5 for INC-RES.

The difference score analysis of the relationship between priming effects on funniness and aversiveness for the final cartoon and the personality variables indicates that funniness scores but not aversiveness scores significantly correlate with the personality variables. A significant positive relationship between the funniness 
Table 3

Correlation Matrix of Difference Scores for Funniness and Aversiveness and Personality Variables.

\begin{tabular}{llll}
\hline & ADSCR & CON & SS \\
FDSCR & $-0.302 * *$ & $0.242 *$ & $-0.223 *$ \\
ADSCR & & 0.018 & 0.1558 \\
CON & & & $-0.456 * *$ \\
\hline
\end{tabular}

Note: across structure, a positive DSCR indicates an increased funniness or aversiveness for the final cartoon; a negative DSCR suggests a decreased funniness or aversiveness for the final cartoon; and, a zero score shows no preference.

$* \mathrm{p}<.05$

$* * \mathrm{p}<.001$ 
difference score and Conservatism (Wilson, 1973) and a significant negative relationship between the funniness difference score and Sensation Seeking (1979) suggests that more Conservative subjects were less likely to have adapted to structure presentation when the single structure was primed than the higher sensation seeking (Zuckerman, 1979) subjects. The significant negative relationship between the aversiveness and funniness difference scores indicates that higher funniness scores are associated with lower aversiveness scores. This suggests that adaptation to structure is related to higher aversion for structure.

The significant relationships between individual structure sumscores and personality variables suggest that funniness for NON but not INC-RES is related to the personality variables, and aversiveness for INC-RES is related to Conservatism (Wilson, 1973) only. Significant correlations (Table 4) indicate both that higher sumscores for NON are associated with higher sensation seeking (Zuckerman, 1979) and lower Conservatism (Wilson, 1973), and that higher sumscores for funniness of NON are also related to higher sumscores for aversiveness of NON. significant correlations (Table 5) show that higher aversiveness for INC-RES is associated with higher Conservatism (Wilson, 1973), and although nonsignificant, higher scores for INC-RES are associated with both higher Conservatism (Wilson, 1973) and lower Sensation seeking (Zuckerman, 1979). The significant results suggest that appreciation for NON is related to a "trait" based tolerance for ambiguity as predicted by Ruch (1992). The nonsignificant but suggestive relationships between Conservatism (Wilson, 1973) and Sensation Seeking (Zuckerman, 1979) are congruent with Ruch's (1992) conclusion that subjects with a high tolerance for ambiguity find INC-RES less funny than subjects with a low tolerance for ambiguity. These correlations may fail to reach significance because of Ruch's (1992) prediction that INC-RES and NON are both funny for the subjects with a higher tolerance 
Table 4

Correlation Matrix of Funniness and Aversiveness Sumscores for NoN and the Personality Variables.

$\begin{array}{llll} & \text { ASUM } & \text { CON } & \text { SS } \\ \text { FSUM } & 0.313 * * & -0.287 * & 0.317 * * \\ \text { ASUM } & & 0.082 & 0.025 \\ \text { CON } & & & -0.465 * *\end{array}$

* $p<.05$

$* * \mathrm{p}<.01$ 
Table 5

Correlation Matrix of Funniness and Aversiveness Sumscores for INC-RES and the Personality Variables.

\begin{tabular}{llll}
\hline & ASUM & CON & SS \\
FSUM & 0.079 & 0.211 & -0.107 \\
ASUM & & $0.239 *$ & -0.073 \\
CON & & & $-0.590 * *$ \\
\hline
\end{tabular}

* $\mathrm{p}<.05$

$* * \mathrm{p}<.001$ 
for ambiguity. Taking the correlational results together, it is suggested that, in general, Conservatives (Wilson, 1973) may find humor less funny and more aversive than sensation Seekers (Zuckerman, 1979). Multiple regression analyses were used to predict preference for humor structure sumscores from the scores on the sensation seeking scale (Zuckerman, 1979) and the Conservatism scale (wilson, 1973). The regression analyses were significant for predicting humor funniness for NON humor only. R square for the full equation $=0.129$. For the predictors Sensation Seeking (Zuckerman, 1979): $\mathrm{b}=.285 ; \underline{t}=2.488, \mathrm{p}<$ .05 , Conservatism (Wilson, 1973): $\mathrm{b}=-.246 ; \underline{t}=-1.936, \underline{p}<.05$ are these multiple regression results. Only aversion scores for INC-RES were significantly predicted by the Conservatism (Wilson, 1973): $R$ square $=.057 ; b=.239 ; \underline{t}=2.078, \underline{p}<.05$. These funniness results conform to the relationship between the personality variables and preference for NON humor predicted by Ruch (1992). However, neither the nonsignificant predictions for funniness of INC-RES nor the significant prediction of aversiveness for INC-RES concur with Ruch's (1992) model that personality based relative tolerance for ambiguity as defined by Conservatism (Wilson, 1973) and sensation seeking (1979) significantly predict humor scores for structure.

It is concluded from significant main effects for prime structure in the MANOVAS (Figures 3 and 4 ), the significant planned comparison ANOVA, and the correlational results (Table 3 ) that funniness and aversiveness ratings for the final cartoon in the series are partly a function of cartoon structure and a priming effect that may moderated by personality based tolerance for ambiguity. The significant ANOVA interaction suggests that the structure of the cartoons that precede the switch cartoon had some priming influence on appreciation for the final cartoon. Appreciation scores for INC-RES increase only in contrast to NON, and appreciation scores for NON are enhanced by unique presentation, but they diminishes in comparison to INC-RES. Therefore, 
the interaction between prime structure and switch structure suggests that structure salience had some effect on appreciation scores. There is evidence that INC-RES does not prime NON, but NON does prime INC-RES. Due to randomization of cartoon content, a large amount of error variance existed in these data which suggests that this interaction may reflect a fairly robust effect. The results of the personality predictions indicate that tolerance for ambiguity may be most relevant not only to a general habituation to same structure presentation, but also appreciation for NON and aversiveness for INC-RES. The finding for NON agrees with Ruch's (1992) notion that NON is more stimulating than INC-RES because it is unresolved, and this difference in degree of resolution of incongruity will affect appreciation scores. The relationship between aversiveness for INC-RES suggests that Conservatives (Wilson, 1973) may find all humor more aversive than Sensation Seekers (Zuckerman, 1979) which is also coherent with Ruch (1992) that tolerance for ambiguity is an influential variable in appreciation for humorous incongruity.

\section{Discussion}

Significant main effects for funniness and aversiveness of prime structure; a significant prime by structure interaction on the final cartoon presentation, and obtained relationships between differing tolerance for ambiguity and varying resolution in structure indicate that INC-RES and NON are both salient as independent structures in the 3WD cartoons (Ruch, 1983) currently tested. The obtained difference in funniness between INC-RES and NON both significantly replicates the pattern in the correlational study of INC-RES being rated funnier than NON, and indicates that the content "salience hypothesis" (Goldstein, et al., 1972) requires further elaboration in order to apply to the taxonomy of humor structure as a salient dimension of humor: resolution is salient and readily appreciated; a lack of resolution is also salient but must be primed before it is appreciated; and, not only the absence 
or presence of resolution may interact in appreciation when both structures are presented in series, but also an enhanced salience of structure may account for decreased as well as increased humor ratings. According to the first cognitive "state" hypothesis, repeated exposure to a single structure should increase funniness and decrease aversiveness for that structure. A slight priming effect, an enhanced appreciation, is suggested by MANOVA results in the same prime conditions for each structure (Figure 3 ). This result suggests that funniness for structure is perhaps due to both the "state" context effect of serial presentation, and a moderating effect of tolerance for ambiguity that differentiates a possible susceptibility to habituation for same structure presentation (Table 3 ). These funniness results support Forabosco's (1991) previously untested sequence rule that creating a structural link between cartoons is the preferable order for increasing humor appreciation. Aversiveness ratings, on the other hand, do not differ through priming for either INC-RES or NON which indicates that aversiveness may not be altered by repeated exposure to structural incongruity. The significant relationship between Conservatism (Wilson, 1973) and aversiveness for INC-RES may indicate that the aversiveness ratings are more a function of tolerance for ambiguity than context presentation.

The main effect for structure, INC-RES is funnier than NON, suggests that NON is salient by its lack of a resolution of incongruity. Perhaps the strangeness of NON requires an enhanced sensitization (Deckers, et al., 1989) to its structure through priming in order to be perceived as funny as INC-RES by the final trial. This pattern is congruent with both Ruch's (1992) notion that the uncertain resolution in NON is more arousing than the evident resolution in INC-RES, and the idea that pure incongruity can also lead to a response of fear or perplexity (Morreall, 1989). On the other hand, INC-RES is immediately found funny (Figure 3 ) indicating that INC-RES does not require priming 
in order to be appreciated as funny. According to a "dual process" (Deckers et al., 1989) formulation, sensitization to INC-RES is already primed perhaps through previous experience with INC-RES. INC-RES is the more "socialized" form of humor (many cartoons and most jokes are INCRES; NON is less common and usually in cartoon form). Perhaps subjects respond immediately and positively to INC-RES because the resolved incongruity is congruent with the expectation that even though humor may be initially nonsensical, it ultimately involves a sense of certainty. Morreall (1989) suggests that it is adaptive to determine nature of incongruity, and this is achieved through the resolution of incongruity. The effects of priming NON and INC-RES offer some support for Forabosco's (1991) sequence rule that establishing a structural link is an effective method of presentation for increasing appreciation scores. This is the case for INC-RES because although priming may not be required for immediate appreciation, repeated exposure does enhance funniness for INC-RES.

However, the potentially different requirements for priming sensitization for each structure have distinct implications for the dual process prediction (Deckers et al., 1989) of response habituation within a series of cartoons. Habituation to humor content stimuli may occur between the third and the fifth cartoon (Deckers et al., 1989), and this effect may also be present for humor structure. Habituation may receive tentative support with INC-RES because the funniness ratings begin to flatten from the third to fourth trial (Figure 3). INC-RES seems to be readily appreciated (salient), but because it is more easily accessible, perhaps this also makes it predictable, and therefore rendered less arousing more quickly. Because only four cartoons were presented in this experiment, a control group in Experiment 2 was assessed in terms of the habituation predictions for INC-RES. The effects of priming NON supports the salience hypothesis (Goldstein et al., 1972) and sensitization prediction of the dual process theory (Deckers et al., 
1989). The apparent requirement for priming NON in order to enhance salience and thus appreciation may render NON more durable to habituation effects. However, this possibility was also addressed in the NON control group in Experiment 2 where a longer series of single structure cartoon was presented.

According to the second cognitive "state" hypothesis, switching from one structure to the other on the fourth trial should alter appreciation for the final cartoon depending upon either interfering effects of priming or a preference for INC-RES over NON. The planned comparison ANOVA indicates that appreciation for the final cartoon presentation depends upon both the prime structure and the switch structure (Figure 3). Appreciation for NON is significantly less after priming with INC-RES than if NON is presented alone, and appreciation for INC-RES is primed either by INC-RES or by NON. This significant pattern strongly contradicts Forabosco's (1991) prediction that the more appreciated order is from the less incongruous structure (INC-RES) to the more incongruous presentation (NON). The obtained ANOVA interaction also supports a "salience hypothesis" (Goldstein et al., 1972) as applied to structure. A structural distinction or contrast effect may be perceived by the subjects because they responded significantly differently to the cartoons on the switch trial.

The main effect for prime structure, the significant ANOVA interaction between prime structure and switch structure, and the relationships between the difference scores and the personality variables support the cognitive "state" hypotheses. Taken together, these results suggest that enhancing structural salience through priming and tolerance for ambiguity are relevant in appreciation scores. However, these results do not rule out independent relative personality predictions for structural preference Ruch (1992). If, as Ruch (1992) suggests, the stimulative quality of humor structure, either novel or predictable, is related to a tolerance for uncertainty, then sensation 
Seeking (Zuckerman, 1979) and Conservatism (Wilson, 1973) should predict a relative preference for humor structure. These predictions are supported for funniness of NON and aversiveness of INC-RES in the sumscore correlational analyses (Tables 4 and 5 ). In addition, suggestive correlations indicating that the higher sensation seeking (Zuckerman, 1979) and the lower Conservative (Wilson, 1973) subjects found INC-RES less funny than the lower Sensation Seeking (Zuckerman, 1979 ) and higher Conservative (Wilson, 1973) subjects offers some subtle support for Ruch's (1992) predictions concerning the relationship between tolerance for ambiguity and relative appreciation for pure and resolved incongruity.

A significant multiple regression analysis also indicates that Sensation Seeking (Zuckerman, 1979) and Conservatism (Wilson, 1973) predict funniness scores for NON. The positive relationship between Sensation Seeking (Zuckerman, 1979) and NON and the negative relationship between Conservatism (Wilson, 1973) and NON support Ruch's (1992) conclusions that personality and humor structure appreciation are related. On the other hand, neither sensation seeking (Zuckerman, 1979) nor Conservatism (Wilson, 1973) predict funniness scores for INC-RES. The more arousing NON (Ruch, 1992) not only seems to be more closely related to a tolerance for uncertainty than INC-RES, but also the more predictable INC-RES appears to be more readily appreciated regardless of tolerance for uncertainty. These results offer some support for Ruch's (1992) conclusion that although INC-RES is preferred by both personality types, a relative preference is predictable by tolerance for ambiguity. The "state" and the "trait" hypotheses are therefore compatible for funniness of structure, but significant support for the "state" hypotheses would offer a more complete and more parsimonious explanation of the obtained results. Context effects, personality variables defining tolerance for ambiguity and humor structure are assessed in 
Experiment 2 in order to determine the competitive usefulness of these predictions.

Ruch (1992) suggests that the aversiveness response is a second independent measure of humor appreciation. A significant positive relationship between the funniness and aversiveness scores, in the correlational study, indicate that those subjects rated cartoons similarly for both funniness and aversiveness. This relationship is contrary to Ruch's (1992) contention that funniness and aversiveness are orthogonal scores, but it was also a potential result of an inadequate explanation of the term aversiveness. In the first experiment, the aversiveness measure was defined as representing a negative or distasteful reaction to the cartoon, and it was explained that a cartoon can vary on each rating (i.e., a cartoon can be funny and aversive or not funny and aversive), and the funniness scores and the aversiveness scores were not correlated for INC-RES suggesting that these subjects did discriminate between the two types of responses. However, the two responses were significantly positively correlated for NON suggesting that, as in the correlational study, these subjects rated funny NON as also aversive.

A significant regression analysis and simple correlations indicate that as Conservatism (Wilson, 1973) increases so do aversiveness scores for INC-RES (Table 5). Neither Conservatism (Wilson, 1973) nor Sensation Seeking (Zuckerman, 1979) predict aversion scores for NON, and of course, neither do the simple correlations between the personality variables and aversion scores discriminate aversiveness for NON (Table 4). The more Conservative (Wilson, 1973) subjects may find any humor aversive because any humor entails incongruity. Aversion scores for INC-RES and not NON were predicted by Conservatism, (Wilson, 1973) which is contrary to Ruch's (1992) predictions because it is NON that embodies pure incongruity. However, R-square for this equation is very small which suggests that the absolute effect of Conservatism (Wilson, 1973) 
is minimal. Since Sensation Seeking (Zuckerman, 1979) did not predict aversion for NON, those subjects who seek novelty might be less likely to find uncertainty aversive. The second experiment further tests the relationship between personality and aversion to humor structure.

A relevant difference between the correlational study and the first experiment was the humor stimuli used. The correlational study included all non-sexual INC-RES and NON joke and cartoon items from the 3WD humor scale (Ruch, 1983). The first experiment used only eight cartoons, four NON and four INC-RES specifically selected from the items used in the correlational study because they correlated with the personality predictors in the hypothesized direction. It is interesting that it is the restricted sample of cartoons that shows significant relationships between the personality variables and funniness and aversiveness for humor structure however, the sample size was also twice as large in the first experiment than in the correlational study. It is concluded from the results not only that funniness and aversiveness differ by structure and the manipulation of a switch structure presentation, but also that personality based tolerance for ambiguity may be a moderating variable in appreciation for humor structure presented in primed series.

Experiment 2

Results from the first experiments cognitive "state" hypotheses show how priming, context and the salience of humor structure can alter humor appreciation ratings. As a test of how these results may generalize to appreciation for structure depending upon priming and switch manipulations both in a longer series of cartoons and with additional switch manipulations, an extended design was created. There were several specific goals of the second experiment. First, part of the present design served as a replication test of the interaction between prime structure and switch structure from first experiment. A second goal was to further assess the pertinence of the "salience 
hypothesis" (Goldstein et al., 1972) for describing the effects of structural salience in humor appreciation. A third aim was to evaluate "dual process theory" (Deckers et al., 1989) predictions of sensitization and habituation as relevant to humor structure appreciation. A fourth objective was to further experimentally define and test personality based tolerance for ambiguity [Sensation seeking (Zuckerman, 1979) and Conservatism (Wilson, 1973)] in order to simultaneously test the "state" and "trait" hypotheses. A final goal was to determine the relationship between the "state" hypotheses and the "trait" hypotheses as either complementary or competing explanations for the appreciative response to humor structure.

A longer series of cartoons either omitting or varying the position of the "switch" cartoon was used to test the "state" predictions. The relationship between personality based tolerance for ambiguity was experimentally defined by first categorizing scores on the personality measures, and then using the extreme scores to form personality groups. It was hypothesized that if personality based tolerance for ambiguity. is influential in structural preference, then this would certainly be evident in the extreme cases. For the cognitive "state" hypotheses and personality "trait" hypotheses, the basic issue concerned determining what is the most important factor in funniness and aversiveness scores for cartoon structure when cartoons are both presented in series and content is a controlled variable. According to this rationale, appreciation scores would depend upon either one or an interaction of several of the following: the context created by cartoon serial position; a personality based tolerance for ambiguity; or, the stimulative value of the individual structure.

The general independent "cognitive" hypothesis states: if priming heightens the salience of a humor structure then this enhanced salience creates a context that influences the processing "state" for the appreciation of subsequent presentations of structural stimuli. 
Conceptually analogous to Derks and Arora's (1993) content context effects, the total context effect is predicted to depend upon the particular configuration of the humor stimuli. Variations are operationalized by both the primed structure and where the switch is introduced in the series. Differences in appreciation for structure are predicted depending upon both context and structure. Distinctions are predicted based on the individual nature of each structure. A main effect for prime structure in the first experiment indicates that NoN and INC-RES may be differentially salient. If, as Ruch (1992) suggests, the stimulative value of the structure itself influences appreciation and NON is more arousing than INC-RES because it offers less certainty, then there should be differences in appreciation for the individual structures. The varying stimulative value of the particular structure should alter appreciation in terms of a structural "salience hypothesis". The establishment of a cognitive set for a primed structure should enhance appreciation for like stimuli (Goldstein et al., 1972) and diminish appreciation for different stimuli. In a "dual process" prediction (Deckers et al., 1989) appreciation for like stimuli should be sensitized and enhanced, but also reach a point of diminishing returns where appreciation for like stimuli decreases, and perhaps appreciation for different stimuli should be enhanced because of novelty.

The independent "personality" hypothesis addresses the relationship between humor structure appreciation and "trait" dependent tolerance for ambiguity as measured by the sensation seeking scale (Zuckerman, 1979) and the Conservatism scale (Wilson, 1973). Tolerant subjects should prefer NON and intolerant subjects should appreciate INC-RES, regardless of context presentation. The "complementary" hypothesis predicts that appreciation for humor structure will depend upon and interaction between context, structure and personality. For clarity, hypotheses are stated in a general and independent form. 
Specific directional predictions are discussed in the "Results" and "Discussion" sections.

\section{Cognitive Hypotheses:}

Hypothesis 1: A. If appreciation for humor structure may be enhanced by increasing the salience of structure through repeated exposure of a single structural type, then the rating of the cartoons in the same prime ("stay") group should progressively increase. B. If appreciation for humor structure is enhanced by the priming method, then the ratings of the cartoons that precede the "switch" in the "switch" groups ("stay/switch", "switch/stay", and "switch/switch back") should progressively increase before the "switch".

Hypothesis 2: A. If priming increases the salience of humor structure and this enhanced salience is influential in preference, then the "switch" cartoon (INC-RES "switch to" NON or NON "switch to" INCRES) in the "switch" groups ("switch/switch back", "switch/stay" and "stay/switch") should be rated significantly differently from those cartoons either preceding or following it (depending upon manipulation of structural serial position). B. If the "switch" cartoon is salient by contrast to the prime cartoon(s) and influential in preference, then the "switch/back" cartoon should be rated significantly differently from the "switch" cartoon(s).

Hypothesis 3: If aversiveness ratings may be influenced by increasing the salience of structure through priming, then these ratings should decrease as a result of increased salience of structure, and increase as a result of interference when a "switch" cartoon is introduced.

Personality Hypothesis:

Hypothesis 4: Sensation Seekers (Zuckerman, 1979) and Conservatives (Wilson, 1973) both find INC-RES funny, but only sensation Seekers perceive NON as amusing. In addition, relative to the Conservatives (Wilson, 1973), the Sensation Seekers (Zuckerman, 1979) 
find INC-RES less humorous. Sensation Seekers (Zuckerman, 1979) rate all humor as less aversive than Conservatives (Wilson, 1973). In relative terms however, INC-RES is less aversive for the Conservative (Wilson, 1973) and NON is less aversive for the Sensation Seeker (Zuckerman, 1979). If personality categories that represent different tolerances for ambiguity determine a relative structural preference independently of the priming manipulations, then regardless of structural presentation, funniness and aversiveness score should vary according to structure and personality. Hypotheses are understood relatively according to the aforementioned relationships between the humor structures and the personality variables.

A. For the high sensation seeking (zuckerman, 1979)/low Conservatism (Wilson, 1973) subjects funniness scores for NoN should be significantly greater than funniness scores for INC-RES, and for the low Sensation Seeking (Zuckerman, 1979)/high Conservatism (Wilson, 1973) subjects funniness scores for INC-RES should be significantly greater than funniness scores for NON.

B. For the high Sensation seeking (Zuckerman, 1979)/low Conservatism (Wilson, 1973) subjects aversiveness scores should be significantly lower for NON than aversiveness scores for INC-RES, and for the low Sensation seeking (Zuckerman, 1979)/high Conservatism (Wilson, 1973) subjects aversiveness scores should be significantly lower for INC-RES than aversiveness scores for NON. Method

\section{Subjects}

A total of 340 William and Mary undergraduates voluntarily participated in the second experiment as partial completion of their research requirement for Introductory Psychology classes (Table 6). 287 SI were pre-selected for Mass Testing scores on two personality scales. 53 of the subjects were not defined on the personality variables because they were tested after it was determined that the personality variables 
TABLE 6

Experimental Design

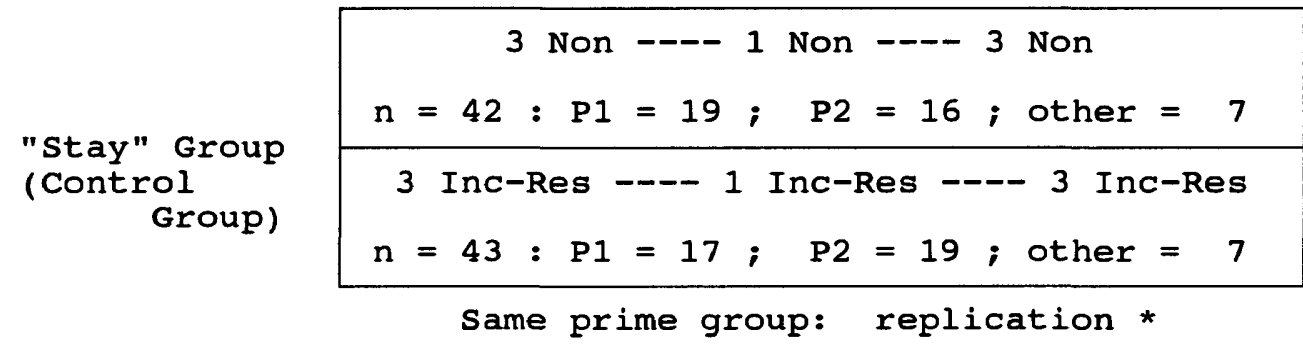

$\mathrm{n}=85$

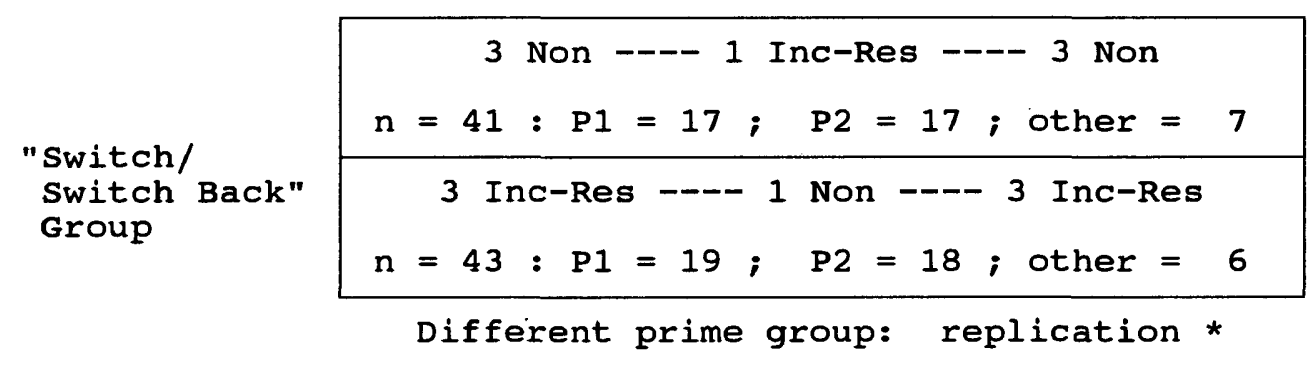

$\mathrm{n}=84$

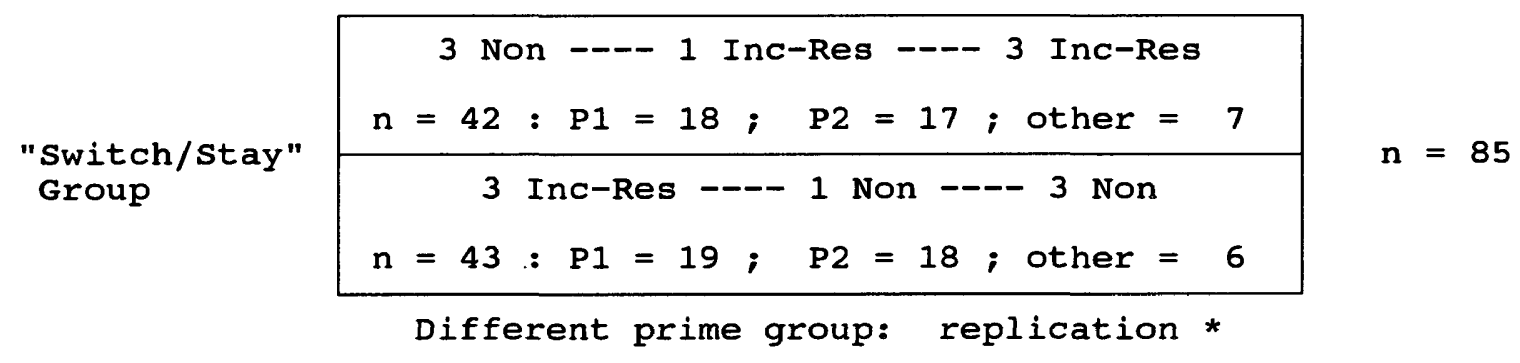

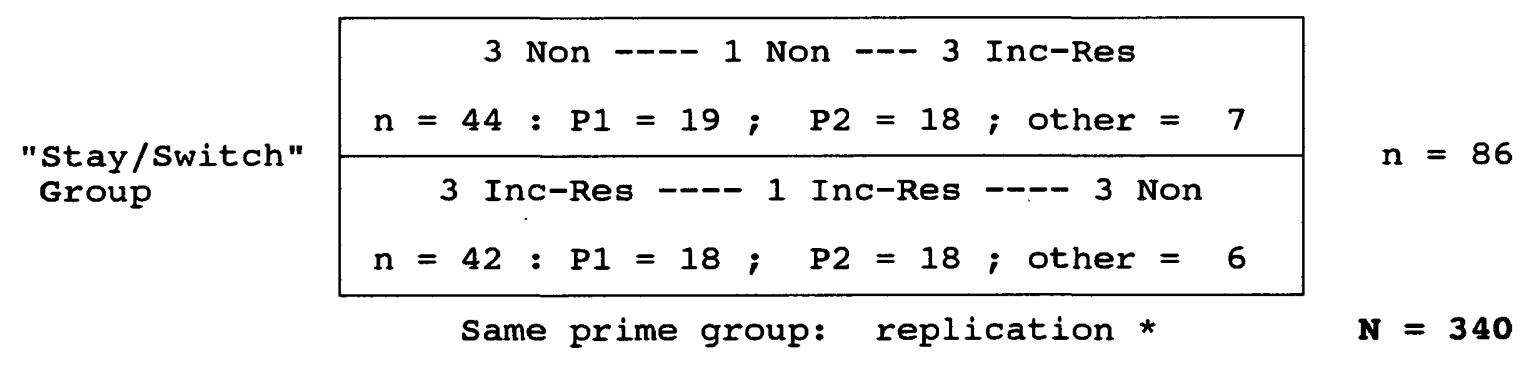

$\mathrm{P1}=$ hiss/locon $; \mathrm{P2}=$ loss/hicon $;$ other $=$ not defined Non $=$ Nonsense structure cartoon Inc-Res = Incongruity Resolution structure cartoon * Groups include first four cartoons only 
were not significant variables in these data. All ss were combined in a single sample because the variance in each separate sample was similar, and they were all tested by the same method under the comparable conditions.

\section{Personality groups:}

One group of $\mathbf{S}$ s was selected for extremely low scores on the Boredom Susceptibility subscale of the sensation seeking scale (Zuckerman, 1979) and extremely high scores on a short version of the

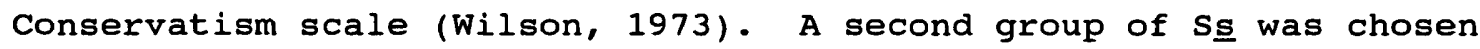
based on extremely high scores on the Boredom susceptibility subscale (Zuckerman, 1979) and extremely low scores on the Conservatism (Wilson, 1973) scale. Pre-selection criteria used to form two extreme personality groups were: hiss/locon: score of 15 or more, out of a possible 20, on sensation seeking subscale (Zuckerman, 1979), score of 3 to 18, out of a possible 64, on Conservatism (Wilson, 1973); and, loss/hicon: score of 10 or 11 on Sensation Seeking subscale (Zuckerman, 1979), score of 30 to 53 on Conservatism (Wilson, 1973).

Prime groups:

The total $340 \mathrm{~S}$ s were assigned in a randomized manner to four prime groups $\left(n^{\prime} s=84\right.$ to 86 ) according to the following criteria (Table 6). These four prime groups were further divided into eight groups (n's $=41$ to 44 ) defined by structure presentation composition. The two personality groups ( $P 1, n=146 ; \mathrm{P} 2, \mathrm{n}=141$ ) were randomized by personality category between 16 groups ( $n^{\prime} s=16$ to 19 ). Each of the four prime groups included two subgroups of each of the personality categories and two of the other (not defined by personality) groups for a total of six groups per prime condition. This design created groups approximately equal in number and equivalently representative of the hypothesized populations.

The first prime group $(\mathrm{n}=85)$ was the "stay" or control group where only one structure was presented across trials. The second 
division of the control group was either 7 NON $(n=42)$ or 7 INC-RES ( $n$ = 43). The second prime group $(n=84)$ was the "switch/switch back" group which was further divided into two groups: 3 NON/ 1 INC-RES/ 3 NON $(n=41)$ and 3 INC-RES/ 1 NON/ 3 INC-RES $(n=43)$. The third prime group ( $n=85)$ was the "switch/stay" group which then formed two groups: 3 NON/ 1 INC-RES/ 3 INC-RES $(n=42$ ) or 3 INC-RES/ 1 NON/ 3 NON ( $n=$ 43). The final prime group $(n=86)$ was the "stay/switch" group which was then divided into either 3 NON/ 1 NON/ 3 INC-RES $(n=44)$ or 3 INCRES/ 1 INC-RES/ 3 NON $(n=42)$. Descriptions of the various cartoon context presentations (i.e., 3 of one structure/ 1 of the same structure/ 3 of the alternate structure) does not mean that there was any break in presentation, These descriptions are merely a convenient way of expressing the priming and switch manipulations in terms of the predictions.

For replication purposes, groupings parallel to Experiment 1 were constructed. These groups were analyzed on the first four cartoons only. For the first three cartoons, the two groupings depended upon which structure was being primed (NON $=180 ;$ INC-RES $=179)$. On the fourth cartoon, the groups were defined depending upon whether a switch structure was introduced at the fourth cartoon. The stay or control group ( $N=180)$ included the "stay" and "stay/switch" groups, and the switch on the fourth cartoon groups ( $N=179)$ combined the "switch/switch back" and "switch/stay" groups (see Table 6).

\section{Humor Scale}

Fourteen cartoons, 7 NON and 7 INC-RES, from the 3WD humor scale (Ruch, 1983) were used in this experiment (Appendices). As in the first experiment, each cartoon was on a separate page with space provided for funniness and aversiveness ratings. The rating scales ranged from 0 (not at all funny or aversive) to 6 (very funny or aversive). Content and cartoon randomization was critical for inferences concerning the context effects of structural presentation. Across groups, no cartoon 
was in the same position for more than one grouping, and no cartoon was followed by the same cartoon in more than one grouping. Thus, in order to control for content effects, 32 randomized orders of cartoon presentations were created through the Latin square technique. For the first four cartoons in each of the four main groups the same cartoons and 16 latin square orders from the first experiment were repeated for replication purposes. For the extended context manipulations (full design), six additional NON and INC-RES cartoons were added, as appropriate per group criterion, also using the Latin square technique for randomizing orders. There are a total of 7 INC-RES cartoons in the 3WD scale, therefore they were all used. Three additional NoN cartoons were selected based on the criterion from experiment 1 (i.e., best predictors according to the personality preference hypothesis).

\section{Procedure}

Subjects were contacted by telephone and scheduled for participation based on individual availability to attend scheduled testing sessions. All subjects were tested in small groups ranging from 10 to 35 participants per session. Upon arrival each subject was asked for his/her name, and it was checked off the list of eligible participants. The Ss was then given a packet from one of eight envelopes representing the context conditions according to the procedure

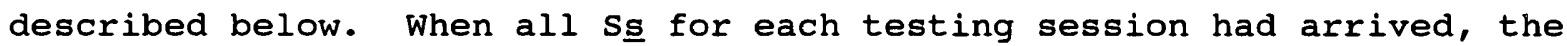
experimenter explained that they would be asked to rate several cartoon for funniness and aversiveness. The rating scales were described and the definition of aversiveness was given. After being told that they may terminate participation at any time without penalty; informed that they may leave if they do not wish to participate; asked to sign the confidential no deception consent form the Ss were asked to begin; and, told that after all s s have participated an explanation of the study would be posted on the sign-up board. 
In order to control for any potential effects of testing condition, each session represented a randomized assignment of subjects to all prime context groups. The only constraint on this assignment was the requirement to equally distribute $\mathbf{s}$ from the personality categories across the prime context groups. There was a master list of names of subjects who were eligible for participation. This list was constructed to both indicate which personality group the subject belonged in, and determine a running log of personality and prime context group assignments. Upon the completion of testing, s from the two personality groups were assigned in a randomized way and approximately equally among the eight prime context groups. After 10 sessions, particular groups that were short on either personality defined subjects or that were too small in general were evened up in the final sessions. Several additional sessions, open to all Introductory Psychology students, were conducted after it was determined that personality was not a significant variable in the prediction. Regardless of grouping constraints, all possible prime context groups were represented in each session.

Results

\section{Replication}

As tests of the replication hypotheses, two 2 (prime cartoon structure) $\times 2$ (switch structure from third to fourth cartoon) $\mathrm{x}$ 4 (trials) MANOVAS were used to analyze the funniness (Figure 5) and aversiveness scores (Figure 6) for the first four cartoons. These analyses yielded replication of the significant ANOVA interaction in the first experiment. However, in contrast to the pattern in experiment 1, NON did not show an extreme end effect enhancement on the fourth trial in these current data. A significant between subjects main effect for prime structure funniness scores, $\underline{E}(1,336)=28.77, \underline{p}<.01$, indicates that NON and INC-RES, as predicted, are different salient structures that are rated for funniness significantly differently. INC-RES was 


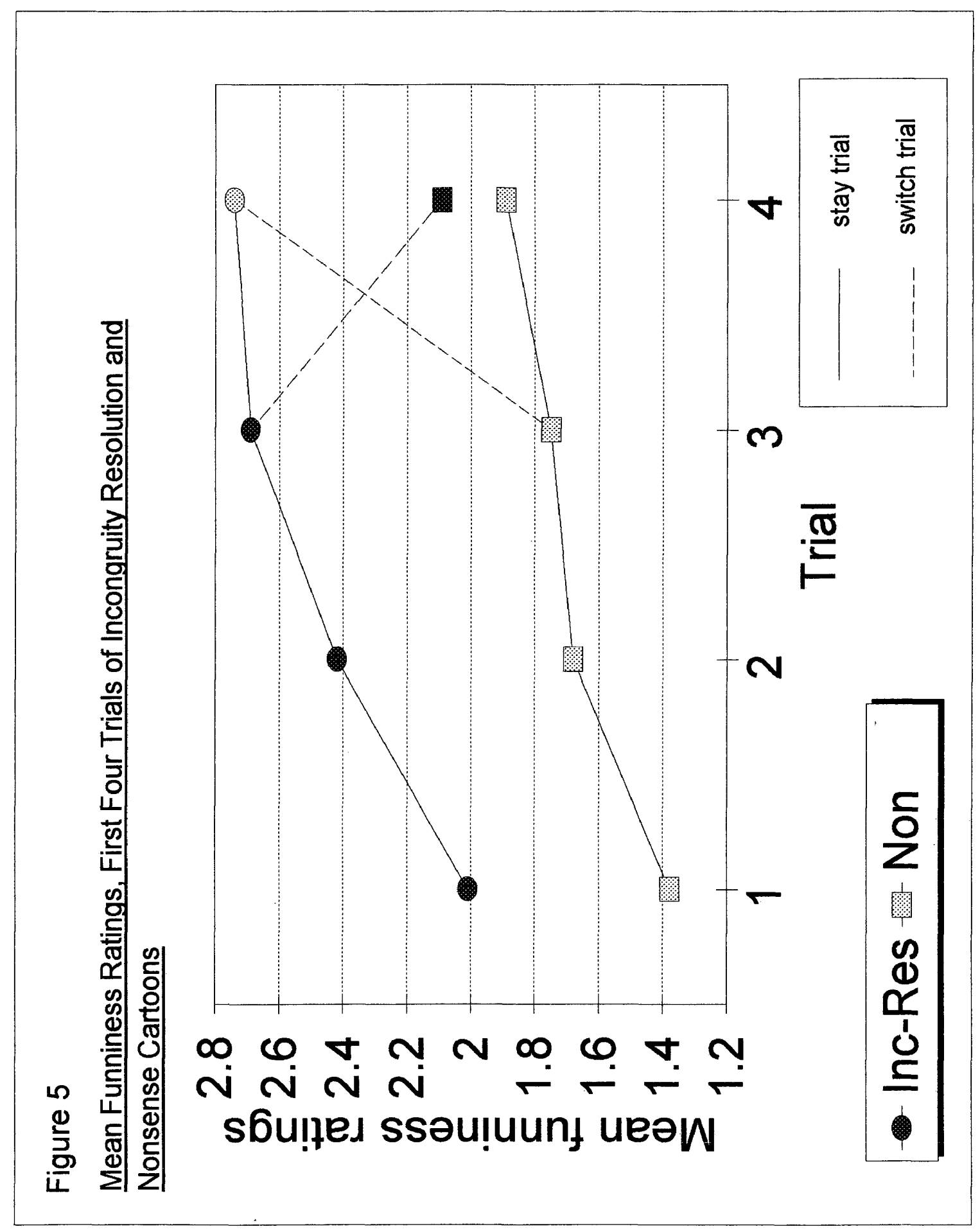


rated as significantly funnier than NON. A significant within subjects main effect for funniness score, $\underline{F}(3,334)=12.07, \underline{p}<.001$ (Pillais approximate F), demonstrates that the scores significantly differ across trials. A significant within subjects interaction between prime structure, switch manipulation and funniness score, $\underline{F}(3,334)=5.10, \underline{p}<$ .01 (Pillais approximate F), signifies that as predicted, funniness scores differ across trials depending upon both prime structure and switch manipulation.

A significant planned comparison ANOVA for funniness scores on the fourth trial results in a significant interaction between prime structure and switch manipulation, $\underline{\mathbf{F}}(1,339)=13.10, \underline{\mathrm{p}}<.001$, which replicates the significant prime by structure interaction in the first experiment. This interaction shows that funniness scores depend upon both which structure is primed and whether a switch to the other structure is introduced on the fourth trial (prime NON switch to INCRES, $m=2.64, n=83 ;$ prime NON stay with NON, $m=1.90, n=86 ;$ prime with INC-RES switch to NON, $m=2.09, \mathrm{n}=86$, and prime with INC-RES stay with INC-RES, $m=2.74, \mathrm{n}=85$ ). However, collapsing across prime groups, a planned comparison t-test for groups rating NON $(n=172)$ and groups rating INC-RES $(\mathrm{n}=168)$ at the fourth trial, $\underline{t}(2,338)=-3.63$, $\underline{p}$ $<.001$, demonstrates that NON $(\mathrm{m}=1.99 ; \mathrm{s.d} .=1.85)$ and INC-RES $(\mathrm{m}=$ $2.69 ; s . d=1.68$ ) are each salient structures that are rated significantly differently for funniness, and regardless of which structure is primed, structure on the fourth trial is rated according to a predictable priming effect. In particular, INC-RES is rated as funnier than NON regardless of switch treatment, but in either case, appreciation is significantly enhanced from the first trial. In Figure 5, NON scores are equivalent on the fourth trial, and INC-RES ratings are comparable on the fourth trial.

Planned comparison ANOVAS for between prime group analyses on the first three trials yield a consistent main effect for prime structure 
indicating that scores on these trials differ by prime structure group: trial 1, $\underline{F}(1,339)=16.05, \underline{p}<.001 ; \operatorname{trial} 2, \underline{F}(1,339)=19.43, \underline{p}<$ $.001 ;$ and, trial $3, \underline{E}(1,339)=16.773, \underline{p}<.001$. INC-RES is consistently rated as funnier than NON. Planned comparison paired ttests, defined by prime structure and assessed between prime trials, were used to evaluate the within subject priming prediction for structure. For both NON, $\underline{t}(1,168)=-2.35, \underline{p}<.01$, (trial 1, $m=1.38$, s.d. $=1.46 ;$ trial $3, m=1.75$, s.d.= 1.67), and INC-RES, $\underline{t}(1,169)=-$ $3.27, \mathrm{p}<.01$, (trial $1, \mathrm{~m}=1.81, \mathrm{~s} . \mathrm{d} .=1.46 ;$ trial $3, \mathrm{~m}=2.34, \mathrm{~s} . \mathrm{d}$. $=1.67$ ), significant priming effects were evident over the first three trials. These results demonstrate that, as predicted, not only NON and INC-RES are unique structures, but also appreciation for humor structure is enhanced by repeated exposure to the single structure prior to a switch manipulation.

The aversiveness analyses (Figure 6) for the first four cartoons produce a similar but less extreme pattern of significant MANOVA results as the funniness scores. A significant between subjects main effect for prime structure, $\underline{F}(1,336)=14.50, \mathrm{p}<.001$, indicates that each structure is uniquely salient and this distinction results in significantly different aversiveness ratings for each structure. INCRES is significantly more aversive than NON. A significant within groups interaction between prime structure, switch manipulation and aversiveness score, $\underline{F}(3,334)=3.99, \underline{p}<.01$ (Pillais approximate $F$ ), indicates that, as predicted, aversiveness scores differ across trials depending upon both prime structure and switch manipulation. A nonsignificant within subjects aversiveness trial effect, $\underline{F}(3,334)=$ $0.385, \underline{p}=.76$ (Pillais approximate $F$ ) shows that aversiveness scores did not differ across priming and switch trials.

Planned comparison ANOVAS indicate a consistent and significant main effect for structure on the first three trials: trial $1, F(1,338)$ $=5.00, \underline{p}<.05$ (NON: $m=.68, n=169 ;$ INC-RES: $m=1.01, \mathrm{n}=171)$; 


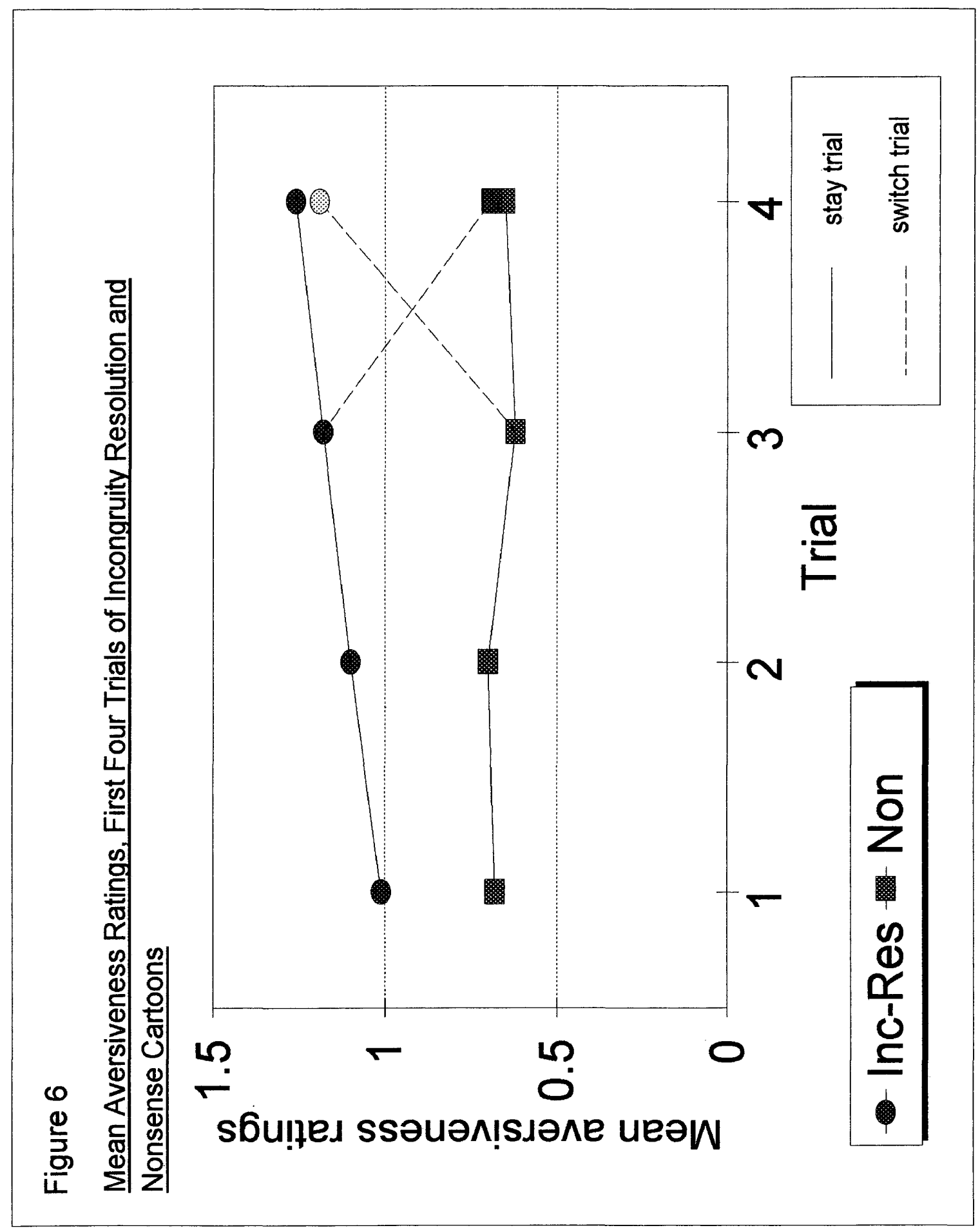


trial 2, $\underline{\mathbf{F}}(1,338)=7.23, \underline{\mathrm{p}}<.001$, (NON: $\mathrm{m}=.70, \mathrm{n}=169$; INC-RES: $\mathrm{m}$ $=1.10, \mathrm{n}=171$ ); and, $\operatorname{trial} 3, \underline{\mathbf{F}}(1,338)=14.53, \underline{\mathrm{p}}<.001$ (NON: $\mathrm{m}=$ $.62, \mathrm{n}=169$; INC-RES: $\mathrm{m}=1.18$ ). On the fourth trial, a significant prime structure by switch manipulation interaction for aversiveness scores, $\underline{F}(1,338)=11.46, \underline{p}<.01$, suggests that aversiveness responses on the fourth trial depend upon which structure is primed and whether a switch is introduced (prime with NON and switch to INC-RES, $m=1.19, \mathrm{n}$ $=83$; prime with INC-RES and switch to NON, $m=.69, \mathrm{n}=86$; stay with NON, $\mathrm{m}=.65, \mathrm{n}=86$; and stay with INC-RES, $\mathrm{m}=1.26, \mathrm{n}=85$ ). However, collapsing across treatment at the fourth trial, a planned comparison $t$-test, $\underline{t}(2,338)=-3.40, \underline{p}<.01$, demonstrates that aversiveness scores for NON $(m=0.67$, s.d. $=1.29, \mathrm{n}=172)$ and INC-RES $(m=1.23$, s.d. $=1.71, n=168)$ are significantly different on the fourth trial. These analyses and nonsignificant paired t-tests between trials indicate that aversiveness scores differ according to structure, but these scores are not altered by repeated exposure.

\section{Full Design}

As analyses of the full design, the seven funniness and aversiveness scores were analyzed by individual 2 (prime cartoon structure) $\times 2$ (personality) $\times 2$ (switch structure from third to fourth cartoon) X 2 (switch structure from third to fifth cartoon) X 7 (trials) MANOVAS. Personality was not a significant factor in the MANOVA analyses; therefore, the "complementary" and the "personality" hypotheses were ruled out. Humor scores in the current experiment were neither a function of an interaction between humor structure, personality and switch manipulation, nor a function of an interaction between humor structure and personality independent of switch manipulation.

Results of the 2 (prime cartoon structure) $\times 2$ (switch structure from third to fourth cartoon) $x 2$ (switch structure from third to fifth cartoon) $\mathrm{x} 7$ (trials) MANOVAS and planned comparison ANOVAS and t-tests 
testing the "state" cognitive hypotheses are discussed for both funniness and aversiveness ratings. Funniness scores (Figure 7) and aversiveness scores (Figure 8 ) in the full design differ depending upon both the specific context created by prime structure (hypothesis 1) and the serial position of the switch manipulation (hypothesis 2 ). Individual group means for funniness and aversiveness ratings are presented in Tables 7 and 8 , respectively. Due to several nonsignificant treatment effects between some groups, Figures 7 and 8 reflect the ratings of all eight groups for each trial, but only the significant differences between treatment groups are presented (i. e., nonsignificant differences are presented as a single point).

Funniness scores are considered first. A significant between groups main effect for prime structure, $\underline{F}(1,332)=6.34, \underline{p}<.01$, indicates that NON and INC-RES are distinctive structures and INC-RES is rated as significantly funnier than NON. A significant between subjects interaction for switch structure from third to fourth cartoon by switch structure from third to fifth cartoon, $\underline{F}(1,332)=4.08, \underline{p}<.05$, demonstrates that funniness scores differ depending upon both whether a switch is introduced in the series and at which trial it is presented in the series. A significant between subjects interaction between prime structure and switch structure from third to fifth cartoon $\underline{F}(1,332)=$ $21.40, \underline{p}<.001$ suggests that funniness scores significantly differ depending upon both which structure is primed and whether there is a switch on the fourth trial and then an immediate switch back on the fifth trial manipulation presented between the third and fifth cartoons in the series.

Significant within subject interactions were also obtained in the overall MANOVA for funniness scores. A significant main effect for score, $\underline{F}(6,327)=9.09, \underline{p}<.001$ (Pillais approximate $F$ ), shows that scores significantly differed over the series. A significant within subjects interaction between prime and score, $\underline{F}(6,327)=8.23, \underline{p}<$ 
Table 7

Mean Funniness Ratings of the Seven Cartoon Trials for the Eight Prime and Switch Manipulation Groups.

PRIME/SWITCH GROUPS

TRIALS

$\begin{array}{lllllll}1 & 2 & 3 & 4 & 5 & 6 & 7\end{array}$

STAY

NON/NON/NON

INC-RES/ INC-RES / INC-RES

$\begin{array}{lllllll}1.4 & 1.7 & 1.8 & 1.9 & 1.8 & 1.9 & 1.8 \\ 2.0 & 2.4 & 2.5 & 2.7 & 2.8 & 2.2 & 2.8\end{array}$

SWITCH/SWITCH BACK

NON / INC-RES / NON

INC-RES/NON/INC-RES

$\begin{array}{lllllll}1.4 & 1.7 & 1.8 & 2.6 * & 1.4 * & 1.9 & 1.5 \\ 2.0 & 2.4 & 2.5 & 2.1 * & 2.6 * & 2.4 & 2.8\end{array}$

SWITCH/STAY

NON/ INC-RES / INC-RES

$\begin{array}{lllllll}1.4 & 1.7 & 1.8 & 2.6 * & 2.6 & 2.7 & 2.7\end{array}$

INC-RES/NON/NON

$\begin{array}{lllllll}2.0 & 2.4 & 2.5 & 2.1 * & 1.4 & 1.7 & 1.7\end{array}$

STAY/SWITCH

$\begin{array}{lllllllll}\text { NON/NON/INC-RES } & 1.4 & 1.7 & 1.8 & 1.9 & 2.6 * & 2.3 & 2.5 \\ \text { INC-RES/INC-RES / NON } & 2.0 & 2.4 & 2.5 & 2.7 & .74 * & 1.3 & 1.3\end{array}$

Note: * indicates a switch structure trial.

Groupings of cartoon presentations mean: "3 trials presented of one structure/ 1 trial presented of one structure/ 3 trials presented of one structure". 


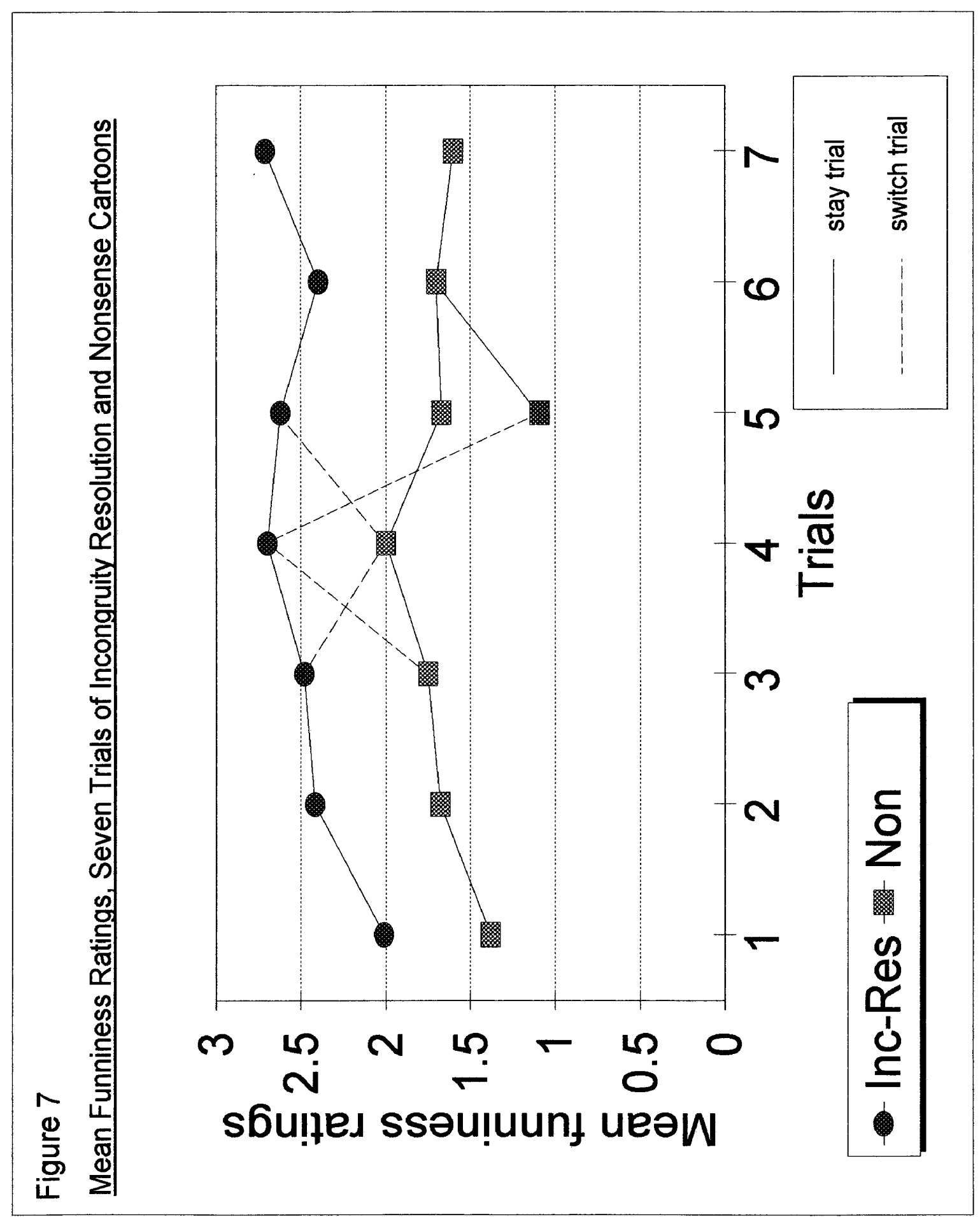


.001 , demonstrates that over the series funniness scores differ by prime structure. A significant within subjects interaction between prime, switch structure from third to fourth cartoon, and score, $\underline{E}(6,327)=$ $3.10, \mathrm{p}<.01$, shows that scores differ across the series depending upon which structure is primed and whether a switch is introduced at the fourth trial. A significant within subjects interaction between prime structure, switch structure from third to fifth cartoon and score, $\underline{F}(6,327)=13.55, \underline{p}<.001$, demonstrates that scores differ across the series as a function of which structure was primed and whether a switch was introduced at the fourth trial and then an immediate switch back was presented at the fifth trial.

Planned comparison ANOVAS and t-tests were employed to test the priming and switch hypotheses for the individual trials. These analyses of funniness ratings indicate both at which trials and what groups are affected by the manipulations. Previously discussed context effects between the first and the fourth trials also apply as pre-requisite effects to these analyses, however they will not be reiterated here. Significant differences. between the NON and INC-RES prime groups (Figure 7) were evident at each trial. Due to the fact that the original error term was used in planned comparison analyses of collapsed treatment groups, significant differences obtained did not require adjustment because the large error variance rendered the conventional significance levels conservative. The specific differences by trial depended upon either prime structure or an interaction between prime structure and switch treatment.

Planned comparison ANOVAS for scores on the fifth through seventh trials consistently show a significant interaction between prime structure and switch manipulations between the third and the fifth trial: fifth trial, $\underline{F}(1,332)=44.14, \underline{p}<.001 ; \operatorname{sixth} \operatorname{trial}, \underline{F}(1,332)=$ 13.58, $\underline{\mathrm{p}}<.001$; and seventh trial, $\underline{\mathrm{F}}(1,332)=32.51, \underline{\mathrm{p}}<.001$. These interactions demonstrate that funniness ratings on the fifth through 
seventh trial depend upon both which structure was primed and whether a switch was introduced on the fourth trial and/or the fifth trial. For current purposes, the fifth trial is the most important result because it is the point in the series where multiple switches should be most salient to the subjects.

Planned comparison t-tests were used to assess the priming and switch hypotheses for the ANOVA treatment groups on the fifth trial. These analyses indicate that funniness scores differ significantly depending upon previous treatment. Subjects primed with NON and then switched to INC-RES on the fifth trial, after either rating NON for four trials or rating INC-RES for three trials and switching to NON on the fourth trial, rated INC-RES $(m=2.58$, s.d. $=1.815, \mathrm{n}=86)$ similarly to those subjects who either were primed with NON for three trials and switched to INC-RES on the fourth trial and stayed with INC-RES for the rest of the series or were primed with INC-RES and stayed with INC-RES $(m=2.66$, s.d. $=1.96, n=86)$ throughout the series, $\underline{t}(2,170)=-.33$, $\underline{p}=.74$. These INC-RES groups were collapsed across treatment groups due to these nonsignificant treatment effects. Appreciation for INC-RES appears to be fairly consistent regardless of exposure to NON on either a single trial or multiple switch trials. NON appears to prime INC-RES without interfering effects. On the other hand, subjects primed with INC-RES and either switched to NON $(m=1.09, \mathrm{~s} . \mathrm{d} .=1.532, \mathrm{n}=85)$ on the fifth trial, after rating INC-RES for four trials or after rating NON for three trials and INC-RES for the fourth trial and then immediately switching back to NON, found it significantly less funny than those subjects primed with NON who stayed with NON (m $=1.63$, s.d. $=1.676, \mathrm{~m}=83$ ) through the series or were primed with INC-RES for three trial and then switched to NoN on the fourth trial, $\underline{t}(2,168)=-$ $2.18, \underline{p}<.05$. Rating INC-RES on the fourth trial interferes with appreciation for NON on the next trial. 
Potential habituation effects at the end of the series were assessed with paired t-tests with groups defined by what individual structure was being rated at that point in the series. Nonsignificant differences, $\underline{t}(1,171)=-.46, \underline{p}=.65$, for funniness ratings on INC-RES between the fifth $(m=2.62$, s.d. $=1.89, n=172)$ and seventh $(\mathrm{m}=$ $2.71, \mathbf{s . d .}=1.96, \mathrm{~m}=172$ ) trials indicate that INC-RES funniness ratings are not significantly altered by the switch manipulations. The suggestion of an end effect can be seen in the marginally nonsignificant difference, $\underline{t}(1,171)=-1.70, \underline{p}=.090$, between INC-RES funniness ratings on trial six $(m=2.39, \mathbf{s} . \mathrm{d} .=1.78, \mathrm{n}=172)$ and trial seven (m $=2.71, \mathrm{~s} . \mathrm{d} .=2.00, \mathrm{n}=172)$. A significant increase in NoN funniness ratings, $t(1,84)=-2.91, p<.01$, between trials five $(\mathrm{m}=1.09, \mathrm{s.d}$. = 1.53, $\mathrm{n}=85)$ and $\operatorname{six}(\mathrm{m}=1.68, \mathrm{s.d} .=1.78, \mathrm{~m}=85)$ suggest the groups that were either primed with NON, exposed to INC-RES for a single trial and then switched back to NON or primed with INC-RES and switched to NON at a later trial recovered to a primed NoN score at the sixth trial. The overall MANOVA for aversiveness scores (Figure 8) indicates some similarity to the funniness ratings results, but the trends are less extremely affected by prime structure and switch manipulations. The pattern of results contradicts the predictions that INC-RES is less aversive than NON because resolution is less incongruous than NON. In addition, the prediction that aversiveness should increase on a switch and decrease as a result of increased structural salience depends upon structure in these data. A significant between subjects main effect for structure, $\underline{F}(1,332)=6.23, \underline{p}<.05$, indicates that INC-RES is generally found more aversive than NON. A significant within group main effect for aversiveness trials, $\underline{F}(6,327)=6.76, \underline{p}<.001$ (Pillais approximate F) demonstrates that scores differ across the series. A significant within subject interaction between prime structure and aversiveness scores, $\underline{F}(6,327)=3.59, \underline{p}<.01$ (Pillais approximate $F$ ), shows that aversiveness scores differ by prime structure. A significant within 
Table 8

Mean Aversiveness Ratings of the Seven Cartoon Trials for the Eight Prime and Switch Manipulation Groups.

$\underline{\text { PRIME/SWITCH GROUPS }}$

TRIALS

$\begin{array}{lllllll}1 & 2 & 3 & 4 & 5 & 6 & 7\end{array}$

STAY

NON/NON/NON

INC-RES/INC-RES/ INC-RES

$\begin{array}{lllllll}.68 & .70 & .62 & .65 & .26 & .26 & .29 \\ 1.0 & 1.1 & 1.2 & 1.3 & .49 & 1.1 & .42\end{array}$

SWITCH/SWITCH BACK

NON / INC-RES / NON

$\begin{array}{llllllll}.68 & .70 & .62 & 1.2 * & .24 * & .51 & .20\end{array}$

INC-RES/NON / INC-RES

$\begin{array}{llllllllll}1.0 & 1.1 & 1.2 & .69 * & .95 * & .74 & .70\end{array}$

SWITCH/STAY

NON / INC-RES / INC-RES

$\begin{array}{lllllll}.68 & .70 & .62 & 1.2 * & .90 & 1.1 & 1.0\end{array}$

INC-RES/NON/NON

$\begin{array}{llllllll}1.0 & 1.1 & 1.2 & .69 * & .47 & .70 & .58\end{array}$

STAY/SWITCH

NON / NON / INC-RES

$.68 \quad .70 \quad .62 \quad .65 \quad .95 * \quad 1.0 \quad .77$

INC-RES/INC-RES/NON

$\begin{array}{lllllll}1.0 & 1.1 & 1.2 & 1.3 & .29 * & .50 \quad .33\end{array}$

Note: * indicates a switch structure trial.

Groupings of cartoon presentations mean: "3 trials presented of one structure/ 1 trial presented of one structure/ 3 trials presented of one structure". 


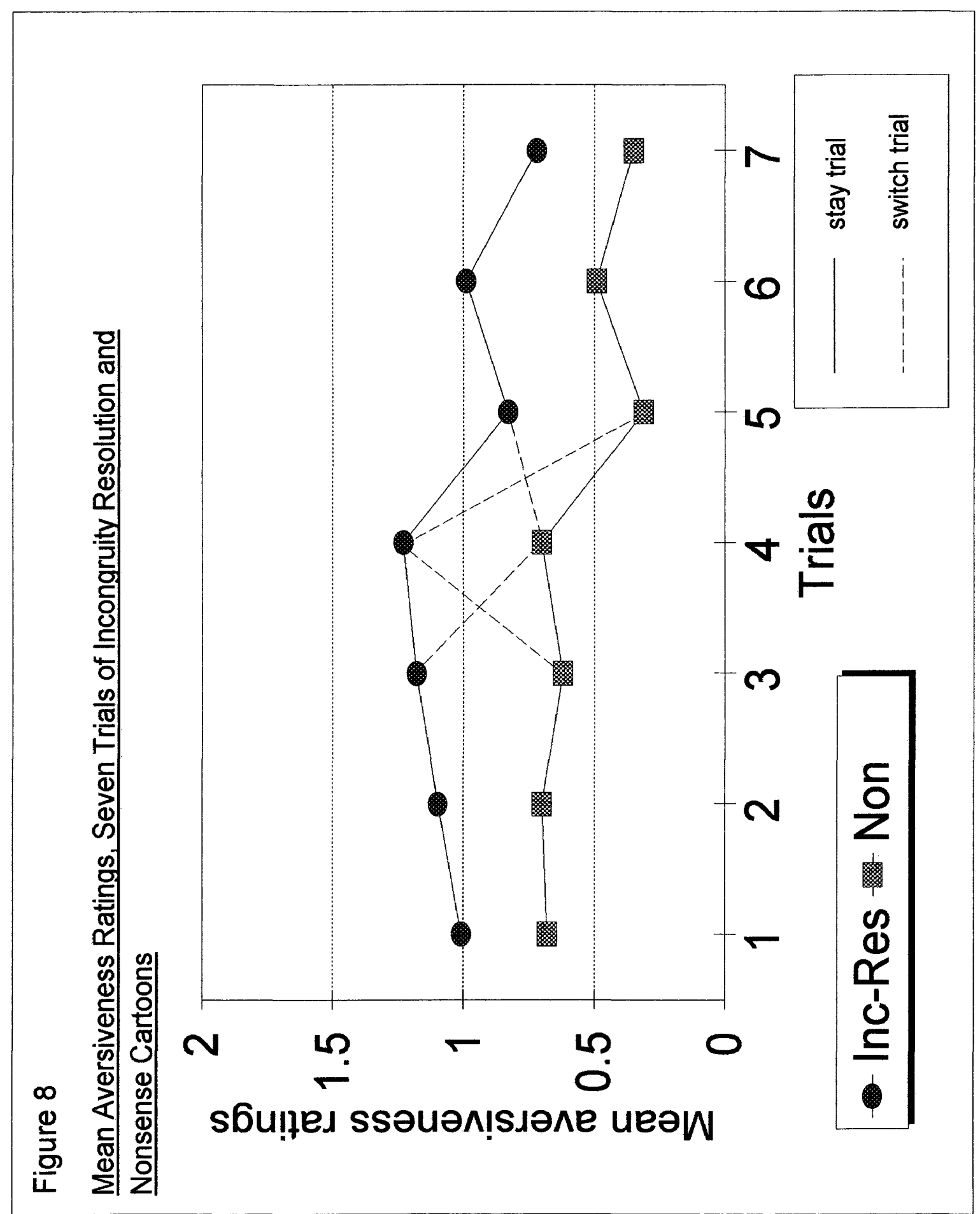


subjects interaction between prime structure, switch manipulation between the third and fourth trials, and aversiveness score, $\underline{F}(6,327)=$ $3.07, \underline{p}<.001$ (Pillais approximate F), indicates that aversiveness scores differ depending upon the prime structure and whether a switch is introduced in the series at the fourth trial. A significant within subjects interaction between switch manipulation between the third and fourth trials, switch manipulation between the third and fifth trials and aversiveness scores, $\underline{F}(6,327)=2.43, \underline{p}<.05$ (Pillais approximate F), demonstrates that primarily based on prime structure, aversiveness scores depend upon whether the switch is introduced at the fourth trial, the fifth trial, or whether the switch and switch back manipulation is presented.

Planned comparison ANOVAS for the fifth through seventh trials reveal significant interactions between prime structure and switch treatment between the third and fifth trial: trial 5, $\underline{\mathbf{F}}(1,332)=15.25$, $\underline{\mathrm{p}}<.001 ; \operatorname{trial} 6, \underline{\mathrm{F}}(1,332)=10.88, \underline{\mathrm{p}}<.001 ;$ and $\operatorname{trial} 7, \underline{\mathrm{F}}(1,332)=$ $8.77, p<.01$ which indicate that aversiveness scores differ depending upon the pattern of switch treatment between the third and fifth trials. However, the switch cartoons are rated consistently according to prime structure differences in aversiveness. Switching from NON to INC-RES increases aversiveness ratings, but switching from INC-RES to NON decreases aversiveness ratings. These mixed results do not support the predictions that switching structure should increase aversiveness ratings, and priming structure should decrease aversiveness ratings. In these data, aversiveness ratings are predictable according to a main effect for structure. The meaningfulness of these results is suspect because INC-RES is always rated more aversive than NON.

Discussion

It is clear that subjects detect a difference between the structures because there is both a consistent main effect for prime structure, and specific manipulations serve as context variables that do 
significantly alter scores. Differences in predictability, the stimulative value of the structure itself, is a relevant factor in appreciation scores. Ruch (1992) indicates that the unresolved incongruity of NON is more arousing than the resolved incongruity of INC-RES. In these current data, the perception of novelty in NON may be arousing, but it seems to have an inhibitory effect on appreciation that is reflected in the consistent and significantly lower funniness ratings for NON relative to INC-RES. The difference in appreciation ratings given to INC-RES and NON supports a "salience" hypothesis where the salient dimension of the stimuli is the resolution of incongruity given by the structure.

Planned paired t-test comparisons for individual trials indicate that priming enhanced appreciation for both INC-RES $(\mathrm{n}=180)$ and NON ( $\mathrm{n}$ = 179) when each structure was presented alone. These results support hypotheses 1 , the prediction that appreciation for the single structure may be enhanced through repeated exposure to that single structure. These priming results support Forabosco's (1991) "context hypothesis" sequence rule that appreciation for structure is higher when the presentation of humor stimuli establishes a structural link between cartoons.

In the full design, the control groups (NON, $n=42$; INC-RES $=43$ ) beyond the third trial, perhaps had insufficient power to support either a "salience" hypothesis (Goldstein, 1973) or the "dual process" (Deckers et al., 1989) hypothesized response sensitization each predicting increasing funniness scores over the full series. In the full design, the single cartoon groups are two of eight groups. Any continuous sensitization and habituation effects that may have occurred across the series were probably too subtle for detection by the relatively small control groups. The aversiveness main effect for structure indicates that INC-RES is more aversive than NON and the nonsignificant within subjects effect for aversiveness trial suggests that aversiveness scores 
differ according to prime structure but not a context effect of differing structural presentation across the series.

These current data may only tentatively address the "dual process" hypothesis (Deckers et al., 1989) prediction for funniness scores progressively increasing due to sensitization, and then decreasing beginning at some score in the series because of response habituation. Response sensitization or enhanced salience is defined as a process where a "cognitive set" for a stimulus characteristic facilitates processing of like stimuli and positively affects humor appreciation (Goldstein et al., 1972). Response habituation is conceptualized as a decrease in appreciation that occurs between the third and the fifth cartoon (Deckers, et al., 1989). The results of an experiment in progress (Derks \& Staley, 1993) may better determine the role of sensitization and habituation effects in structure cartoon series. The significant interactions between and within subjects for the various switch/prime manipulations indicate that the switch structure manipulations are responsible for the facilitation and interference of processing in these data.

The fact that the switch manipulations were introduced during the predicted habituation period (Deckers et al., 1989) render these results most theoretically relevant to the conditions of structural salience. The significantly different rating of the "switch" cartoon from the priming cartoons supports a "salience hypothesis" for humor structure. These results indicate that the specific juxtaposition of structure is both a relevant context variable in a cartoon series and an influential factor in preference. Priming structure enhances the salience of that structure and increases appreciation for the "pre-switch" cartoons. The primed structure creates a context in which the "switch" cartoon is perceived (hypothesis 2A). This context both affects ratings of the "switch" cartoon and subsequent "switch" or "stay" cartoons (hypothesis 2B). Enhanced or diminished ratings for the "switch" and subsequent 
cartoons depends upon the interaction between prime structure and treatment between the third and fifth trials.

Hypothesis $2 \mathrm{~A}$ is a "salience hypothesis" which predicts that the "switch" cartoon on the fourth trial should be rated significantly differently from the different structure cartoons with which it was primed. This first "switch" condition significantly replicates the general pattern of Experiment 1. These results suggest that each structure is unaffected by negative prime. Results from Experiment 1 show scores dropping when INC-RES ("prime") primes NON ("switch"), rising when NON ("prime") precedes INC-RES ("switch"), and increasing from NON to NON on the final trial (Figure 3). However, in Experiment 1, repeated exposure to NON ended in a sharply enhanced appreciation for NON on the final trial. In Experiment 2, the NON only group showed consistent primed scores but not an end effect interaction. Experiments 1 and 2 clearly differ in the cartoon series length, and this difference may explain the conflicting results. An end effect is also suggested in experiment 2 for INC-RES. However, an end effect for both NON and INCRES depending upon the length of the series is not a strong explanation. The consistency of these end effects should be empirically demonstrated.

A planned comparison ANOVA for these current data indicates a significant interaction between prime and switch structure at fourth cartoon showing that funniness scores for NON return to a primed NON score after exposure to INC-RES and scores for INC-RES rise to a primed INC-RES score after priming with NON. The prime cartoons structure is salient prior to the "switch" because the "switch" cartoon is rated significantly differently from the prime cartoons. In Goldstein's et al. (1972) terms, it appears that a cognitive set for INC-RES may subtly interfere with processing the NON "switch" cartoon. Priming with INCRES may thus interfere with processing NON, but priming with NON appears to facilitate the processing of INC-RES. However, the explanation of interfering and facilitating effects of contrasting structural effects 
must be further tested in a latency analysis. The conclusion that may be drawn from these data is that on the first four trials, appreciation scores differ by prime structure. The scores for NoN are not significantly different due to switch treatment at the fourth trial, nor are the scores for INC-RES significantly different because of treatment at the fourth trial. This lack of a specific effect suggests that a general priming effect of exposure to humor enhances scores according to a "general incongruity factor" for each structure.

As shown in Experiment 1 and significantly replicated in Experiment 2, if structures are presented in contrast, then priming with the more congruous structure (INC-RES) seems to interfere with the funniness of the less congruous structure (NON), but priming with the less congruous (NON) does not seem interfere with appreciation for the more congruous (INC-RES). The significance and consistency of this pattern strongly contradicts Forabosco's (1991) sequence rule that presentation from the more congruous to the less congruous is the preferred construction for highest appreciation scores. However, the hypothesized interference and facilitation of processing predicted as responsible for the contrast effect require empirical demonstration. Hypothesis 2A, of the "salience hypothesis", also predicts that the "switch" cartoon on the fifth trial should be rated significantly differently from both the first "switch" cartoon and the structure cartoons with which it was primed. Hypothesis $2 \mathrm{~B}$ addresses the effect of the second "switch" cartoon ("switch/back") on appreciation for subsequent humor stimuli. The effects of the "switch/back" cartoon in a series of "prime structure/ switch structure/switch back to primed structure" were the primary interest of the second experiment because the "switch/back" cartoon added a new manipulation to the design of Experiment 1. With groups collapsed across the switch at the fourth cartoon category, planned comparison ANOVA results for the fifth trial indicate that the significant pattern of responses to the "switch" 
structure at the fourth trial and the immediate "switch/back" to the primed structure at the fifth cartoon is laterally inverted by structure (Figure 7).

There is a significant treatment difference between subjects rating NON and subjects rating INC-RES at the fifth trial. Subjects rating INC-RES on the fifth trial did not differ in regard to previous treatment. These results demonstrate that appreciation for INC-RES may be primed by NON without interference, and across the series, the juxtaposition of INC-RES and NON does not decrease appreciation for INCRES. Subjects primed with NON who rated INC-RES on the fifth trial (m = 2.58, $\mathrm{n}=86$ ) and subjects primed with INC-RES who rated INC-RES on the fifth trial ( $m=2.66, n=86$ ) did not significantly differ. However, the most interesting result in the present experiment is that the subjects ratings of NON on the fifth trial depended upon the context created by previous treatment. Subjects who rated NON on both the fourth and fifth trials. ( $m=1.63, \mathrm{n}=83$ ) found it significantly funnier than subjects who rated INC-RES on the fourth trial and then rated NON on the fifth trial ( $m=1.09, \mathrm{n}=85)$.

The effects of INC-RES presentation on appreciation for NON may be described by a "contrast" hypothesis. Exposure to INC-RES for four priming trials shows enhanced appreciation for INC-RES which suggests that a "cognitive set" has been established for an expectation of resolution of incongruity. According to Goldstein et al. (1972), the cognitive set enhances appreciation by facilitating the processing of like stimuli. In terms of dual process theory ss may rapidly habituate to INC-RES and form a cognitive set for INC-RES, and the switch to NON is an interference to processing. Priming can inhibit processing of subsequent stimuli. This interference may confound understanding and thus, results in decreased appreciation. Comprehension is a prerequisite for appreciation (Bariaud, 1989). Introducing NON in the context of INC-RES violates the expectation of resolution and inhibits 
appreciation or acts as a "contrast" which re-sets the subject below unprimed scores on NON. If salient stimuli may be either be common or unusual, then priming with the common structure may render the unusual less accessible. Alternatively, because the single presentation of INCRES, in the context of NON, also yields a significantly lower than preprimed score on the subsequent NON presentation, INC-RES may be uniquely salient. The "contrast" effect of INC-RES in the context of NON may alter subjects' perspective on funniness. In this view, the standard of funniness is re-set to include the now salient and readily appreciated quality of resolution. As collaborating evidence for these results being due a structural context effect, and not the result of INC-RES merely being funnier than NON, Derks and Arora (1993) report that low quality humor is found funnier when it is primed by high quality humor. In terms of these current data, the significantly lower appreciation for NON in the context of INC-RES may be attributed to the salience of a different structure being introduced into the series. If these results were due to a low humor/high humor effect, then NON should have been rated significantly more funny not significantly less funny following priming with INC-RES.

\section{General Discussion}

Incongruity has a long philosophical heritage as a primary feature of humor. Psychological incongruity theories of humor build upon these philosophical definitions and demonstrate that incongruity and resolution of incongruity are the structural elements of humor which give form to the content of the humor stimulus (McGhee, 1979; Nerhardt, 1977; Rothbart \& Pien, 1977; Ruch, 1992; Shultz, 1972; and, Suls, 1972). Comprehension, simultaneity, tolerance and context are the complementary processing variables that render the perception of incongruity humorous (Bariaud, 1989; Deckers et al., 1989; Derks \& Arora, 1990; Forabosco, 1991; Goldstein et al., 1972; Leventhal \& Safer, 1977; and Ruch, 1992). In a final analysis, a comprehensive explanatory theory of humor 
appreciation will predict the specific effects of the processing variables; explain how the two types of structural incongruity are differentially perceived and processed as humorous; predict how humor content affects humor appreciation; and define what individual and contextual conditions will render the various combinations of content and structure relatively both more and less amusing. The aim of the current studies was to attempt to make a small contribution to that goal by evaluating the effects of the processing variables tolerance for ambiguity and structural context on humor appreciation.

The conceptual basis for the currently reported studies was that incongruity, as defined by a Gestalt view, has multi-dimensional theoretical effects on humor appreciation. "In a Gestalt view, the meaning of an element or group of elements depends upon the whole configuration of which it is a part. Changing only a single part of the whole may change the meaning not only of the part, but of the whole as well" (McGhee, 1979, p. 11). The Gestalt relationships between meaning and configuration may apply to both the particular structures of NON and INC-RES as individual presentations of incongruity, and the juxtaposition of these structural configurations of incongruity in multiple presentation. The individual structure and the serial presentation of structures each form a particular context of incongruity. As the differing stimulative value of the presence or the absence of resolution of incongruity is a stimulus context that alters appreciation scores (Ruch, 1992), so does the context created by the particularities of multiple structural presentation influence appreciation ratings (Forabosco, 1991).

The current three studies were conducted specifically to evaluate the distinction that NON and INC-RES are independent humor structures that may be appreciated as humorous differently. The first correlational study failed to replicate Ruch's (1992) personality based predictions concerning tolerance for ambiguity as a determinant of humor 
preference. Although structural salience was not a significant factor in the assessment of Ruch's (1992) personality predictions in the correlational study, the pattern of funniness responses (Figure 1) indicates a tendency for INC-RES to be rated as funnier than NON. The random pattern of aversiveness ratings rendered the validity of this measure of humor appreciation suspect. It was concluded that the conditions under which Ruch's (1992) predicted differences in appreciation for humor structure are evident should be experimentally determined.

Experiments 1 and 2 assessed context effects, structure, and personality based tolerance for ambiguity as either independent or complementary processes in appreciation for humor structure. Results of the first experiment suggest that a personality based tolerance for ambiguity has moderating effects in appreciation for humor structure presented in series. Significant difference scores analyses (Table 3) suggest that Sensation Seekers (Zuckerman, 1979) may be more susceptible, than Conservatives (Wilson, 1973), to habituation in funniness ratings when a single structure cartoon is primed. Significant correlations (Tables 4 and 5 ) suggest that Sensation Seekers (Zuckerman, 1979) may find NON significantly more amusing than Conservatives (Wilson, 1973), and Conservatism (Wilson, 1973) predicts aversion for INC-RES. A relative funniness for structure is also suggested by the correlations (Table 5) that Conservatism (Wilson, 1973) is positively related to funniness for INC-RES, and sensation seeking (Zuckerman, 1979) is negatively related to funniness for INC-RES. As a general conclusion, these results both support Ruch (1992), and suggest that tolerance for ambiguity is related to appreciation for humor structure.

However, the second experiment ruled out personality as a significant influence in appreciation when content is a controlled variable and cartoon presentation is manipulated. Nevertheless, the 
grouping method used to define personality in Experiment 2 may have prevented the variance in tolerance for ambiguity to be adequately assessed as a significant variable in appreciation for structure. In particular, the personality variables may account for some of the main effect difference between structure preference (Figures 7 and 8 ). Future research could assess this possibility perhaps by using personality as a covariate in a context effect analysis.

Ruch's (1992) conclusion that the differing stimulative natures of NON and INC-RES are important determinants of appreciation for humor structure receives support from both the main effect for prime structure in Experiments 1 and 2, and the relationships between tolerance for ambiguity and structural appreciation in Experiment 1. These collaborating conclusions suggest that further assessment of the conditions under which a moderating effect of tolerance for ambiguity is most influential in appreciation for humor structure is justified.

Results of the two experiments help clarify how structural context, manipulated through priming and strategic switching of structures, contributes to humor appreciation. The experimental results clearly and significantly support the pattern of responses suggested in the correlational study which indicate that INC-RES and NON are both uniquely perceived structures, and INC-RES is generally found funnier than NON. In Experiments 1 and 2, INC-RES was also rated as significantly more aversive than NON. Because INC-RES is a common form of humor, it is not unusual that INC-RES was rated as funnier than NON, but it is peculiar that INC-RES was also consistently rated as more aversive. Although funniness and aversiveness are theoretically independent ratings of humor structure, aversiveness should be higher for pure incongruity than for resolved incongruity. These results question the validity of the aversiveness measure which indicates that aversiveness requires clearer specification and further empirical evaluation in order to be a useful measure of humor appreciation. The 
results of Derks and Staley (1993) may help define the empirical relationship between aversiveness and appreciation for humor structure. Forabosco's (1991) context effect "sequence rule" predicting that maximum appreciation for a series of differing humor structures depends upon a presentation that starts with the more congruous INC-RES and introduces the less congruous NON later in the series is strongly contradicted by the results of Experiments 1 and 2 . However, Forabosco's (1991) second rule that predicts maximum appreciation for cartoons in a series when a structural link is established between cartoons is supported by the enhanced appreciation seen in experiment 2 when a single structure was primed. The consistency of the obtained context effects over a series of more than seven cartoon is not known, and future research may determine how context effects of juxtaposing NoN and INC-RES in series continues to or ceases to influence humor appreciation responses.

In regard to the stimulus salience and response habituation predictions, only structural salience is strongly supported by the results of Experiments 1 and 2. Salience between structures is evident by the consistent main effect for structure, and increased salience for the single structure is. seen in the effects of priming on structure scores. Goldstein et al. (1972) predicts that salience enhances appreciation because the establishment of a cognitive set facilitates the processing of like stimuli. It is not clear in these current data whether a cognitive set can explain the results because facilitation and interference effects were not measured directly; consequently, a future latency study may specifically test this prediction. According to "dual process" theory (Deckers et al., 1989), response habituation should decrease appreciation within a series of same structure cartoons between the third and the fifth presentation. However, this effect can only be weakly suggested in these current data, and future assessments of single structure presentations may evaluate not only when an habituated 
appreciation for structure occurs, but also if this adaptation process is different depending upon the particular structure of incongruity. The empirically and theoretically consistent main effect difference between funniness ratings for NON and INC-RES may have been sufficient to explain appreciation of humor structure. However, in the second experiment, funniness for NON was found to depend upon whether or not INC-RES was presented in the series as a contrast structure (Figure 7). Contrary to the potential facilitating effects of salience, increasing salience also inhibited appreciation in these present data when a switch to NON was introduced in a series of INC-RES. This result demonstrates that the context created by particular structural juxtaposition should be considered as a factor in appreciation scores for NON. The conclusion that this is an effect of incongruity and incongruity resolution contrast, not a high/low humor effect, is supported by results reported by Derks and Arora (1993). Derks and Arora (1993) demonstrated that low quality humor is rated funnier when it is primed with high quality humor. Future replications could employ both different INC-RES and NON cartoons and jokes in a context experiment, and test these variables in different humor mediums in order to determine if the obtained results reflect general effects of structural context.

The interaction between structure and switch manipulation and the effect on NON scores is relevant to interpretations of humor study results where both structures are interwoven in a series of presentations. Not distinguishing between the two structures may lead to misinterpretation of results. Relevant to a Gestalt description of the humor stimuli (McGhee, 1979), the "context hypothesis" (Forabosco, 1991) as it applies to humor structure may offer a potential explanation of the failure of attempts to replicate Goldstein, et al (1972). This would be relevant if the original study and the replications tested similar themes but used different types of structural presentations. 
Lowering the threshold for particular content may liberate processing capacity for more sensitive detection of other elements of the stimuli such as structure. If this was the case, then primed responses in both Goldstein et al (1972) and the replication attempts would reflect an interaction between increased salience of a particular theme and perhaps a heightened perception of structural distinctions within a series. A replication of Goldstein et al (1972) including their identical presentation of structural configuration and manipulating content presentation would be useful for assessing the veracity of his conclusions concerning the effects of content salience on humor appreciation. In addition, a computer generated reaction time analysis may help assess not only the usefulness of the facilitating and interfering predictions of structural contrast, but also may determine whether Goldstein's et al. (1972) predictions for the effects of salience and facilitation of processing in terms of cognitive sets are adequate for explaining several of the results of these current priming and switching structure experiments.

Taken as a whole, the results of the current studies suggest that structural distinctions, tolerance for ambiguity, and a context created by the particular structural presentation interact in humor appreciation. In addition, the potential complexity of interactions between content, structure and the precise configuration of both elements of humor in a series of jokes and cartoons indicates that these interactions, between the elements of the humor stimuli themselves, affect the perception of incongruity and therefore humor appreciation. The demonstration of context effects of humor structure and theme presentation are not only relevant considerations in current humor research, but also collaborating support for cognitive incongruity theories of humor appreciation.

In terms of a comprehensive theory of humor appreciation, the current results are a small but useful step towards describing some of 
the specific effects of the processing variables emotional tolerance and cognitive context. However, this small advance indicates that additional research both assessing the hypothesized explanatory power of interfering and facilitating effects of established cognitive sets for structure in appreciation for primed and juxtaposed structural presentation, and evaluating predicted habituation effects in humor appreciation should be undertaken. Further, these present results suggest that additional specification of both the roles of structural incongruity and resolution in humor appreciation, and the potential effects of individual differences variables, such as cognitive tolerance for ambiguity, are justified.

In conclusion, the current results support previous research and theory predictions concerning the relevance of context effects of humor presentation in humor appreciation (Deckers et al., 1989; Derks \& Arora, 1990; Forabosco, 1991; and, Goldstein et al., 1972). Demonstrating that humor appreciation as a multi-dimensional phenomenon has implications for humor theory and ramifications for the appropriate use of humor in clinical and educational settings. In particular, showing that context effects are influential in humor appreciation renders the Freudian drive reduction explanation of humor appreciation inadequate. Additionally, applied uses of humor should be undertaken with knowledge of the empirically demonstrated interacting effects of individual differences, humor content and structure of incongruity on humor appreciation. Such an understanding of humor appreciation will help ensure not only that humor is used most effectively in therapeutic and educational application, but also and perhaps more importantly, that humor is employed so as to avoid having negative consequences in mental health interventions and educational uses. 
References

Bariaud, F. (1989). Age differences in children's humor. In P. E. MCGhee (Ed.), Humor and Children's development (pp. 15-45). New York: The Hawthorn Press.

Berlyne, D. E. (1972). Humor and its kin. In J. H. Goldstein \& P. E. MCGhee (Eds.), The Psychology of Humor (pp. 43-59) . New York: Academic Press.

Cacioppo, J. T., \& Petty, R. E. (1982). The need for cognition. Journal of Personality and Social Psychology, 42(1), 116-131.

Deckers, I., Buttram, R. T., \& Winsted, D. (1989). The sensitization of humor responses to cartoons. Motivation and Emotion, 13(1), 71-81. Derks, P., \& Arora, D. (1993). Sex and salience in the appreciation of humor. Humor, $\underline{6}(1), 57-69$.

Derks, P., \& Staley, R. (1993). A test of a well formed theory of humor elicitation (and a bunch of not so well formed ones). experiment in progress.

Forabosco, G. (1991). "Seriality" and appreciation of jokes. Paper presented at the Ninth International Conference on Humour and Laughter, Brock University, Canada.

Goldstein, J. H., Suls,.J. M., \& Anthony, S. (1972). Enjoyment of specific types of humor content: Motivation or salience ? In Goldstein, J. H. \& P. E. McGhee (Eds.), Psychology of Humor, (pp. 159-171). New York: Academic Press.

Johnston, W. A., \& Dark, V. J. (1986). Selective attention. Annual Review of Psychology, 37, 43-75. Leventhal, H., \& Safer, M. A. (1977). Individual differences, personality, and humour appreciation: Introduction to symposium. In A. J. Chapman \& H. C. Foot (Eds.), It's a Funny Thing, Humour (pp.335-349) . Oxford: Pergamon Press. McGhee, P. E. (1979) Humor it's origin and development. San Francisco: W. H. Freeman and Company. 
Morreall, J. (1989). Enjoying incongruity. Humor, $\underline{2}(1), 1-18$. Nerhardt, G. (1977). Operationalization of incongruity in humour research: A critique and suggestions. In A. J. Chapman \& H. C. Foot (Eds.), It's a Funny Thing, Humour (pp.47-51). Oxford: Pergamon Press.

Rothbart, M. K., \& Pien, D. (1977). Elephants and marshmallows: A theoretical synthesis of incongruity-resolution and arousal theories of humour. In A. J. Chapman \& H. C. Foot (Eds.), It's a Funny Thing, Humour (pp.37-40). Oxford: Pergamon Press.

Ruch, W. (1983). Humor-test 3 WD (Form A and B). Unpublished manuscript. University of Dusseldorf, Department of Psychology, Dusseldorf, FRG.

Ruch, W., \& Hehl, F. J. (1986a). Conservatism as a predictor of responses to humor-I. A comparison of four scales. Personality and Individual Differences, I, 1-14.

Ruch, W. \& Hehl, F. J. (1986b). Conservatism as a predictor of responses to humor-II. The location of sense of humor in a comprehensive attitude space. Personality and Individual Differences, $\underline{7}, 861-874$.

Ruch, W. (1988). Sensation seeking and the enjoyment of structure and content of humour: Stability of findings across four samples. Personality and Individual Differences, 9 , 861-871.

Ruch, W. (1992). Assessment of appreciation of humor: Studies with the 3 WD humor test. C.. D. Spielberger \& Butcher (Eds.), Advances in Personality Assessment, (vol. 3). L.E.A. Hillsdale. Ruch, W., Ott, C., \& Bariaud, F. (1991). Cross-national comparison of humor categories: France and Germany. Humor, $\underline{4}(3 / 4), 391-414$. Shultz, T. R. (1972). The role of incongruity and resolution in children's appreciation of cartoon humor. Journal of Experimental Child Psychology, 13, 456-477. 
Suls, J. M. (1972). A two-stage model for the appreciation of jokes and cartoons: An information-processing analysis. In Goldstein, J. H., \& McGhee, P. E. (1972), The Psychology of Humor (pp. 81-99). New York: Academic Press.

Wyer, R. S. \& Collins, J. E. (1992). A theory of humor elicitation. Psychological Review, 99(4), 663-688.

Wilson, G. D. (Ed.). (1973). The psychology of conservatism. New York: Academic Press.

Zuckerman, M. (1979). Sensation seeking: Beyond the optimal level of arousal. New York: Lawrence Erlbaum Associates, Publishers. 


\section{APPENDICES}

Appendix

Page

1. The Zuckerman (1979) Sensation seeking scale........... 92

2. The Cacioppo and Petty (1982) Need for Cognition Scale..... 95

3. The Wilson (1973) Conservatism Scale, as Modified for

Use in the Current studies.................... 97

4. Jokes and Cartoons from the 3WDA and 3WDB Humor Scale (Ruch,

1983) Used in the Three Studies. Note: All jokes are

INC-RES in the 3WD Humor scale (Ruch, 1983).

a. Jokes and cartoons \#1 through \# 36 were used in the

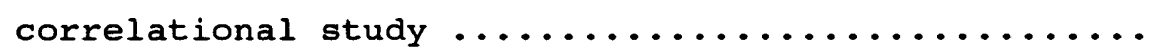

b. In the next series of seven cartoons, all of the cartoons are NON. The first four were used in both experiments, and the last three were only used in

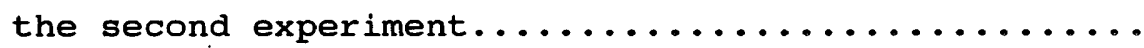

c. In the last series of seven cartoons, all of the cartoons are INC-RES. The first four were used in both experiments, and the last three were only used in

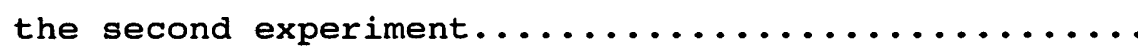


Directions: Each of the items below contains two choices: $A$ and $B$. Please indicate which of the choices best describes your likes or dislikes or the way you feel. In some cases you may find items in which both choices describe your likes or the way you feel. Please choose the one that better describes your likes or feelings. In some cases you may find items in which you do not like either choice. In these cases mark the choice you dislike the least.

It is important that you respond to all items with only one choice: A or B. We are interested only in your likes or feelings, not in how others feel about these things or how one is supposed to feel. There are no right or wrong answers. Try to give an honest appraisal of yourself.

1. A. I like "wild" uninhibited parties.

B. I prefer quiet parties with good conversation.

2. A. There are some movies I enjoy seeing a second time or even a third time.

B. I can't stand watching a movie that I've seen before.

3. A. I often wish I could be a mountain climber.

B. I can't understand people who risk their necks climbing mountains.

4. A. I dislike all body odors.

B. I like some of the earthy body smells.

5. A. I get bored seeing the same old faces.

B. I like the comfortable familiarity of everyday friends.

6. A. I like to explore a strange city or section of town by myself, even if it means getting lost.

B. I prefer a guide when I am in a place I don't know well.

7. A. I dislike people who do or say things just to shock or upset people.

B. When you can predict almost everything a person will do or say he or she must be a bore.

8. A. I usually don't enjoy a movie or play where I can predict what will happen in advance.

B. I don't mind watching a movie or play where I can predict what will happen in advance.

9. A. I have tried marijuana or would like to.

B. I would never smoke marijuana.

10. A. I would not like to try any drug which may produce strange

and dangerous effects on me.

B. I would like to try some of the drugs that produce hallucinations.

11. A. A sensible person avoids activities that are dangerous.

B. I sometimes like to do things that are a little frightening.

12. A. I dislike "swingers".

B. I enjoy the company of real "swingers".

13. A. I find that stimulants make me uncomfortable.

B. I often like to get high (drinking liquor or smoking marijuana). 
14. A. I like to try new foods that I have never tasted before.

B. I order the dishes with which I am familiar, so as to avoid disappointment and unpleasantness.

15. A. I enjoy looking at home movies or travel slides.

B. Looking at someone's home movies or travel slides bores me tremendously.

16. A. I would like to take up the sport of water-skiing.

B. I would not like to take up water-skiing.

17. A. I would like to try surfing.

B. I would not like to try surfing.

18. A. I would like to take off on a trip with no pre-planned or definite routes or timetable.

B. When I go on a trip I like to plan my route and timetable fairly carefully.

19. A. I prefer the "down-to-earth" kinds of people as friends.

B. I would like to make friends in some of the "far-out" groups like artists and hippies.

20. A. I would not like to learn to fly an airplane.

B. I would like to learn to fly an airplane.

21. A. I prefer the surface of the water to the depths.

B. I would like to go scuba diving.

22. A. I would like to meet some persons who are homosexual (men or women).

B. I stay away from anyone I suspect of being "queer".

23. A. I would like to try parachute jumping.

B. I would never want to try jumping out of a plane with or without a parachute.

24. A. I prefer friends who are excitingly unpredictable.

B. I prefer friends who are reliable and predictable.

25. A. I am not interested in experience for its own sake.

B. I like to have new and exciting experiences and sensations even if they are frightening, unconventional, or illegal.

26. A. The essence of good art is in its clarity, symmetry or form and harmony of colors.

B. I often find beauty in the "clashing" colors and irregular forms of modern painting.

27. A. I enjoy spending time in the familiar surroundings of home.

B. I get very restless if I stay around home for any length of time.

28. A. I like to dive off the high board.

B. I don't like the feeling I get standing on the high board (or I don't go near it at all).

29. A. I like to date members of the opposite sex who are physically exciting.

B. I like to date members of the opposite sex who share my values. 
30. A. Heavy drinking usually ruins a party because some people get loud and boisterous.

B. Keeping the drinks full is the key to a good party.

31. A. The worst social sin is to be rude.

B. The worst social sin is to be a bore.

32. A. A person should have considerable sexual experience before

marriage.

B. It's better if two married persons begin their sexual experience with one another.

33. A. Even if I had the money I would not care to associate with flighty persons like those in the "jet set".

B. I could conceive of myself seeking pleasure around the world with the "jet set".

34. A. I like people who are sharp and witty even if they do sometimes insult others.

B. I dislike people who have their fun at the expense of hurting the feelings of others.

35. A. There is altogether too much portrayal of sex in movies.

B. I enjoy watching many of the "sexy" scenes in movies.

36. A. I feel best after taking a couple of drinks.

B. Something is wrong with people who need liquor to feel good.

37. A. People should dress according to some standards of taste, neatness, and style.

B. People should dress in individual ways even if the effects are sometimes strange.

38. A. Sailing a long distance in small sailing crafts is foolhardy.

B. I would like to sail a long distance in a small but seaworthy sailing craft.

39. A. I have no patience with dull or boring persons.

B. I find something interesting in almost every person I talk with.

40. A. Skiing fast down a high mountain slope is a good way to end up on crutches.

B. I think I would enjoy the sensations of skiing very fast down a high mountain slope. 
Indicate how much you agree or disagree ( 4 = very strong agreement; $0=$ neither agreement nor disagreement; -4 = very strong disagreement) with each of the following statements. It is important that you respond to all items with only one choice. We are interested in how much you agree or disagree, not how others feel about these things or how one is supposed to feel about them. There are no right or wrong answers. Just give your first reaction.

1. I really enjoy a task that involves coming up with new solutions to problems.

2. I would prefer a task that is intellectual, difficult, and important to one that is somewhat important but does not require much thought.

3. I tend to set goals that can be accomplished only by expending considerable mental effort.

4. I am usually tempted to put more thought into a task than the job minimally requires.

5. Learning new ways to think doesn't excite me very much.

6. I am hesitant about making important decisions after thinking about them.

7. I usually end up deliberating about issues even when they do not affect me personally.

8. I prefer just to let things happen rather than try to understand why they turned out that way.

9. I have difficulty thinking in new and unfamiliar situations.

10. The idea of relying on thought to make my way to the top does not appeal to me.

11. The notion of thinking abstractly is not appealing to me.

12. I am an intellectual.

13. I only think as hard as I have to.

14. I don't reason well under pressure.

15. I like tasks that require little thought once I've learned them.

16. I prefer to think about small, daily projects to long-term ones.

17. I would rather do something that requires little thought than something that is sure to challenge my thinking abilities.

18. I find little satisfaction in deliberating hard and for long hours.

19. I more often talk with other people about the reasons for and possible solutions to international problems than about gossip or tidbits of what famous people are doing.

20. These days, I see little chance for performing well, even in "intellectual" jobs, unless one knows the right people. 
21. More often than not, more thinking just leads to more errors.

22. I don't like to have the responsibility of handling a situation that requires a lot of thinking.

23. I appreciate opportunities to discover the strengths and weaknesses of my own reasoning.

24. I feel relief rather than satisfaction after completing a task that required a lot of mental effort.

25. Thinking is not my idea of fun.

26. I try to anticipate and avoid situations where there is a likely chance I will have to think in depth about something.

27. I prefer watching educational to entertainment programs.

28. I think best when those around me are very intelligent.

29. I prefer my life to be filled with puzzles that I must solve.

30. I would prefer complex to simple problems.

31. Simply knowing the answer rather than understanding the reasons for the answer to a problem is fine with me.

32. It's enough for me that something gets the job done, I don't care how or why it works.

33. Ignorance is bliss.

34. I enjoy thinking about an issue even when the results of my thought will have no effect on the outcome of the issue. 
Directions: Which of the following do you personally favor or believe in ? Please circle YES or No. If you are absolutely uncertain, circle "?". How you feel about these things is what matters. It is not important how others feel, or how you think you are supposed to feel. There are no right or wrong answers. Just give your first reaction, and answer all items.

1. Death penalty

YES

No

2. evolution theory

YES

No

3. school uniforms

YES

No

4. striptease shows

YES

5. Sabbath observance

YES

6. hippies

YES

YES

YES

YES

YES

YES

NO

7. patriotism

8. modern art

9. self-denial

10. working mothers

11. horoscopes

12. birth control

YES

13. military drill

YES

14. co-ed dorms

YES

YES

YES

YES

17. integration

18. cousin marriage

YES

YES

YES

YES

21. chaperons

22. legalized abortion

YES

YES

YES

YES

YES

YES

?

No

No

NO

No

No

No

No

No

No

No

NO

No

No

No

No

No

No

No

23. foreign aid

No

24. student pranks

No

25. ABC laws

No

26. rap music

27. chastity

No

NO 


\begin{tabular}{|c|c|c|c|c|}
\hline 28 . & Nationalized health & YES & $?$ & No \\
\hline 29. & "old money" & YES & $?$ & No \\
\hline 30 . & female judges & YES & $?$ & No \\
\hline 31. & conventional clothing & YES & $?$ & No \\
\hline 32 . & drinking age & YES & $?$ & No \\
\hline 33. & apartheid & YES & $?$ & NO \\
\hline 34. & nudist camps & YES & $?$ & No \\
\hline 35 . & church authority & YES & $?$ & No \\
\hline 36. & disarmament & YES & $?$ & No \\
\hline 37. & censorship & YES & $?$ & No \\
\hline 38. & white lies & YES & $?$ & No \\
\hline 39 . & corporal punishment & YES & $?$ & No \\
\hline 40 & mixed marriage & YES & $?$ & No \\
\hline 41 . & strict rules & YES & $?$ & No \\
\hline 42 . & progressive music & YES & $?$ & No \\
\hline 43. & euthanasia & YES & $?$ & No \\
\hline 44. & living together & YES & $?$ & No \\
\hline 45 . & language requirement & YES & $?$ & No \\
\hline 46. & divorce & YES & ? & NO \\
\hline 47. & inborn conscience & YES & $?$ & No \\
\hline 48 . & illegal aliens & YES & ? & No \\
\hline 49 . & Bible truth & YES & ? & No \\
\hline 50 . & fraternity parties & YES & ? & No \\
\hline
\end{tabular}


1.

A writer sent his latest novel to a publisher. At the meeting the publisher said: "Your novel is excellent, but we only take work from authors with well-known names." "Wonderful", beamed the writer, "my name is Smith."
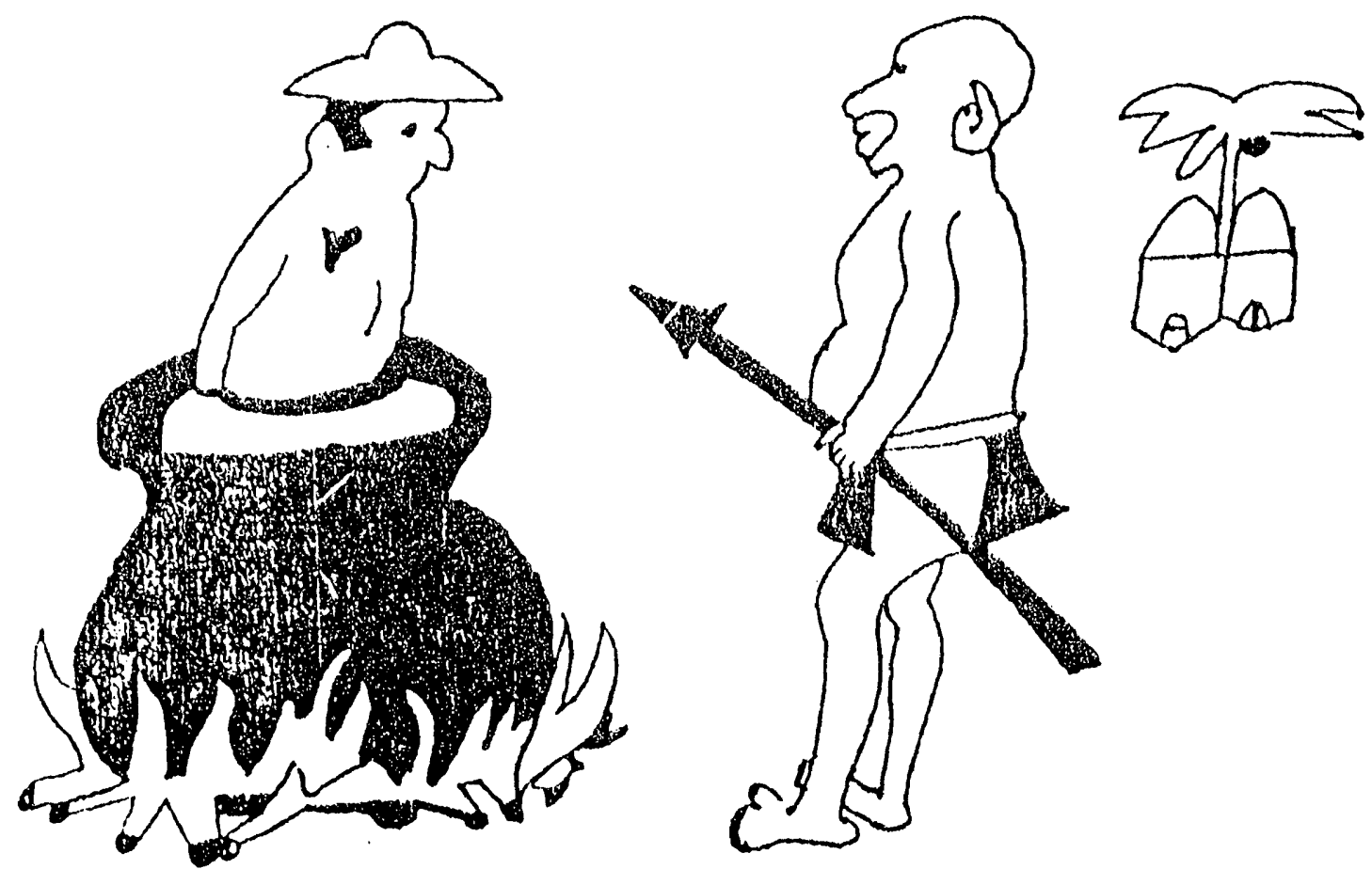

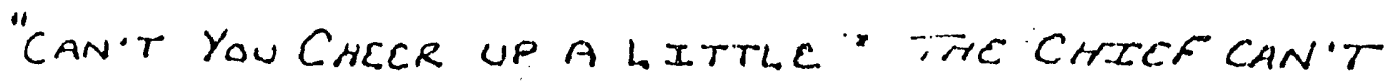
STANO SAD OINNCRS!" 

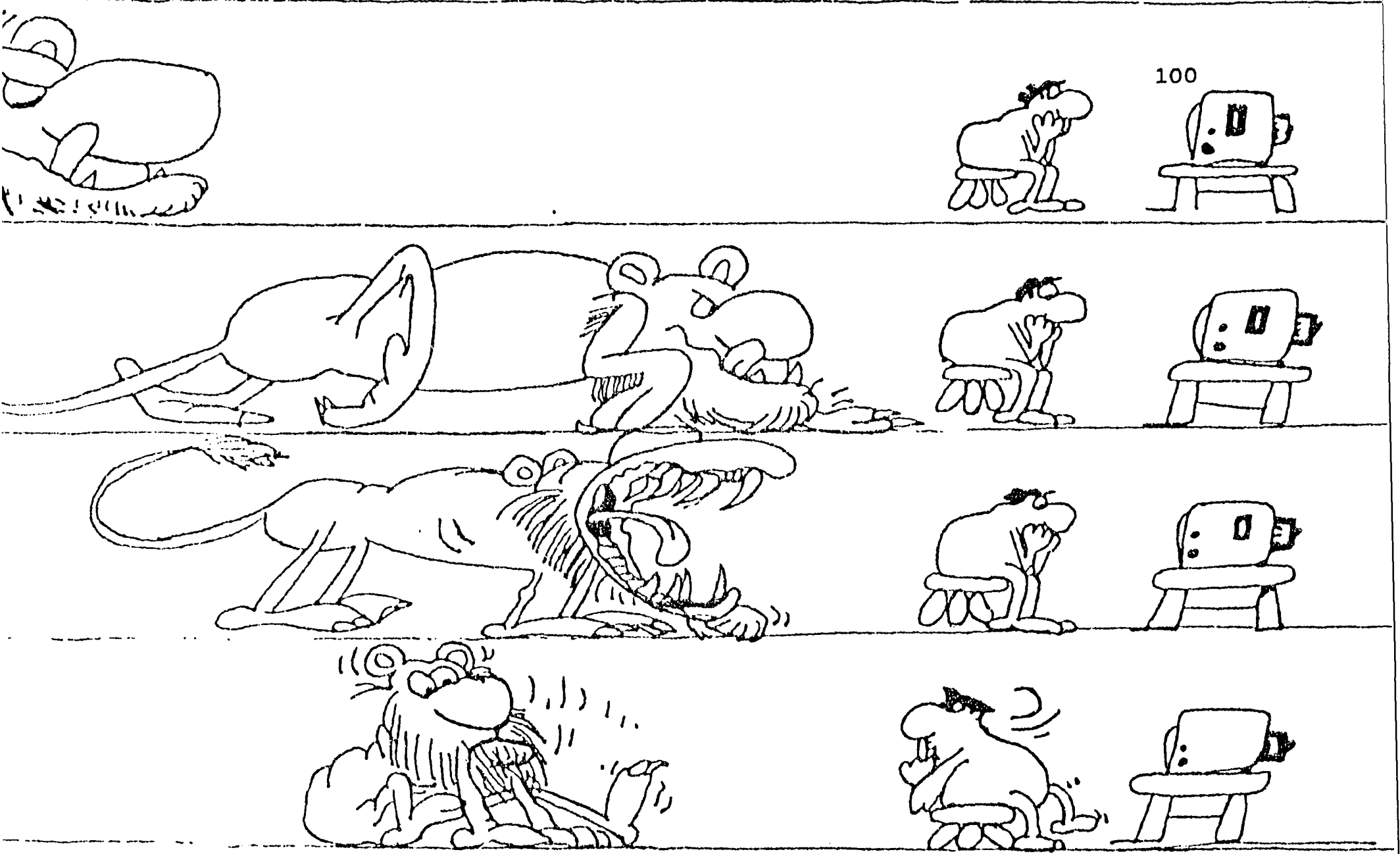

4. "Why don't you want to get married again, Ms. Thompson?" "Well you know having my animals is like having a man: the dog growls all day, the tomcat is out all night, and the parrot talks abusively from morning til dark." 
5.
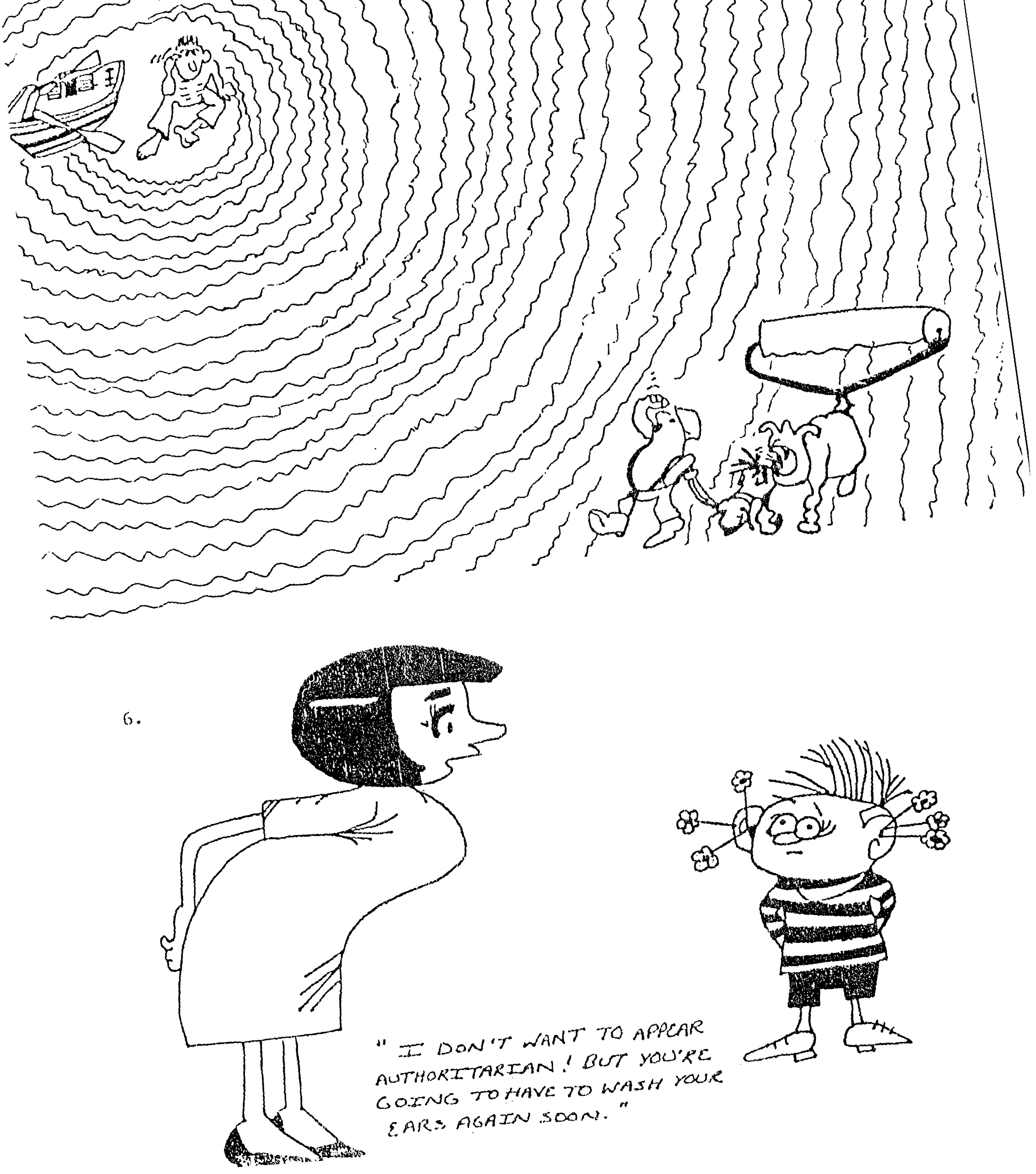

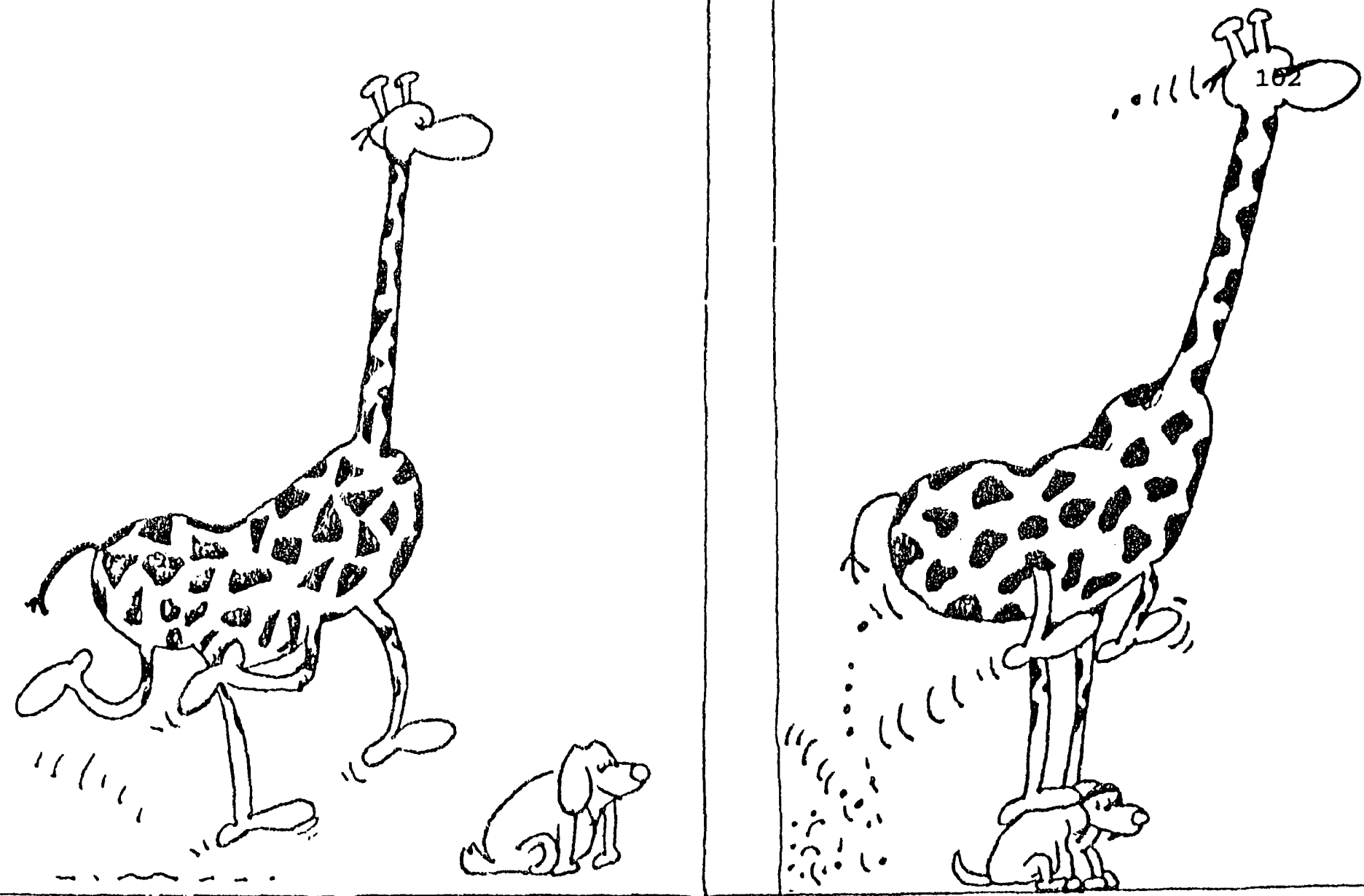

At midnight, Harry meets a skeleton at the cemetery. It 8. has a gravestone under its arm. "What are you doing with that gravestone?" asks Harry, puzzled. The skeleton: "No one gets out of here without papers." 
9. A lady repeatedly dropped hints to her husband about wanting a fur coat. Finally, he gave in and bought her a fur jacket. The woman was overjoyed, but suddenly became pensive and said: "The jacket is really wonderful, but actually any creature that gets skinned for a coat like this should be pitied." "Thank you for your sympathy," said the man.

10 .

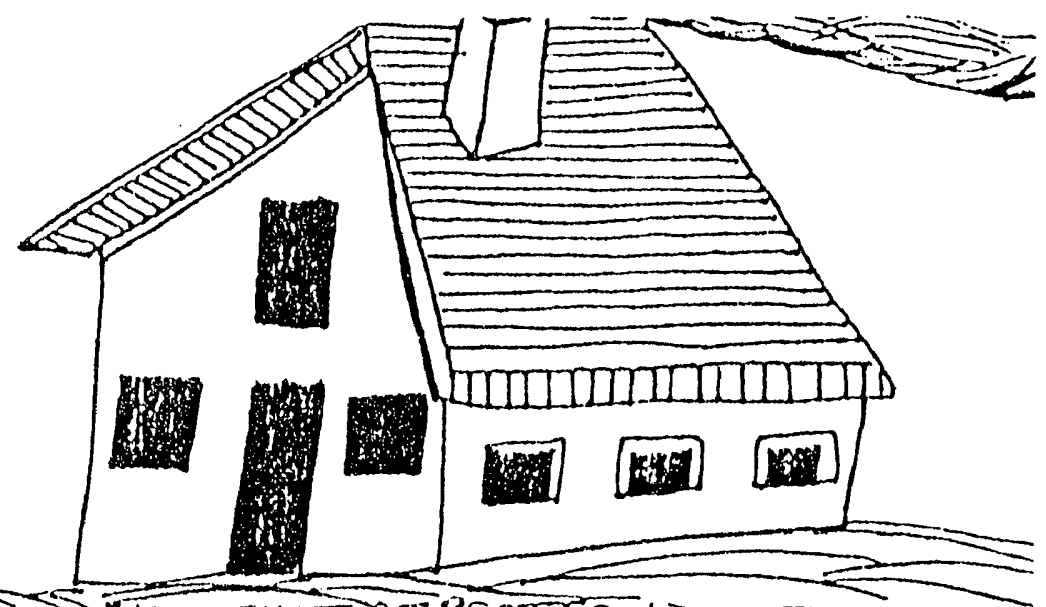

"YOU KNOW WIH THE"WIND ALWAYS COMES FROM THAT DIRECTION? BECHUSE ERSLES NEKCA RLY CIACKLLAROS.

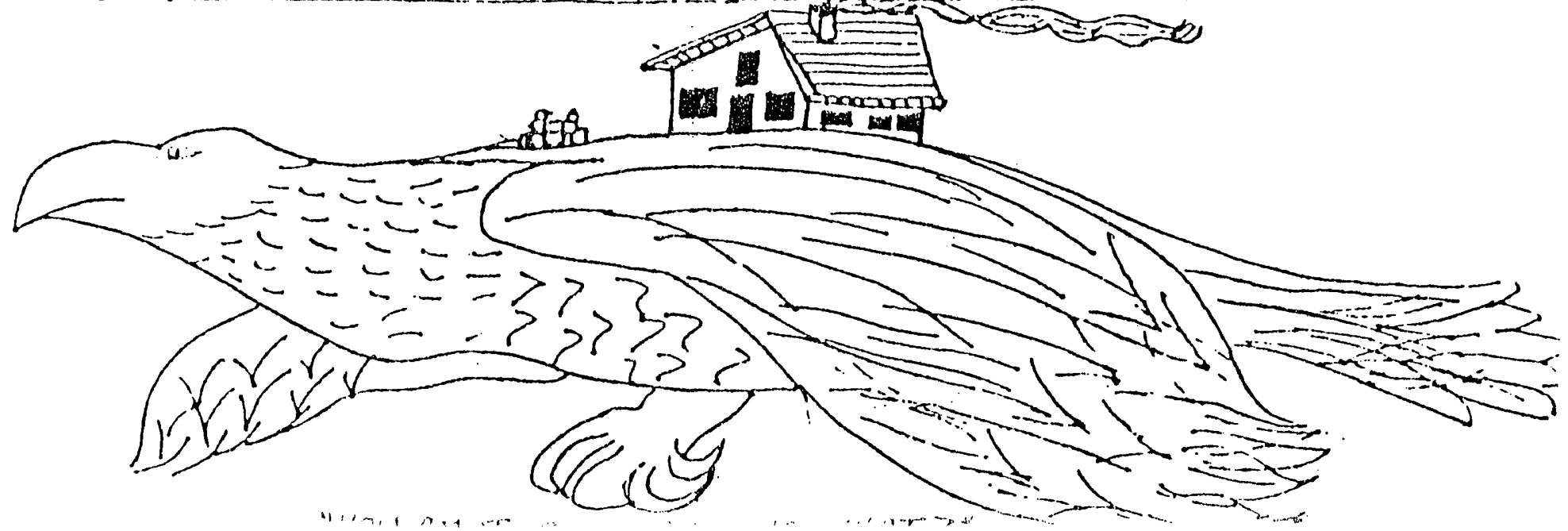




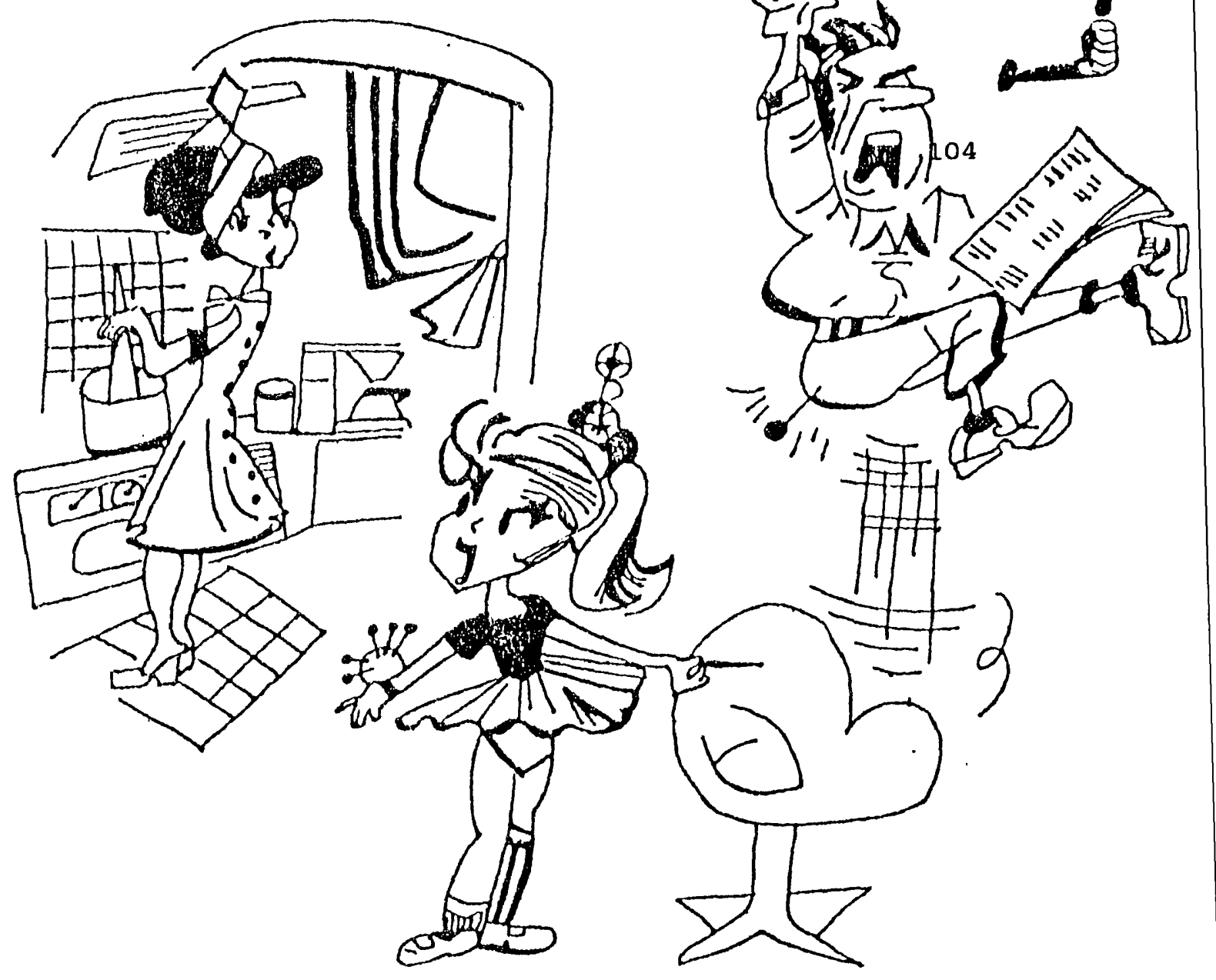

"I"," USING ACUPUNCTURE TO HELP DADDY STOO SMOKING!!"

12.

SAY, DOW'r z"

KNOW rou??

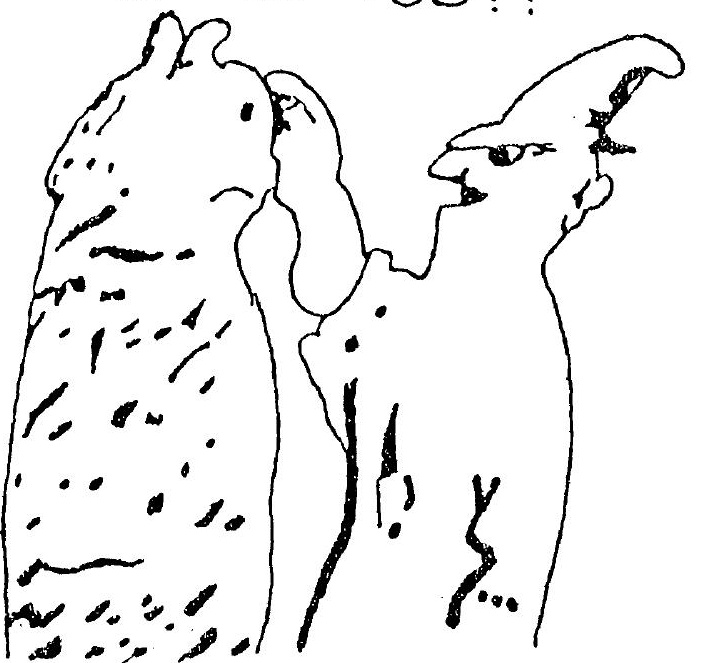

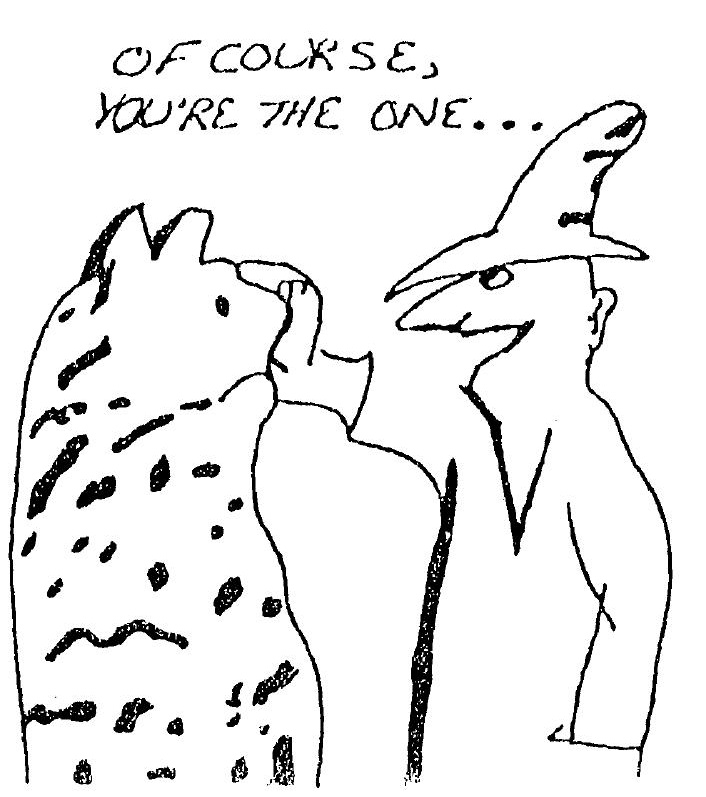

... WITHTHE

SNOUT PEOPLE

ALWAYS PKESS!

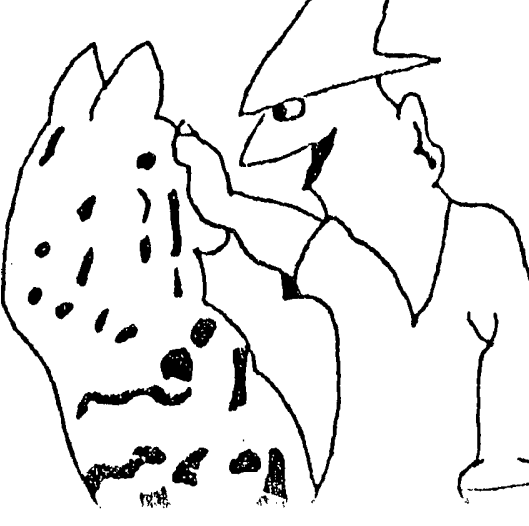


13. One day the lion was running through the desert and ran into at monkey. "Who is the king of the jungle?" "You are", said the monkey. "Well, that's a good answer, I'll let you live." The lion ther met an antelope and a giraffe who gave the same answer. Finally, he asked an elephant: "Who is the king of the jungle?" The elephant picked up the lion with his trunk, threw him in the air and lifted one leg menacingly. The lion responded: "Boy, you can't even ask a question..."

14.

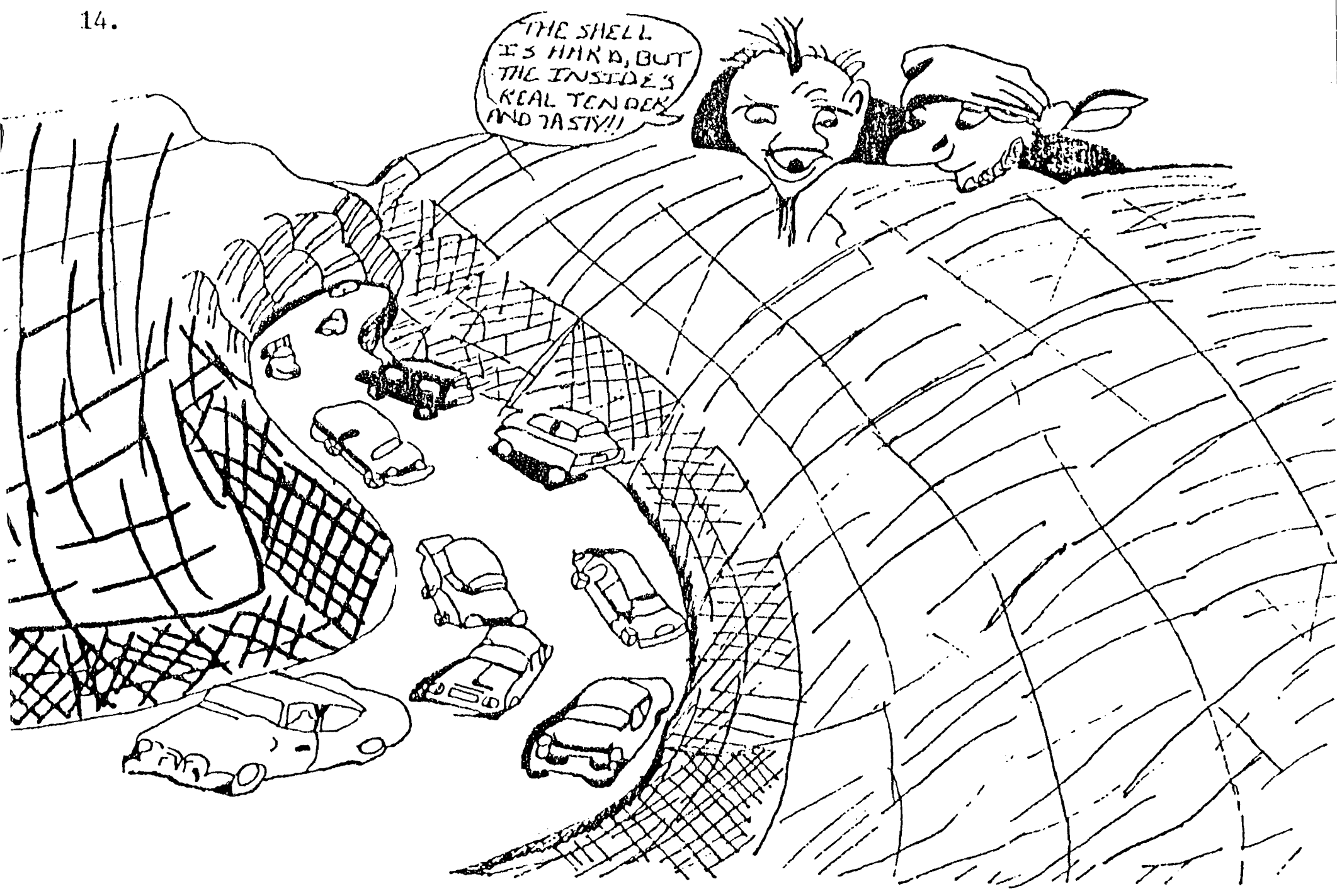


15.
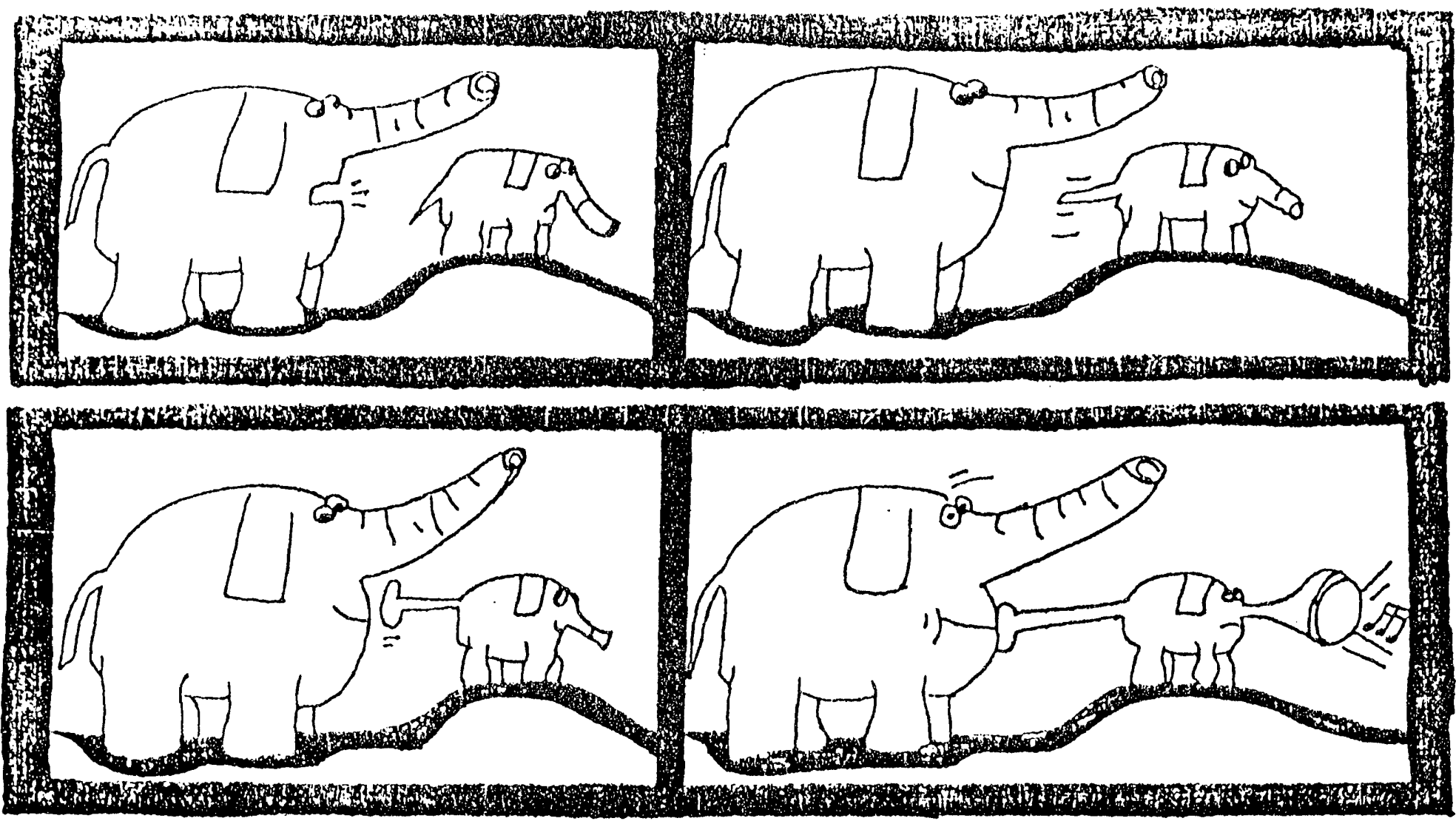

16. Wife: "There's a man at the door collecting money for the new swimming pool. What shall I give him?" Husband: "Three buckets of water." 
17.
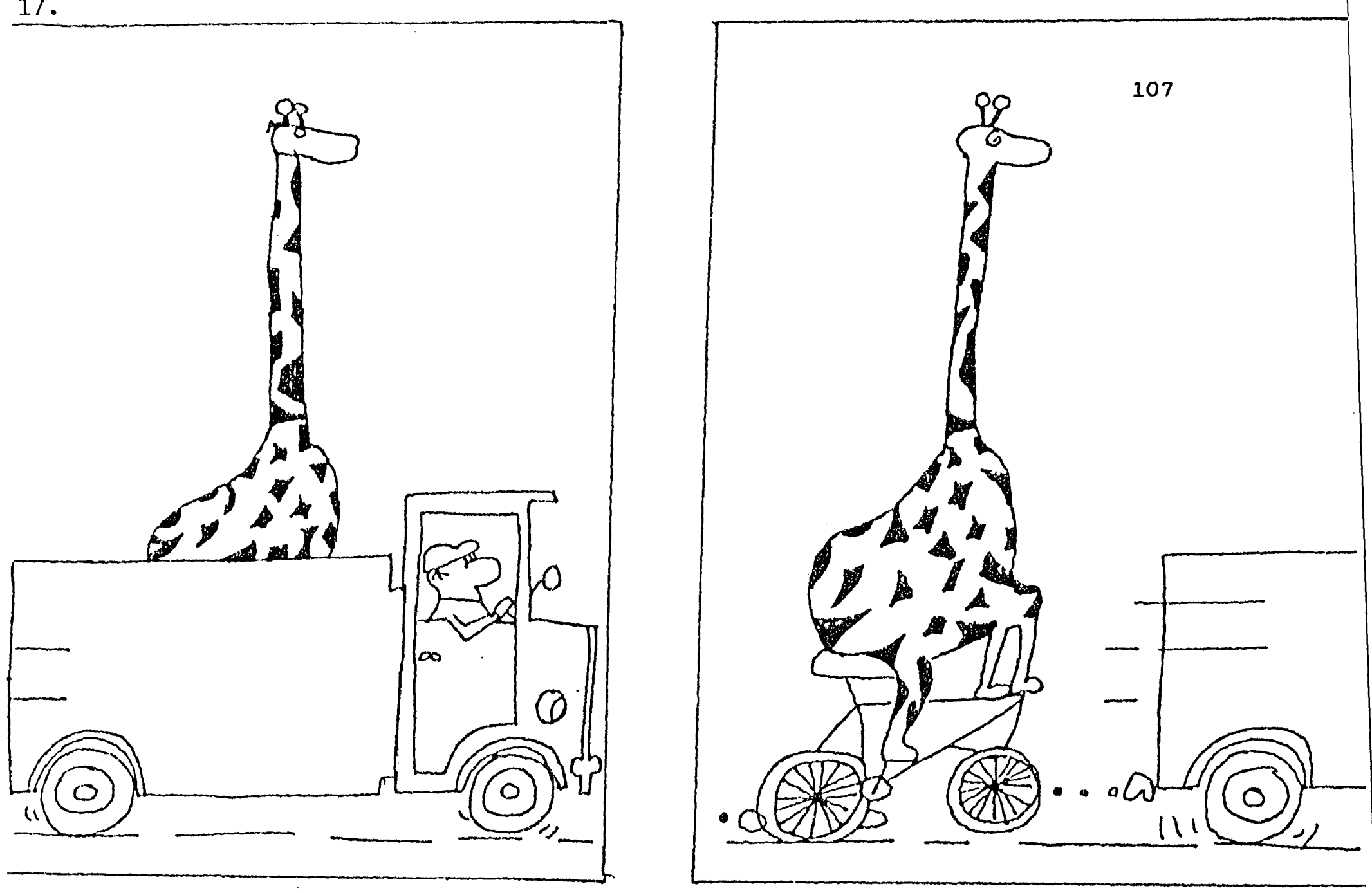

18.

JOKE OF THE MONTH

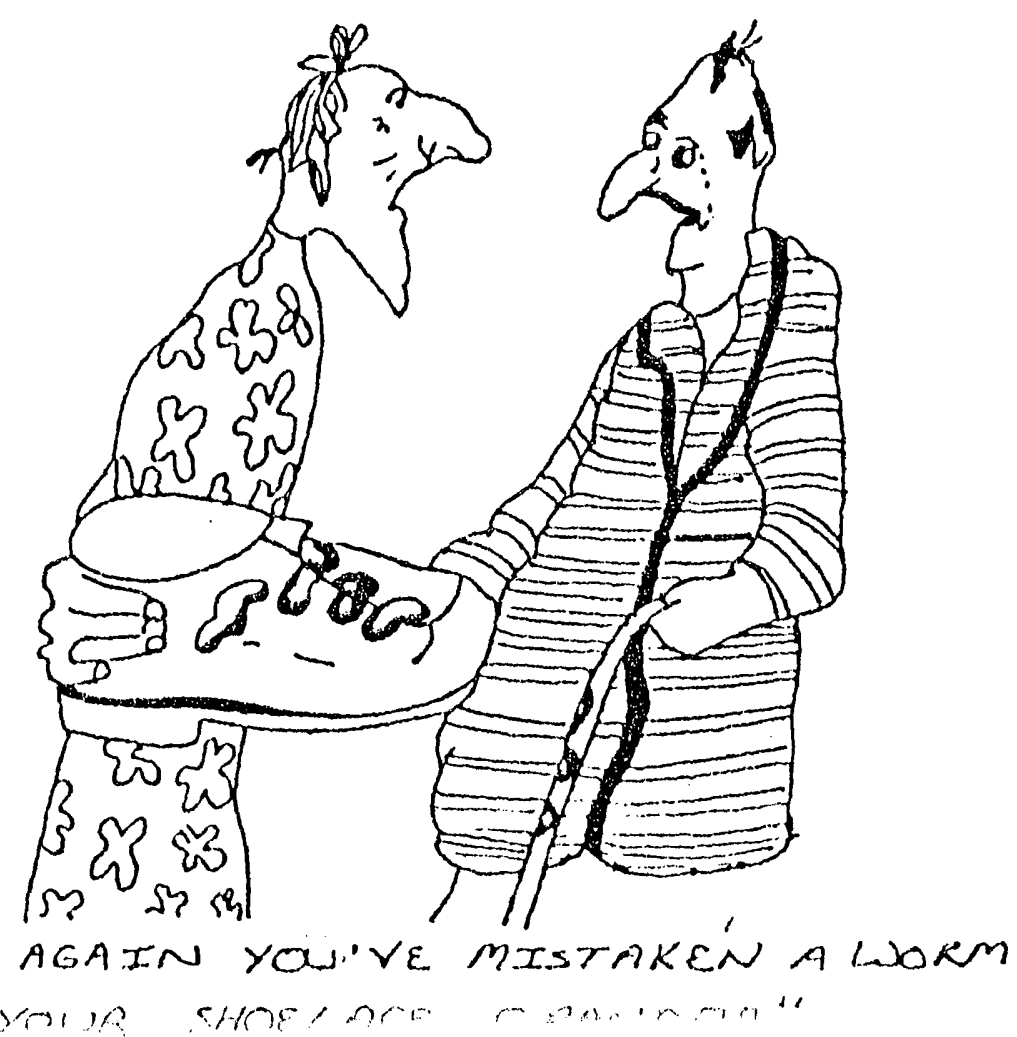


19. The young dancer was on her way to audition for a job with the fanous director. She excitedly paced back and forth at the bus stop. Periodically, she practiced her routine of short little steps. A little boy nudged her elbow and whispered: "Come with me lady, I'1I show you where it is."

20.

7. $R Y B A$

\section{YY + SAUYON}

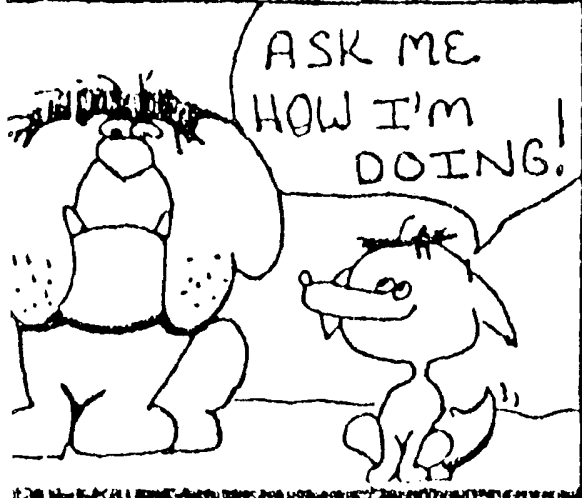

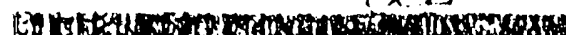

EASE ASK ME HOW

$\because M$ DarNG, JUST

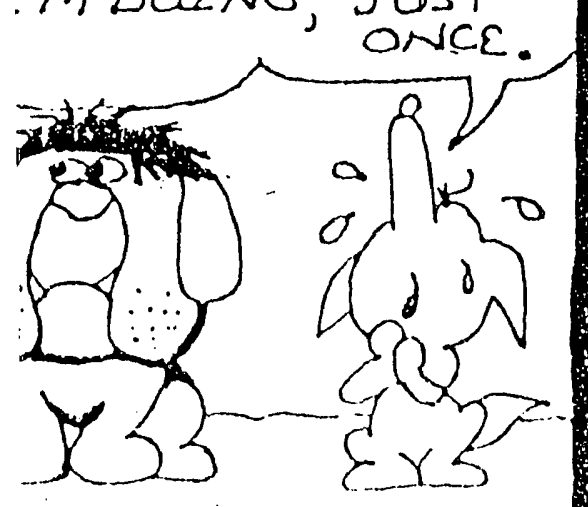

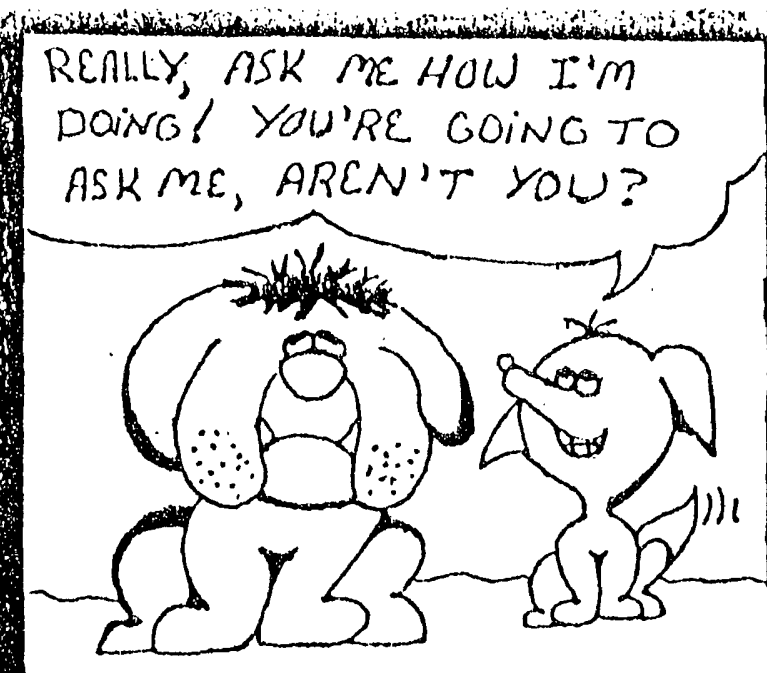

ALL RIGHTI! HOW'RE YOU DOING?)

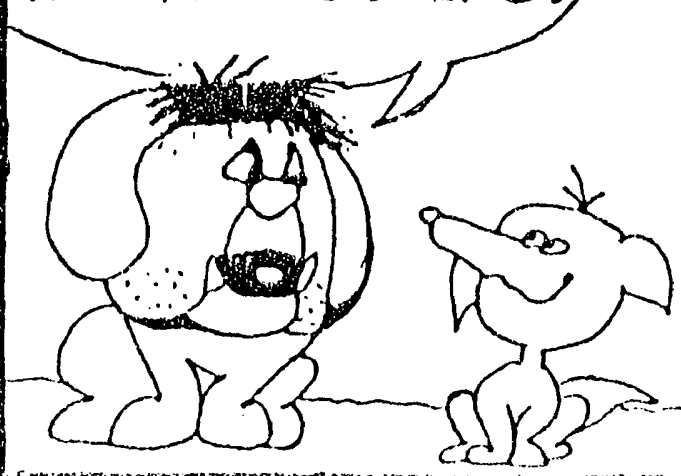

ASK ME HOW I'M DOING. . NOW.
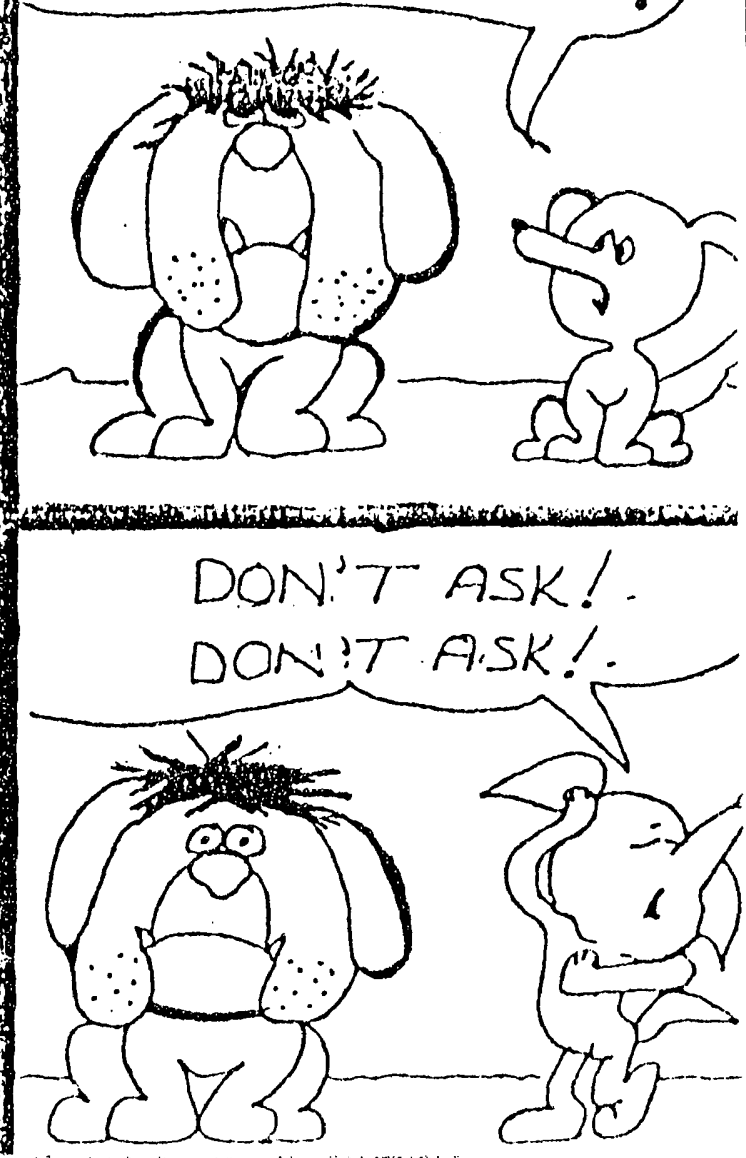


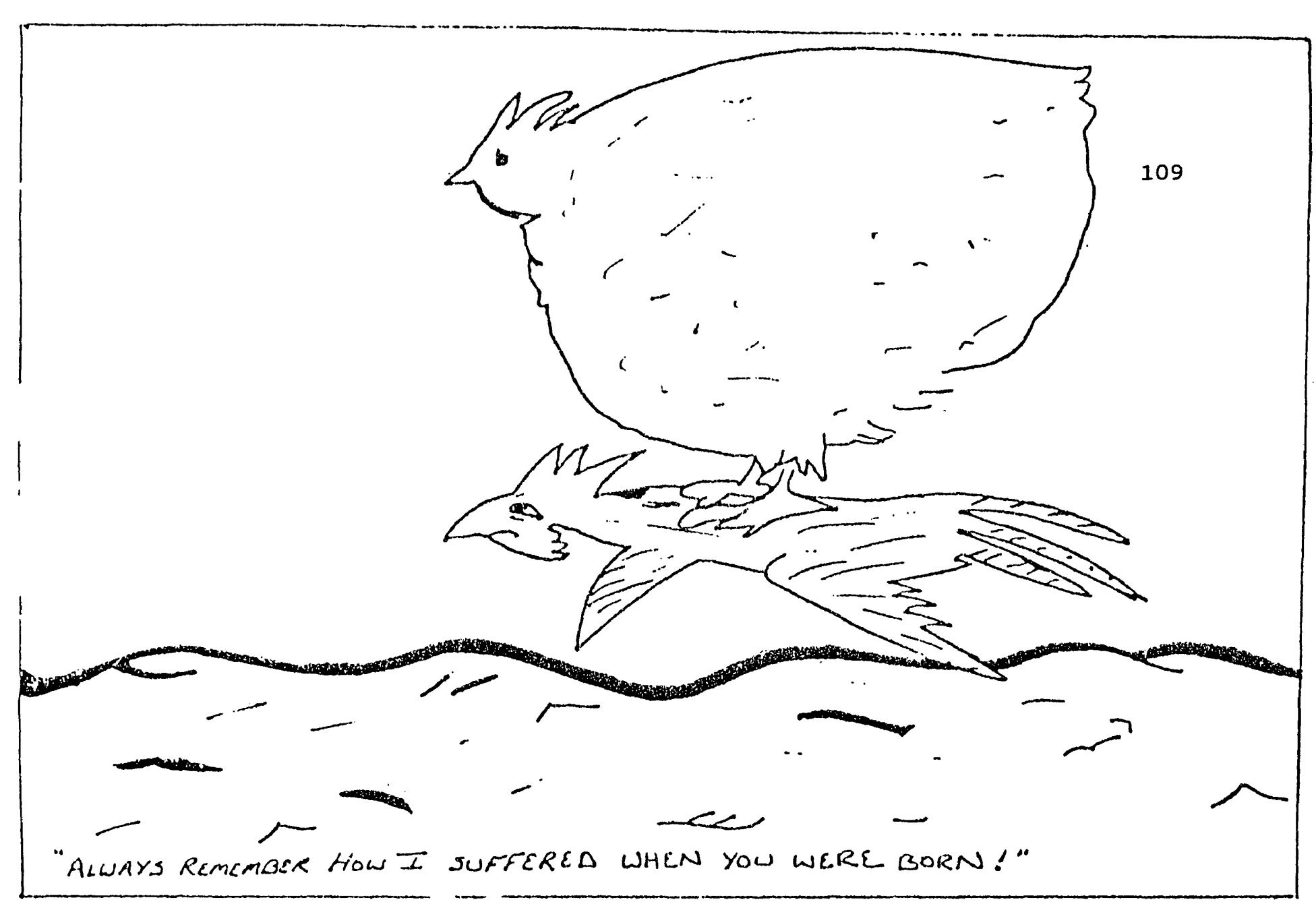

Work, WORK...

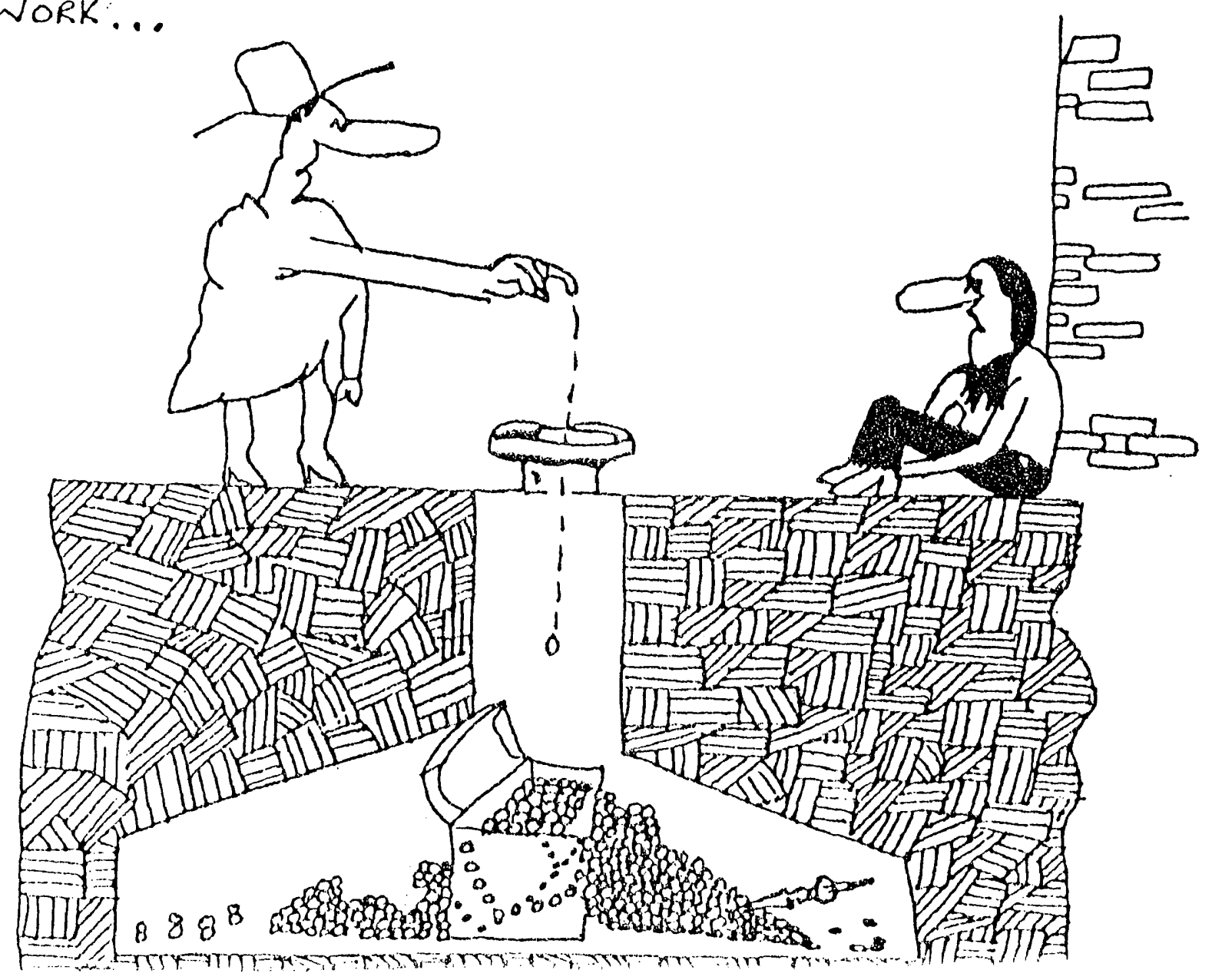


23.

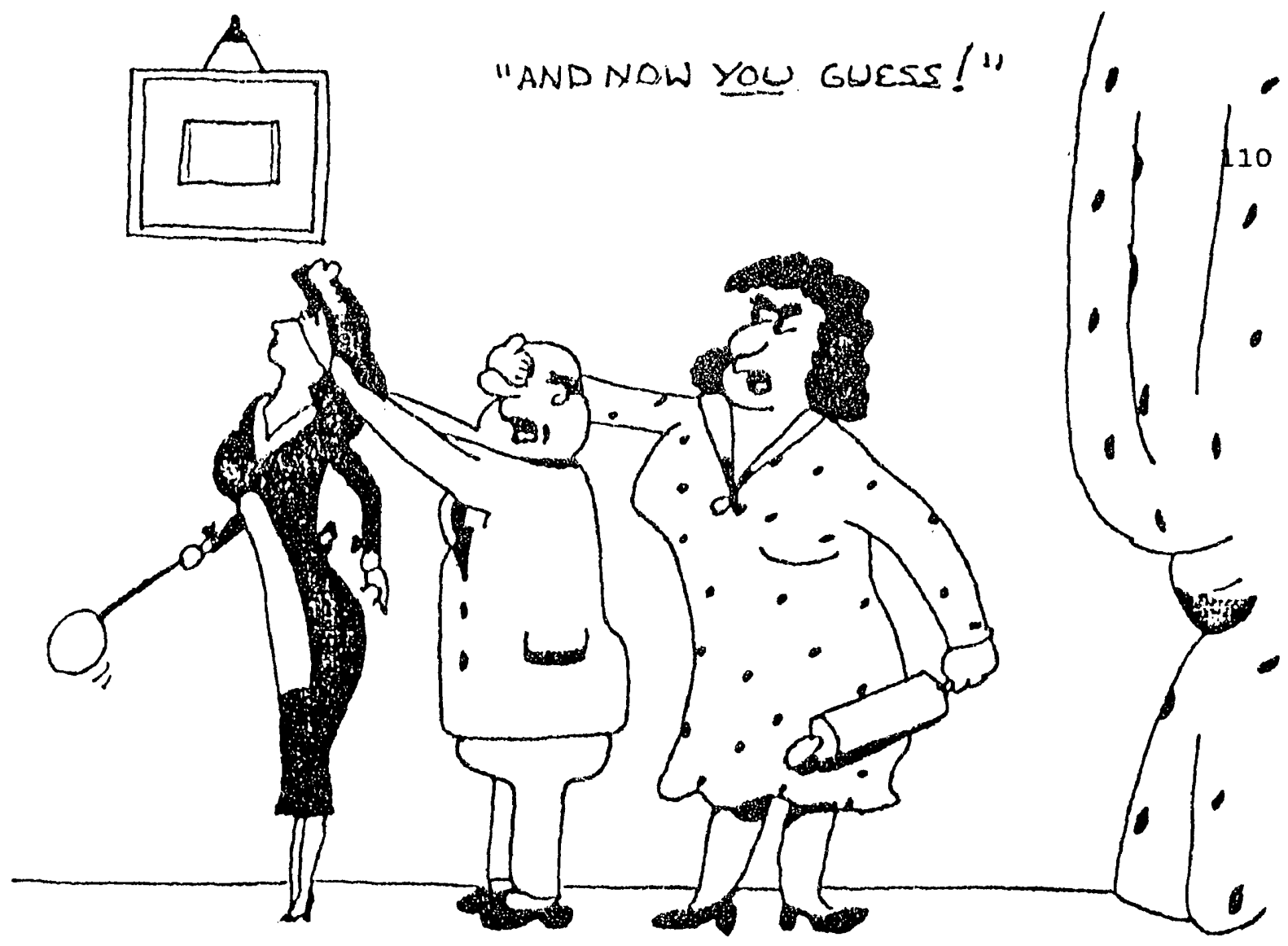

24.

$T$ FREDDY'S

2

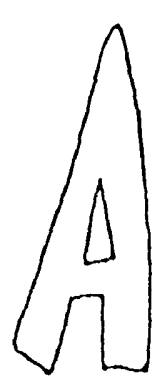

3ERT SHELDON
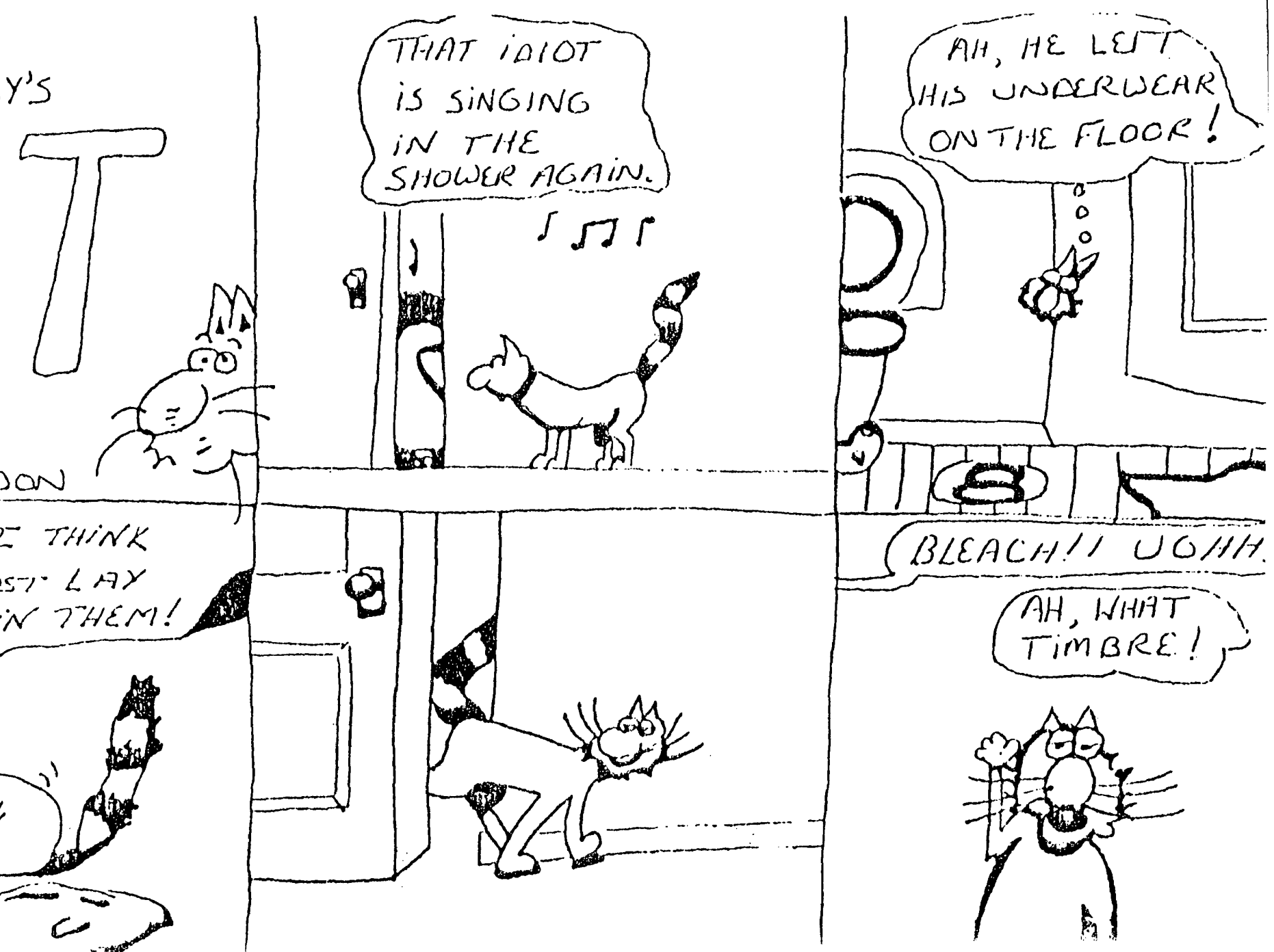

$W E L L, Z$ Z THINK I'L JULST LAY AN EGG IN THEN! :

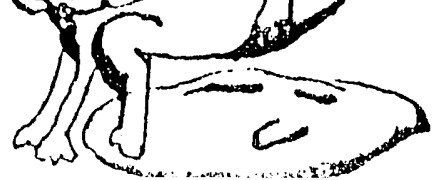

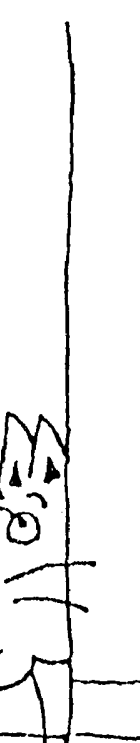

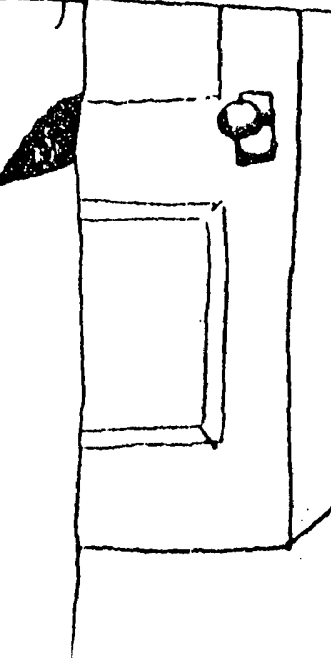

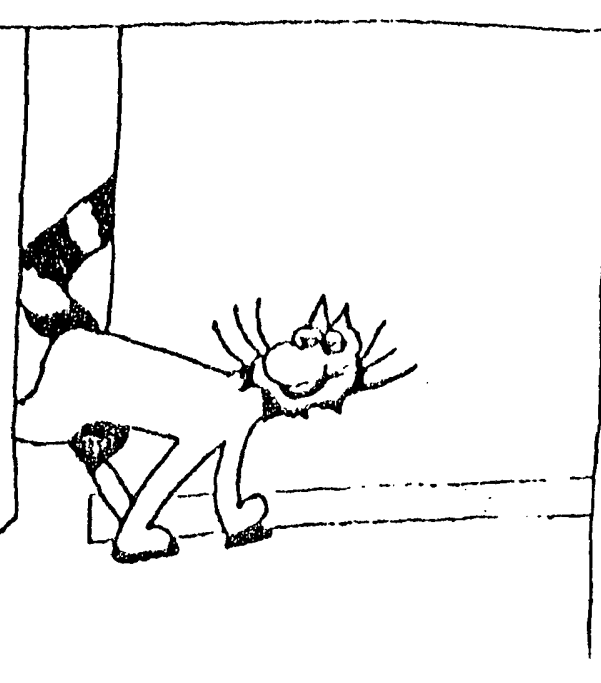


qui

25.

111

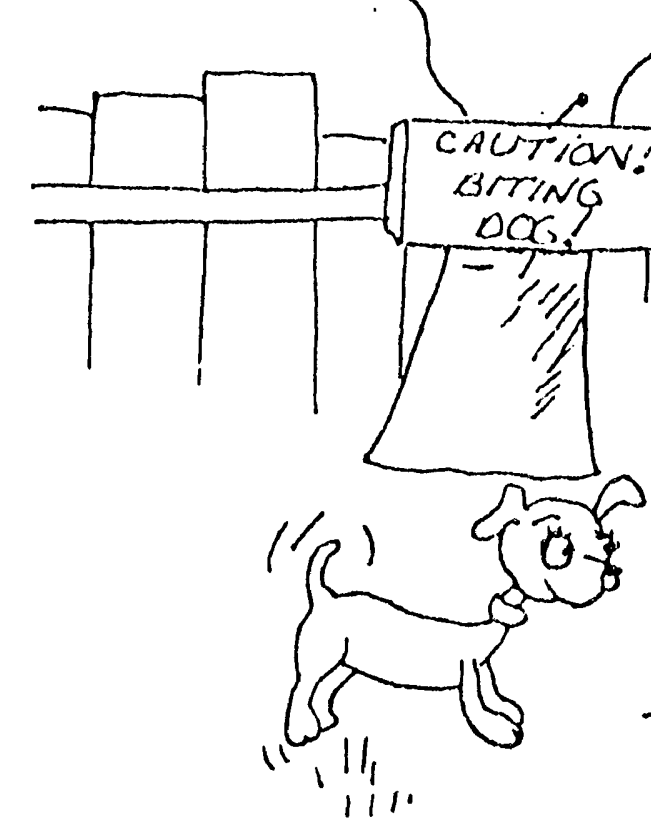

26.
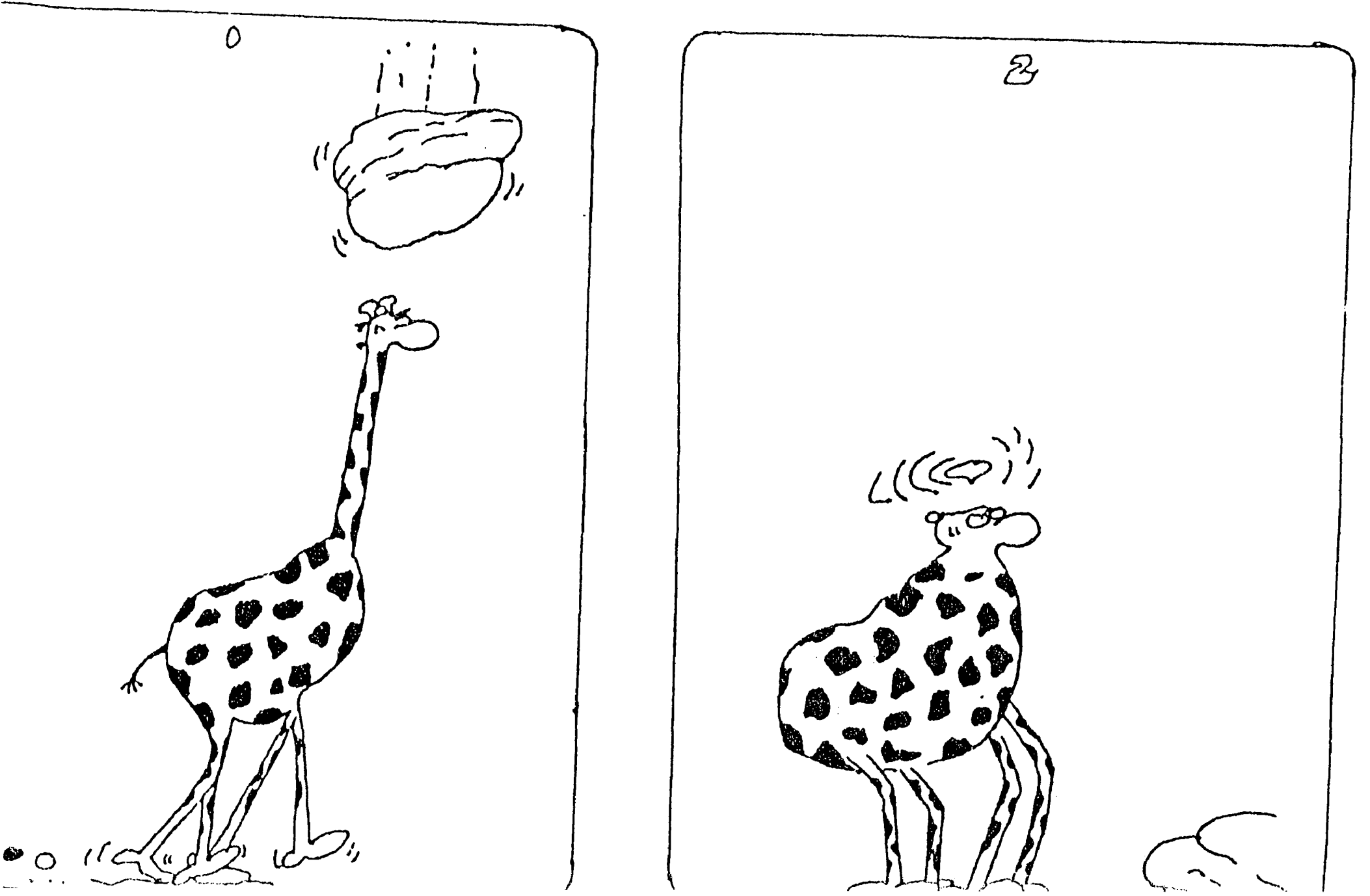
27. "Daddy", asked little David, "what's a celebrity?" "Well son, its a person who does something exceptional in order to become well known, and then once he becomes known he wears dark glasses so he won't be recognized."

28.

AT PRED Y Y

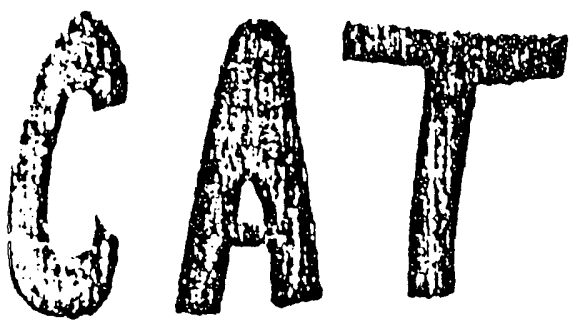

BERT SHELDON;

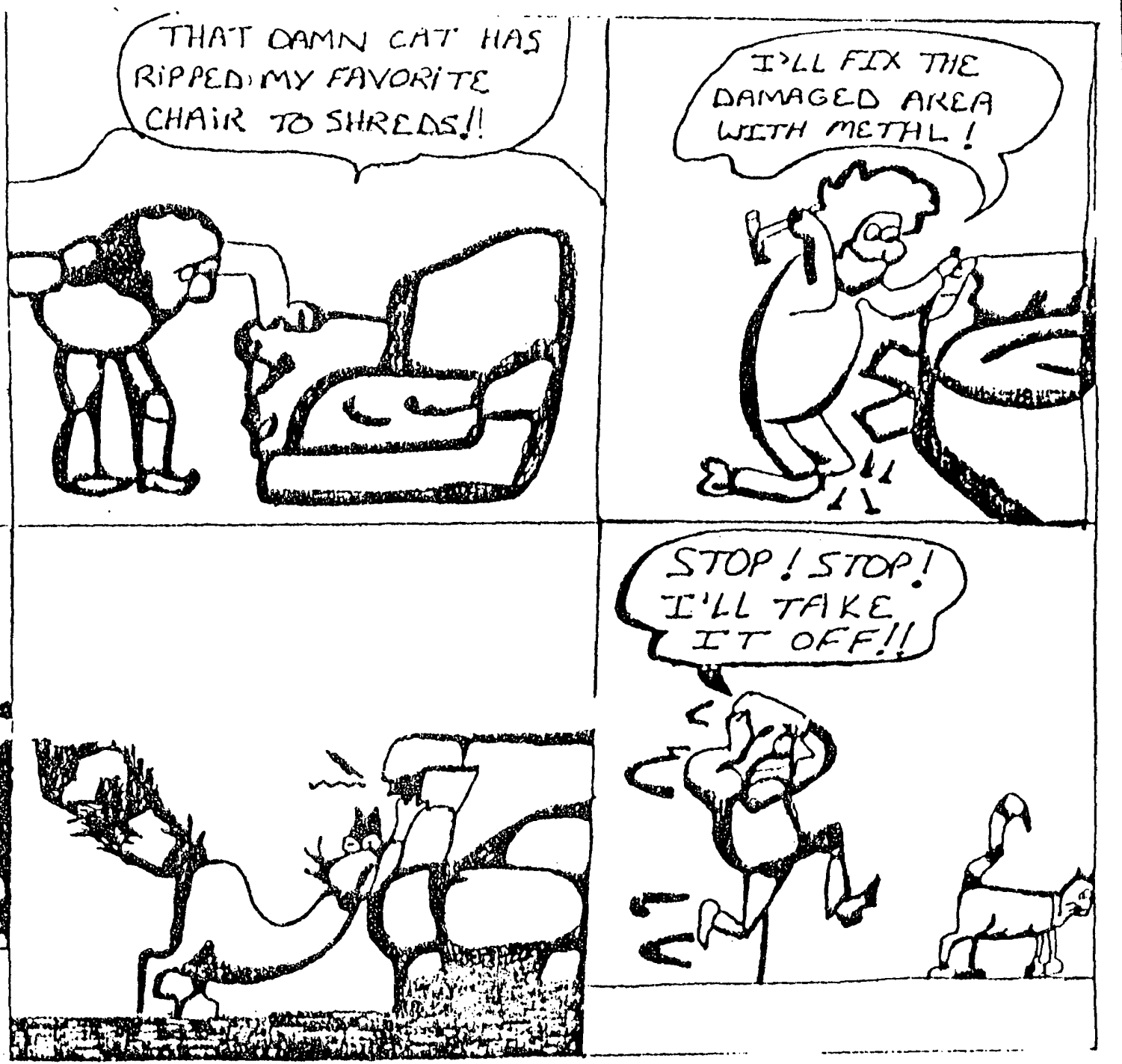


29.

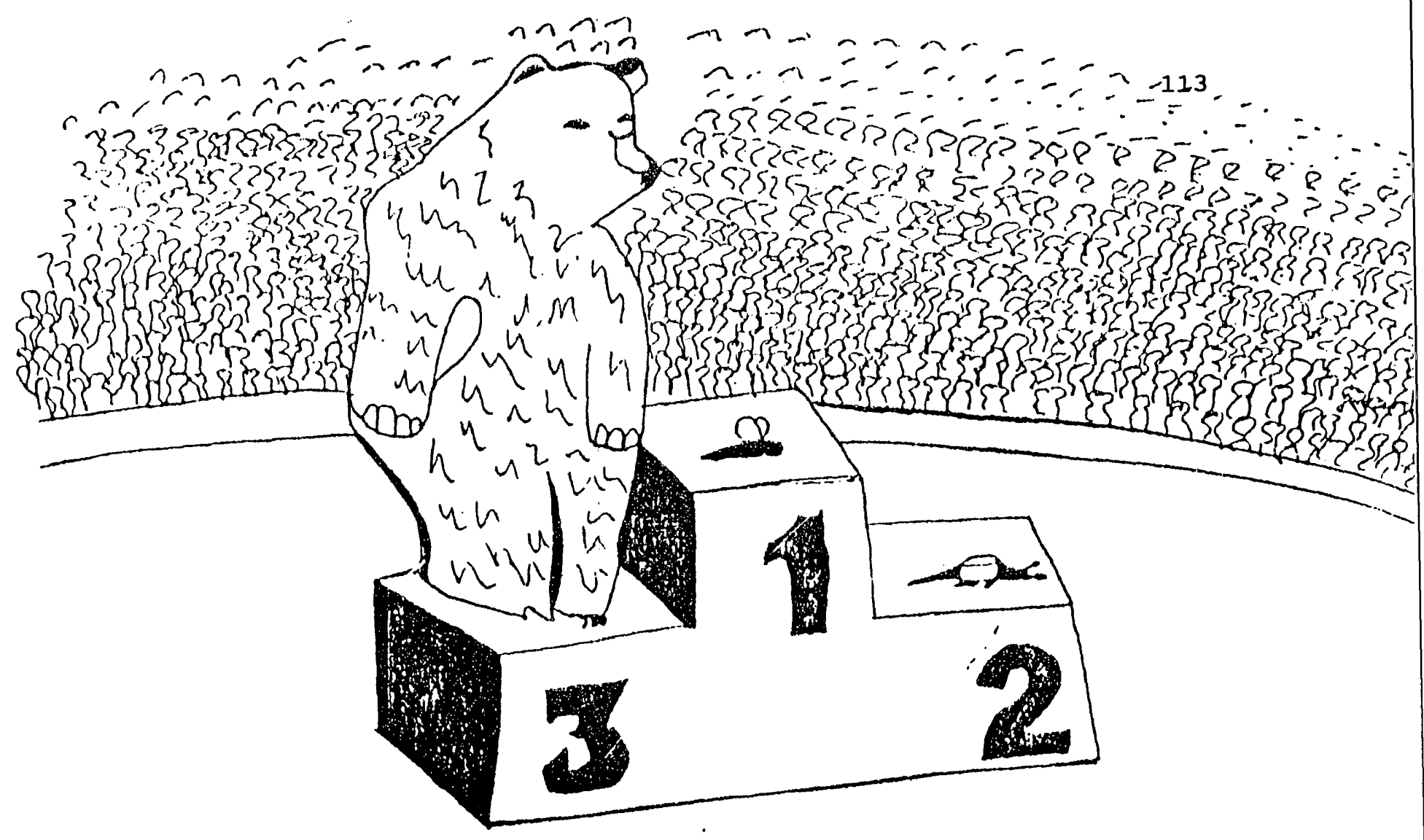

ACTUALLY, I SHOULD BE GLAD-THIRD BEST IN' BUZZING-BUT STILL.

30. The telegram delivery boy rings the doorbell at Professor Duffey's house. "Here's a telegram for you Professor!" "Thank you", nodded the Professor in his typical friendly but absent-minded way, "I don't need any today," and he was quickly back at his work. 
31. "Do you believe in the Easter Bumy?" Margle asked her new fricend in kindergarten, "Why not?", he answered. "With today's technology, anything is possible."

32.

Gut gegeben
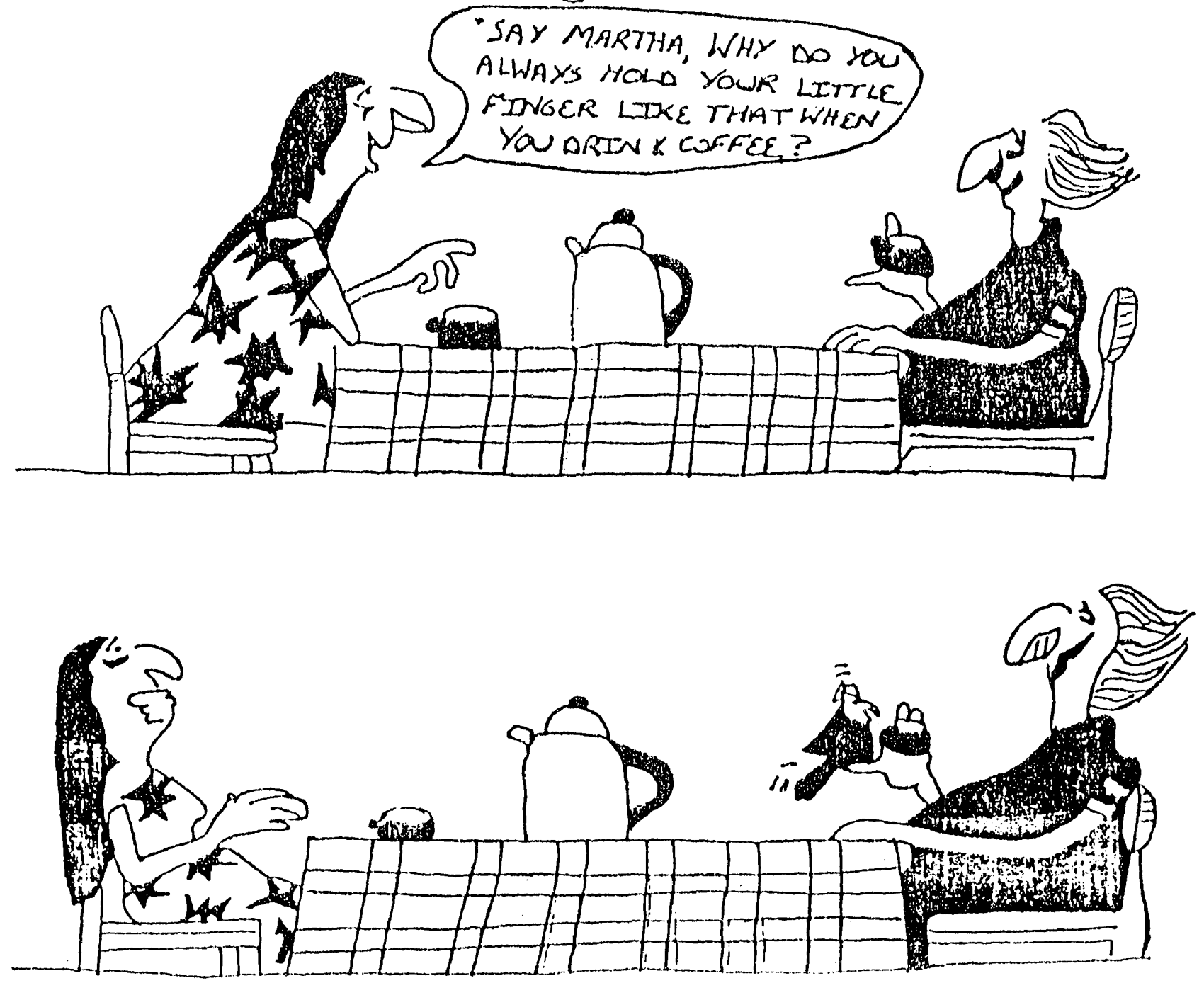
33.

WHAT DILD YOU JUST

COMPREE ME TO?

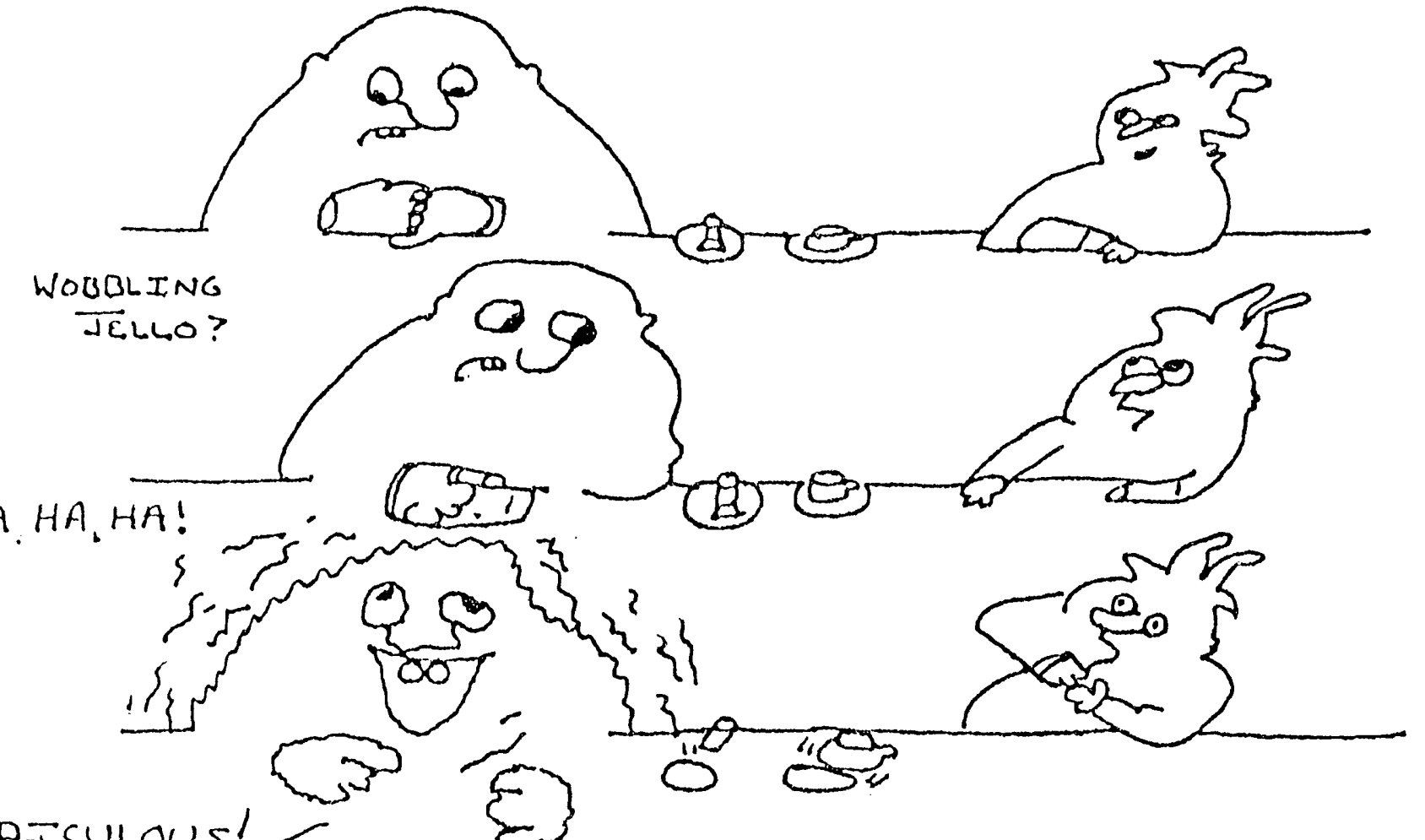

HA. HA. HA!

Daculous! \%

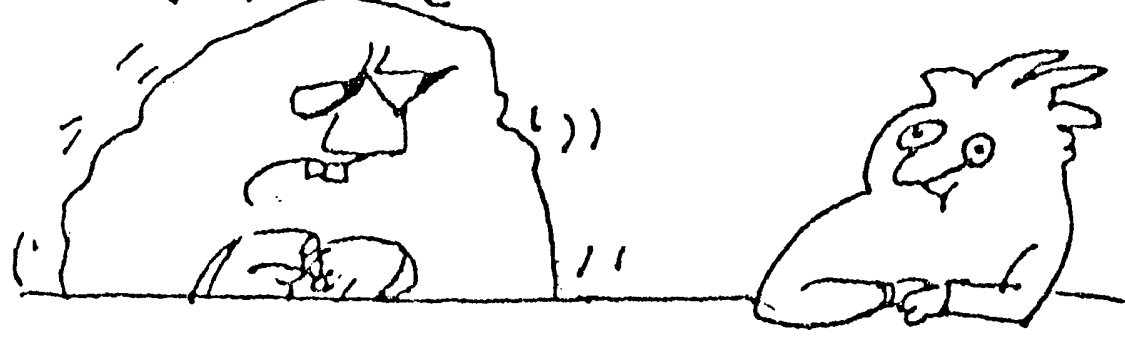

34. "Waiter, what are these little animals jumping up and down in my salad?" asked the lady in the restaurant. The waiter replied with his quick wit: "Dear lady, surely you have heard of vitamins." 
35. The absent-minded professor finished his meal, paid the bill and left his table. Two minutes later, he returned to the table and asked to see the menu. "But you've just eaten," said the waiter. "Ah," the professor blurted out, "Those blasted revolving doors."

36.
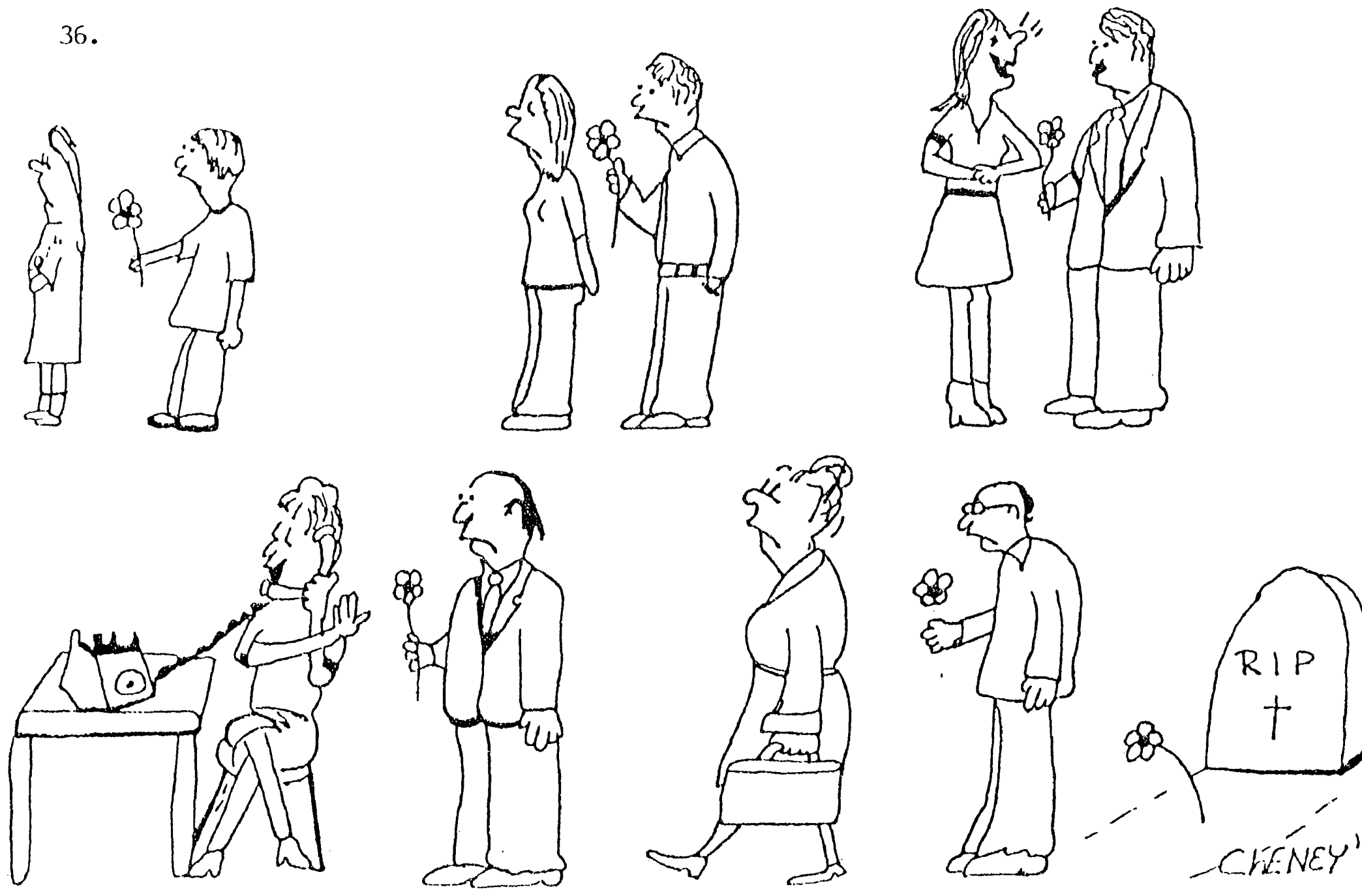


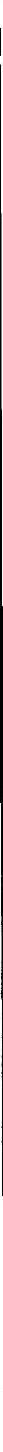




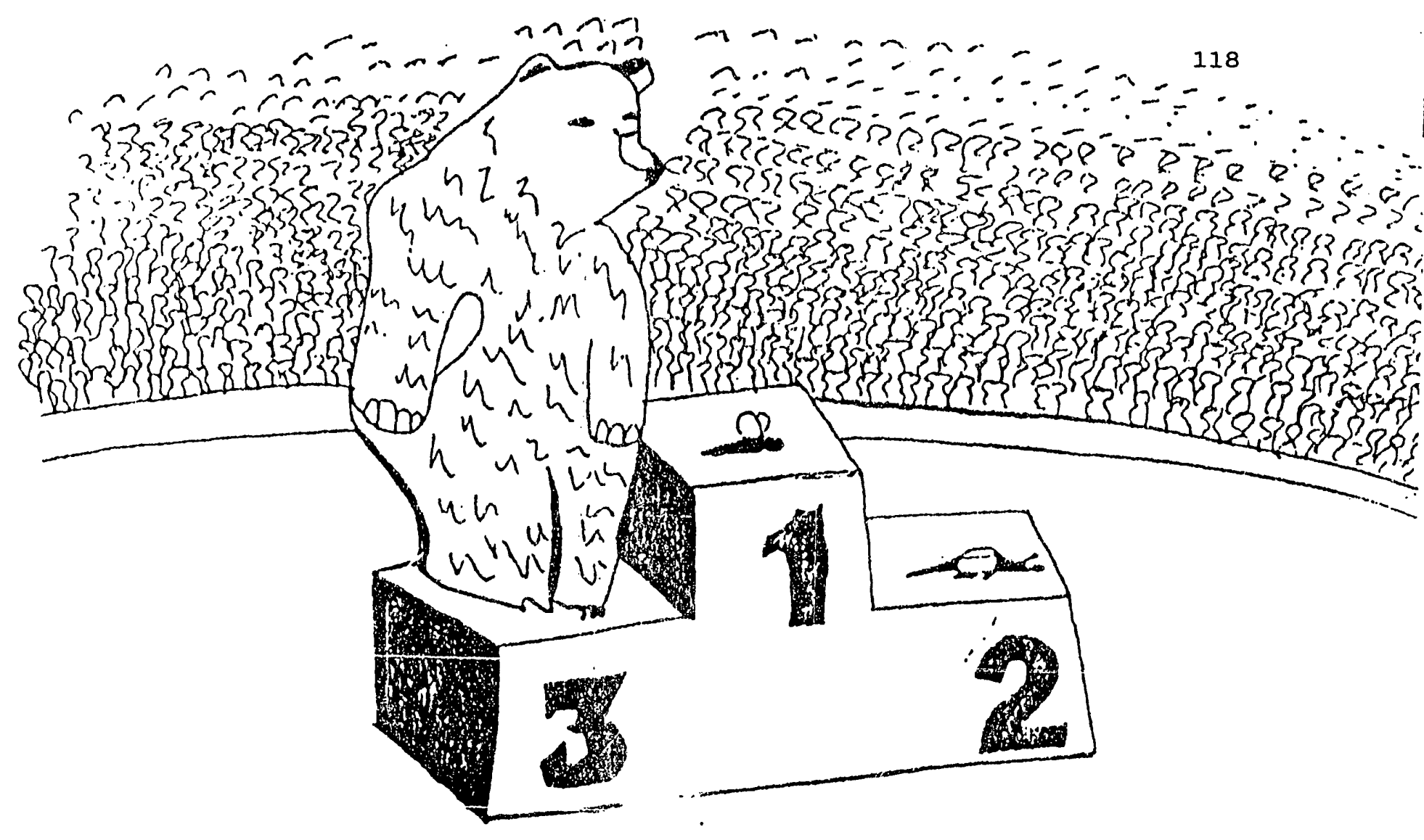

ACTUALLY, I SHOULD BE GLAD- THIRD BEST IN' BUZZING - BUT STILLL.

FUNNINESS $=$

AVERSIVENESS $=$

BECAUSE. . 
B.

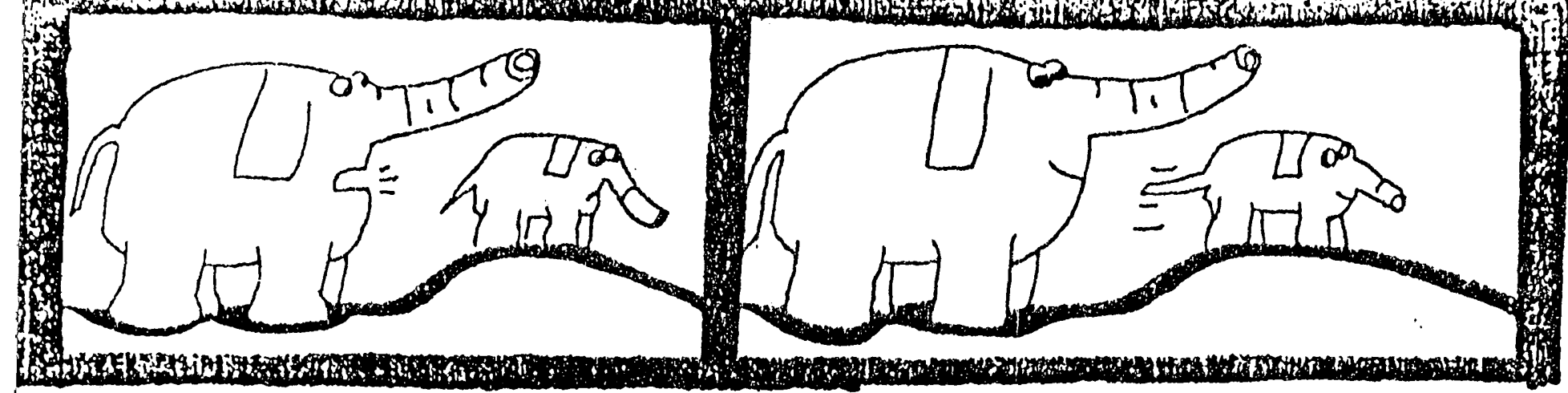
(2)

JNNINESS $=$

VERSIVENESS =

ECAUSE. . . 


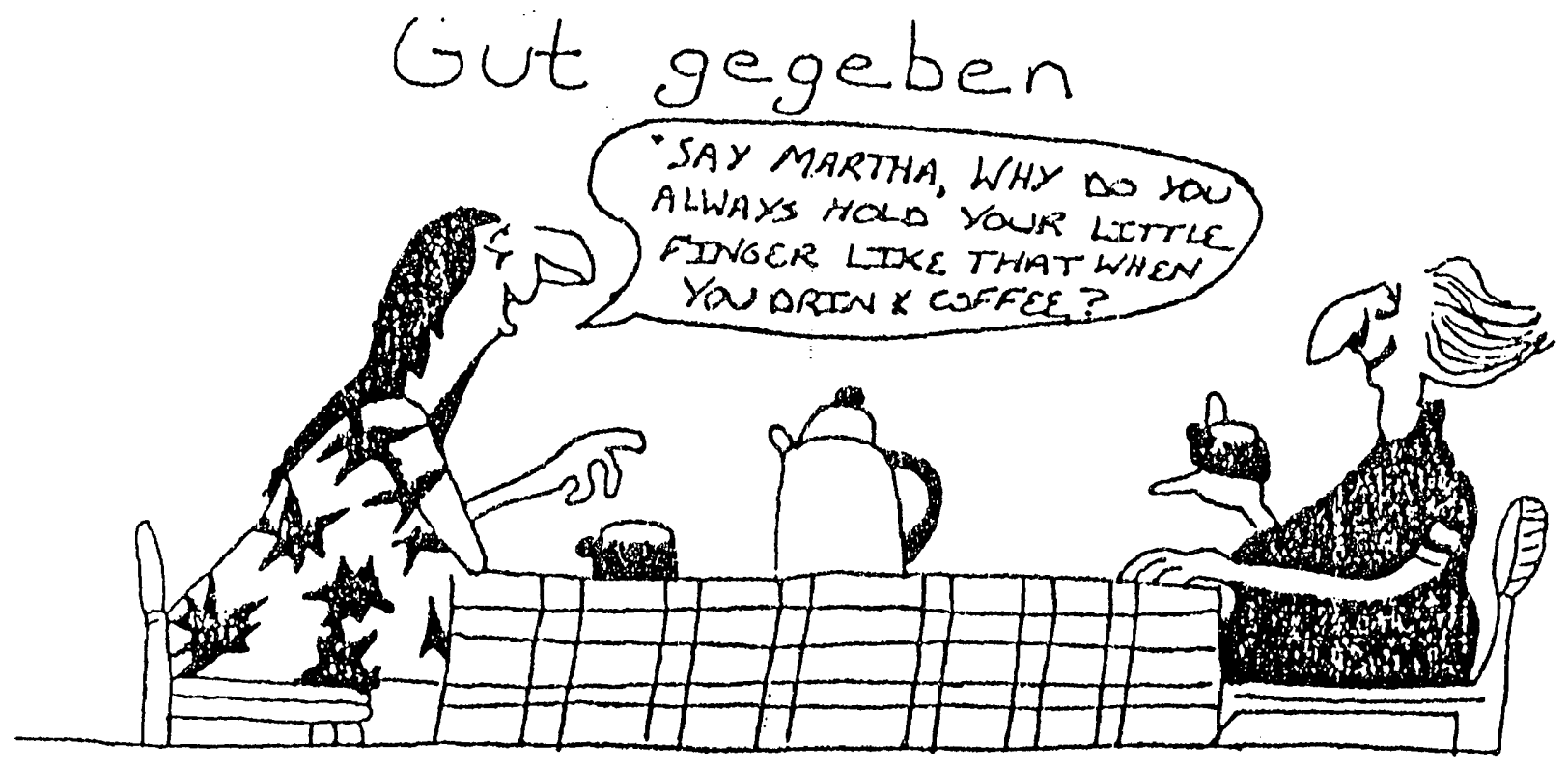

120

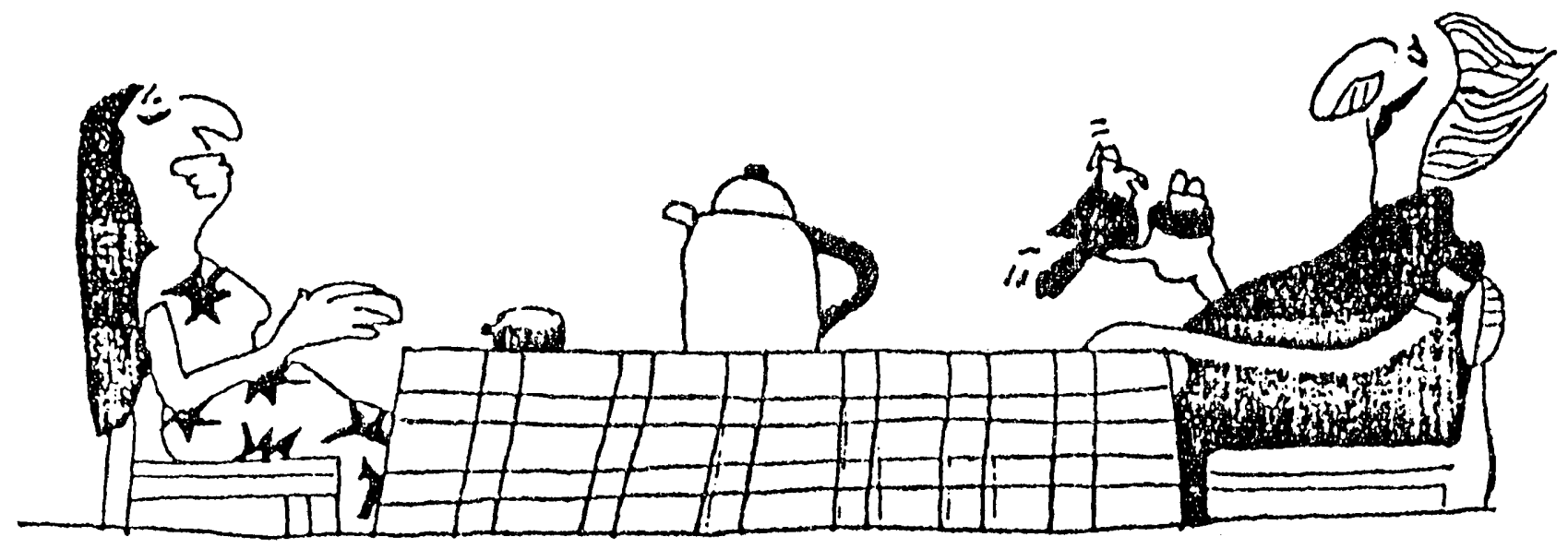

'UNNINESS =

VERSIVENESS =

ECAUSE. . . 


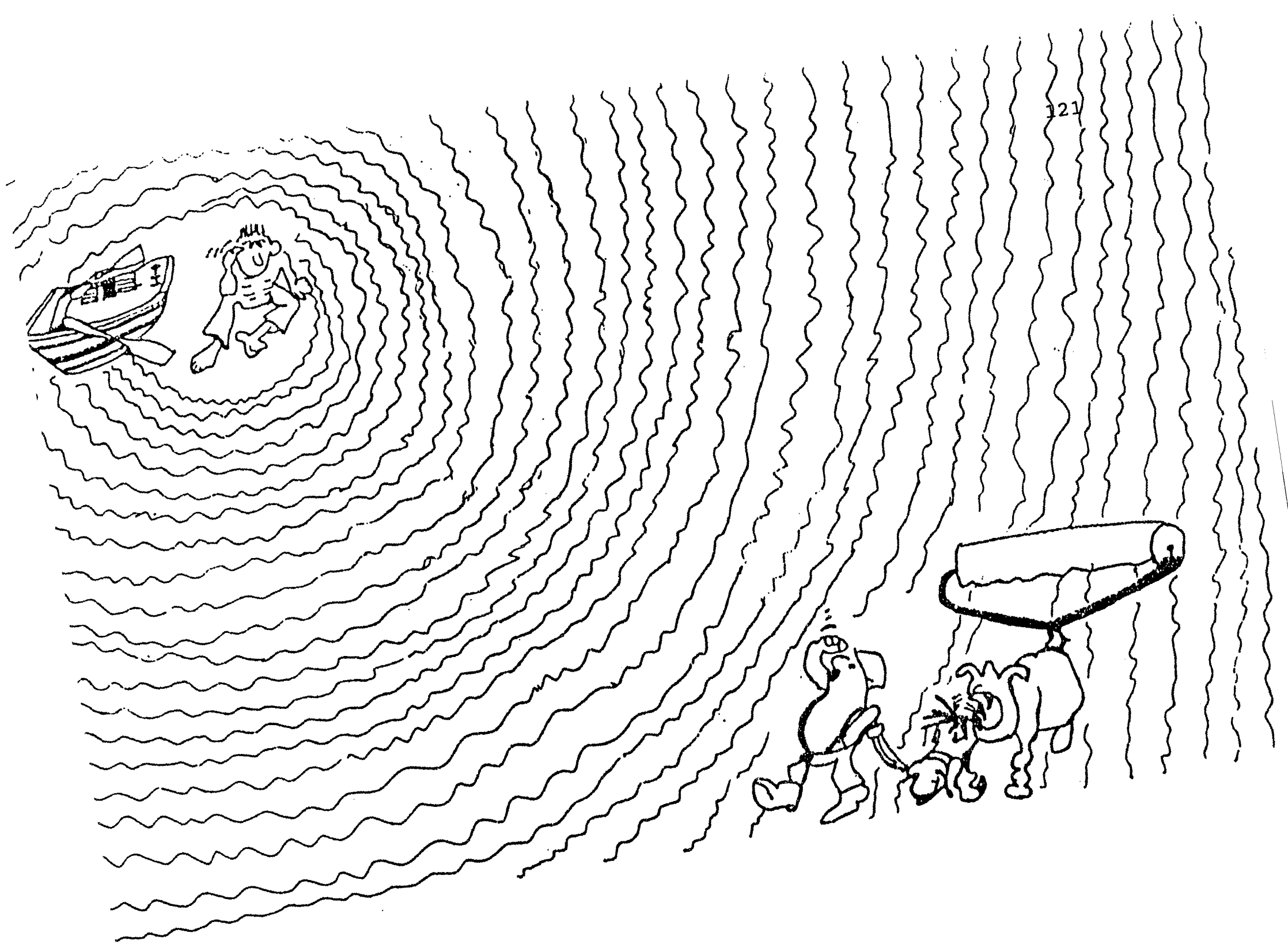

IINESS =

SIVENSS =

USE... 


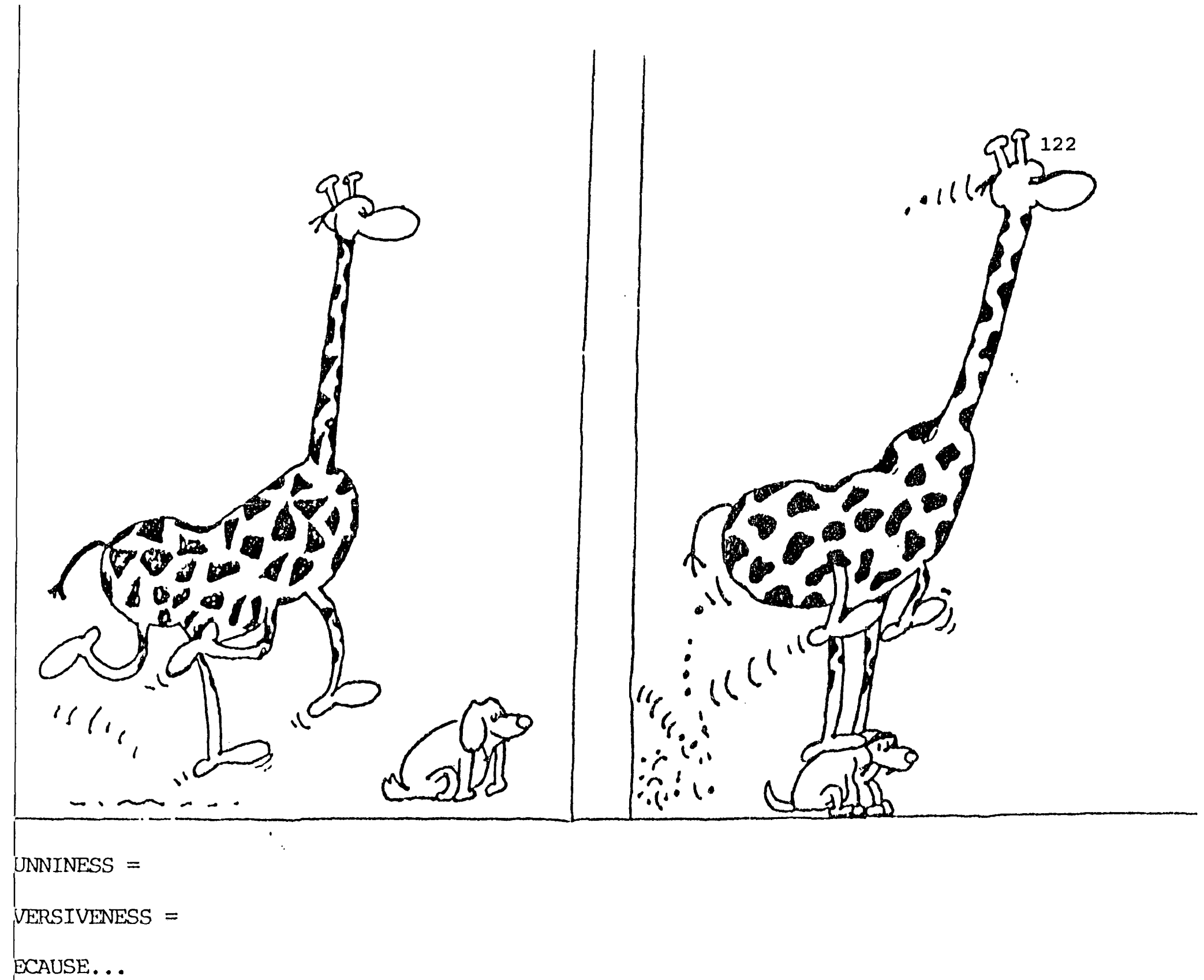




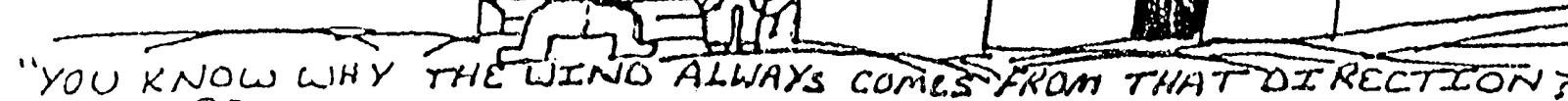
BECAUSE EAGLES. NEVES FKY CIAGKLLAOUS."

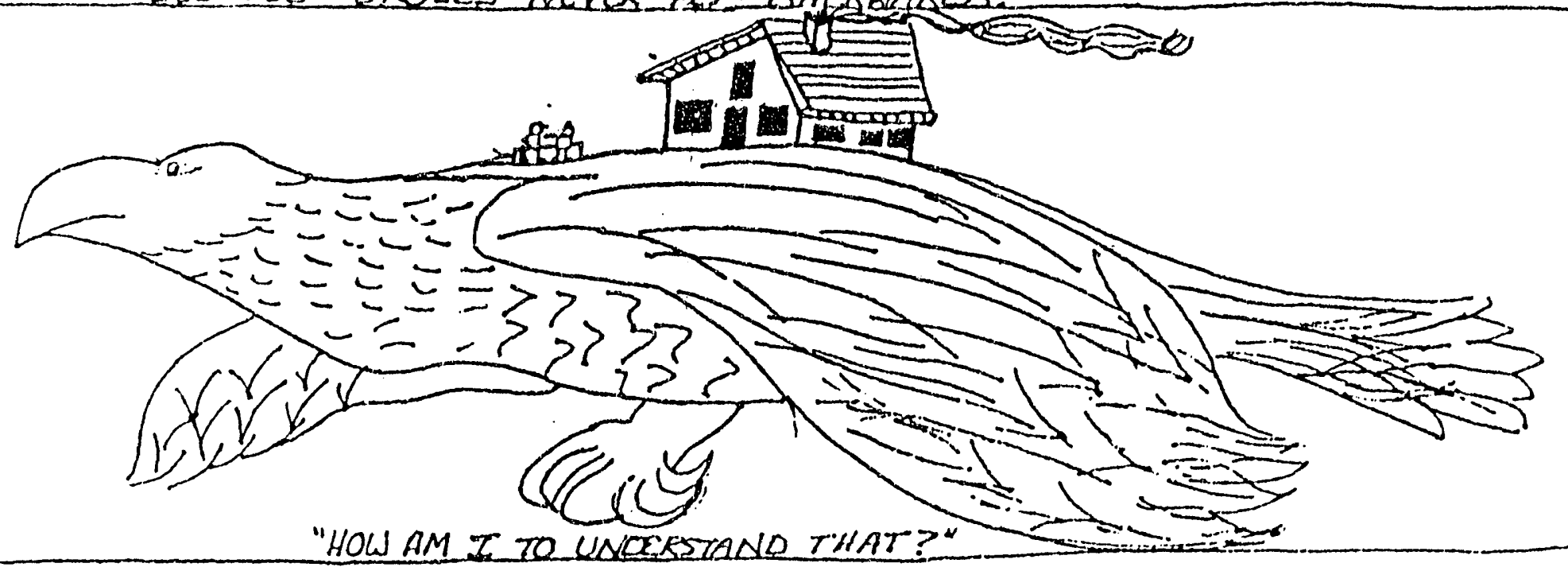

ININESS =

RSIVENESS =

AUSE... 


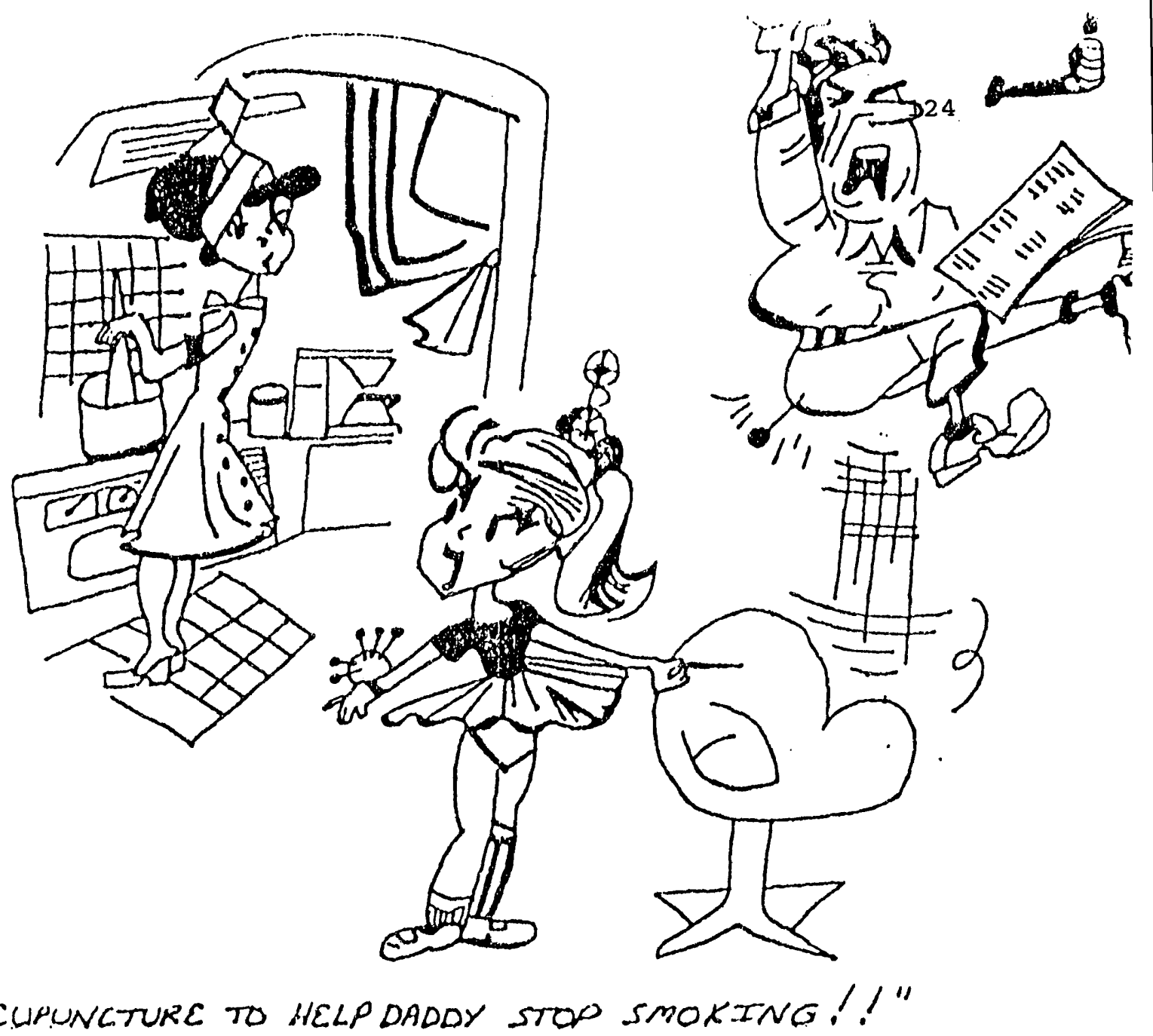

ININESS =

RSIVENESS =

AUSE... 


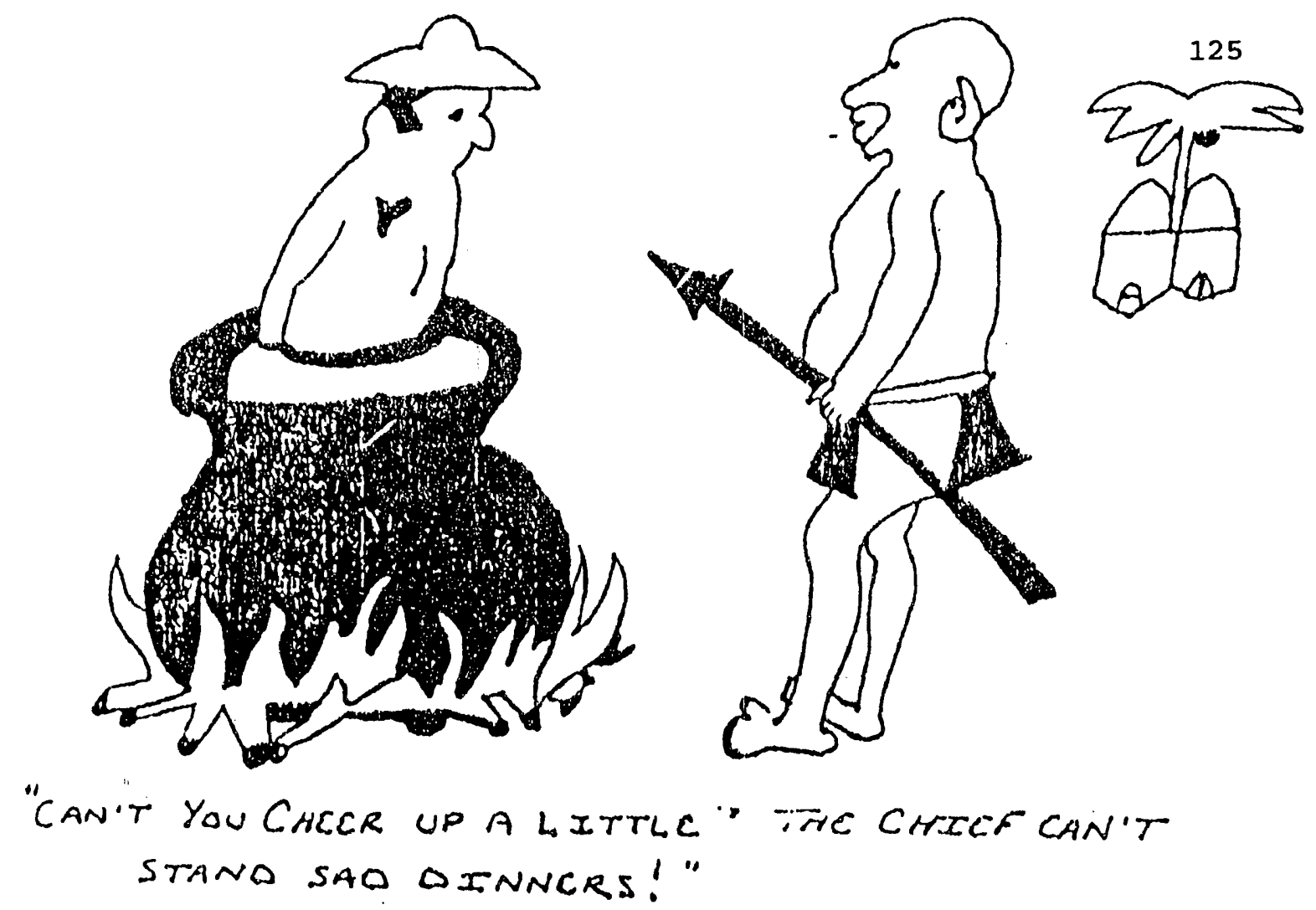

FUNNINESS = AVERSIVENESS = BECAUSE. . . 


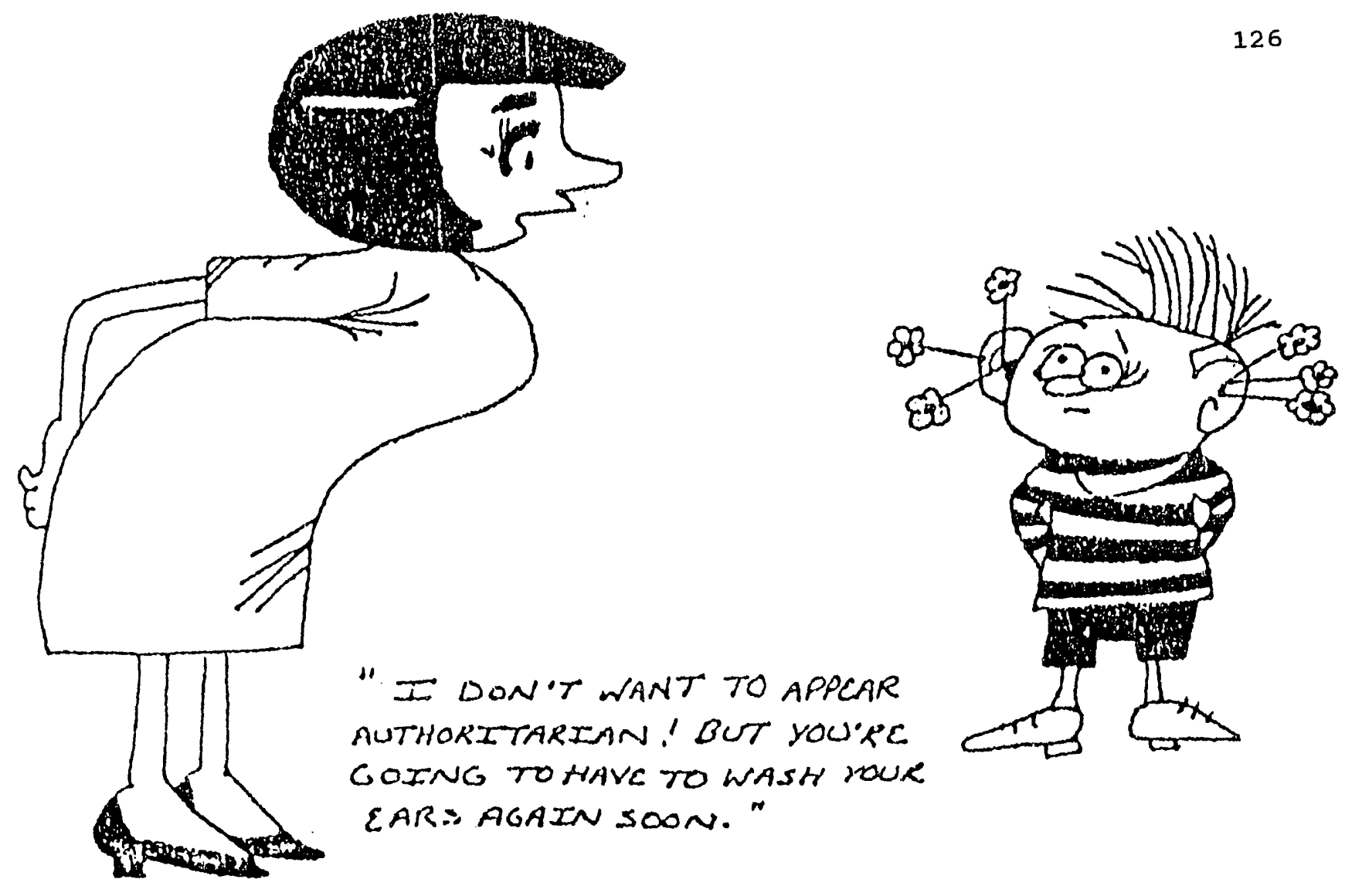

JNNINESS =

VERSIVENESS =

ECAUSE...

it in 


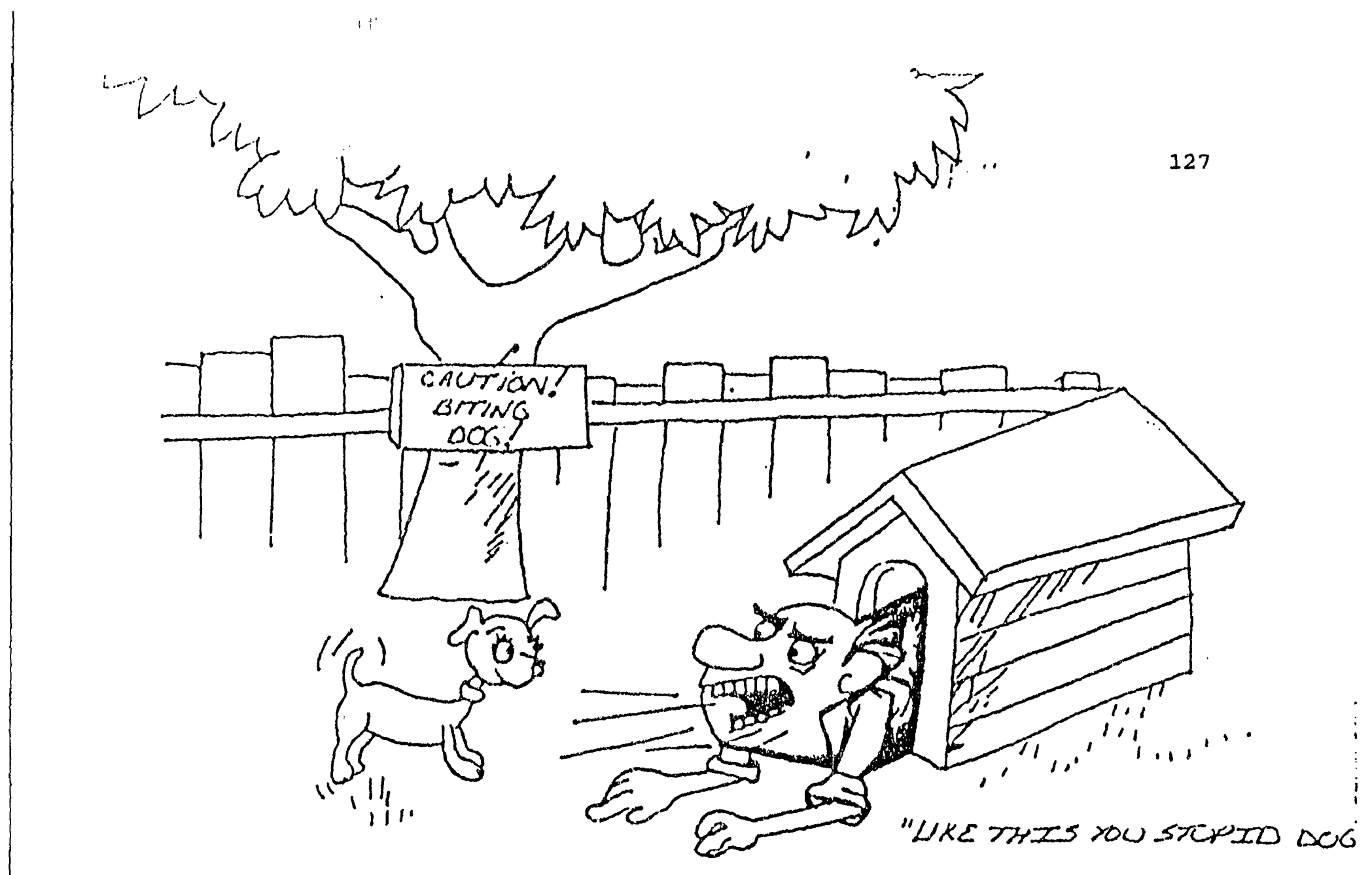

NNINESS $=$

ERSIVENESS =

XAUSE...

in 
JOKE OF THE MONTH

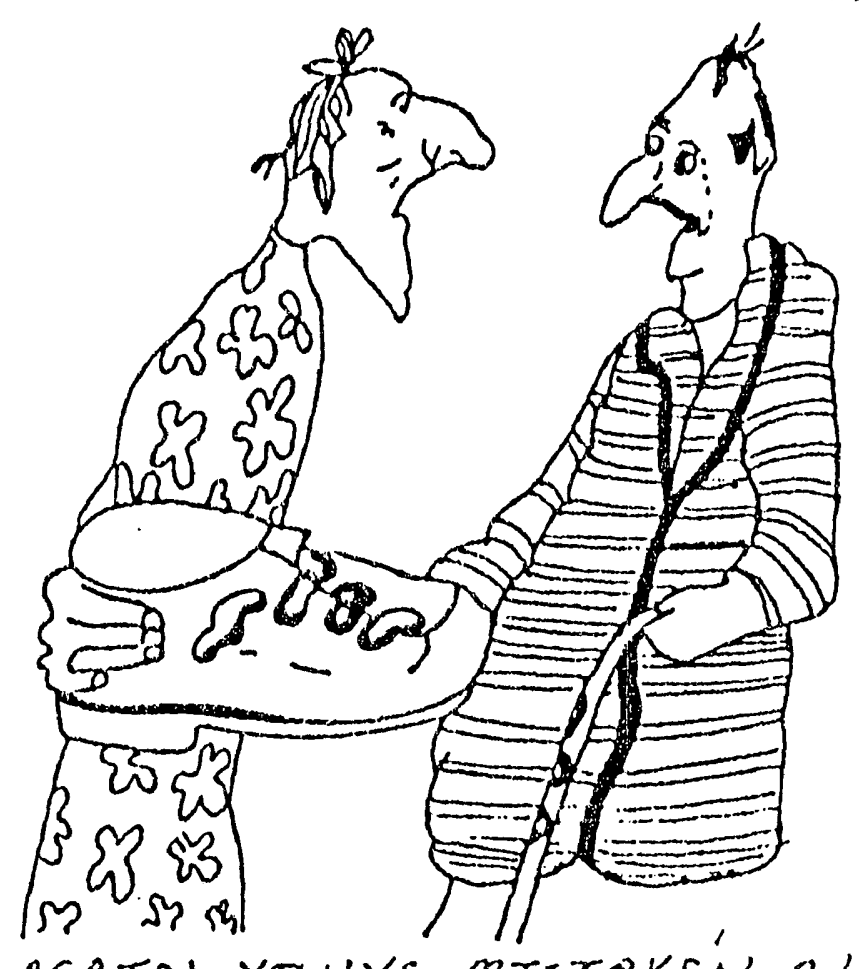

" once again you.ve mistrikeń a wokm FOR YOUR SHOELACE ORANDPA"

DNNINESS $=$
TERSIVENESS $=$ XCAUSE... 
WORK, Work...

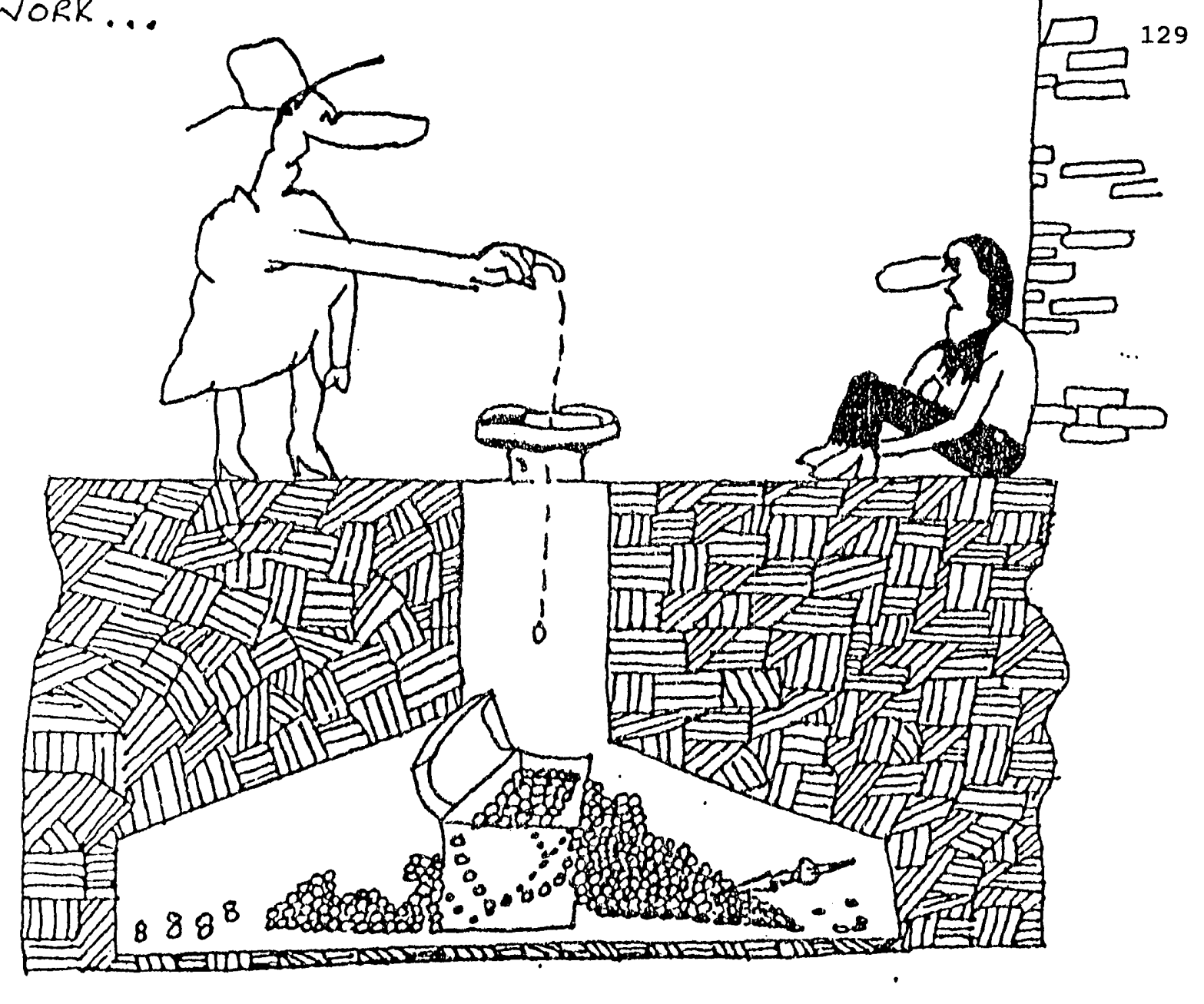

FUNNINESS =

AVERSIVENESS =

BECAUSE. . . 


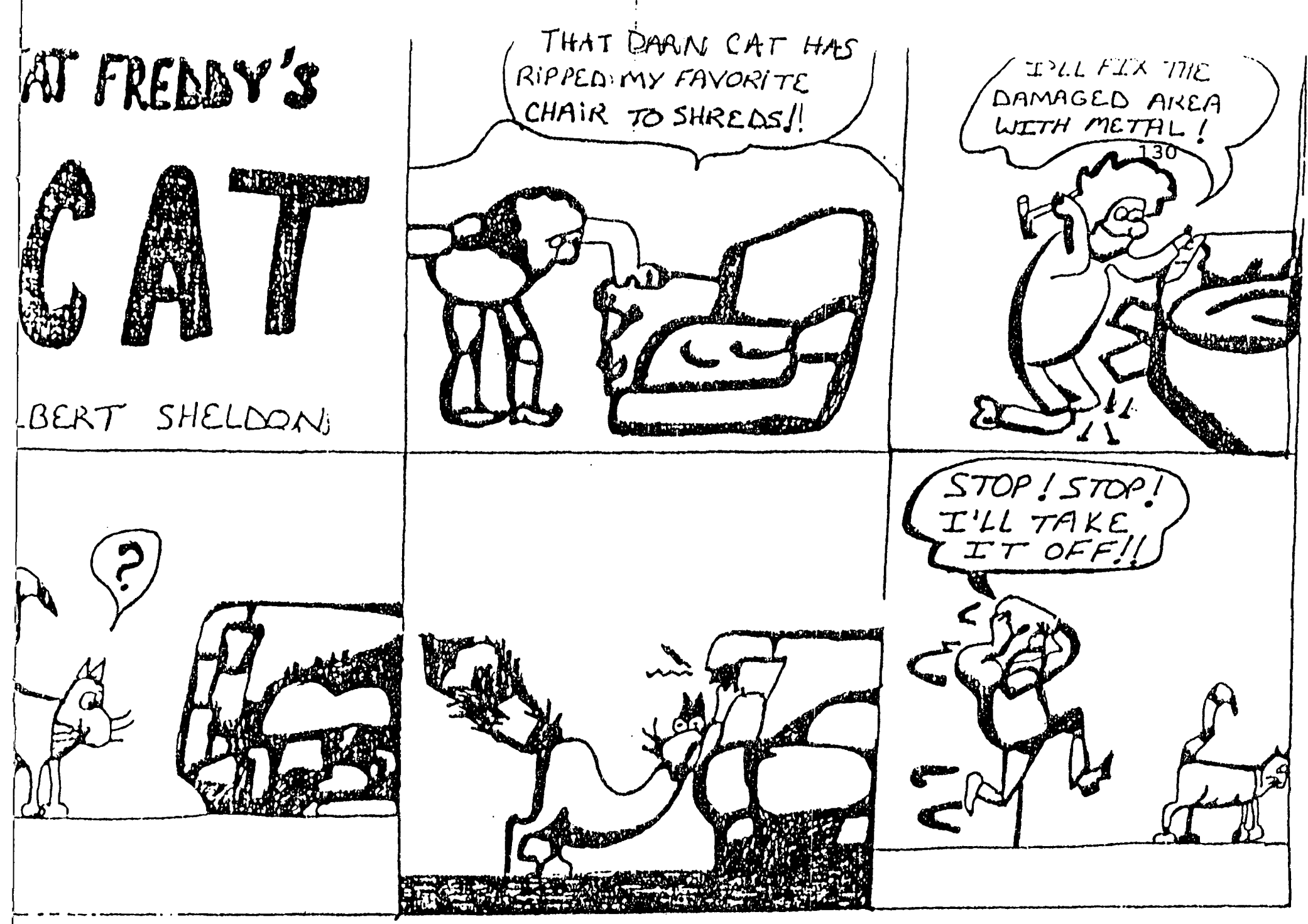

FUNNINESS $=$

AVERSIVENESS = BECAUSE. . . 
VITA

Rosemary Evans sțaley

The author was born in Oxford, England on 16 september 1964. She graduated from Gloucester High School, Gloucester, Virginia, in June, 1982. She received her Bachelor of Arts in Philosophy in December, 1986 from the College of William and Mary, in Virginia and entered the Master of Arts program in Psychology at the College of William and Mary, in Virginia, August, 1991. After receiving her Master of Arts in May, 1993, she will enter the Doctor of Philosophy program in Psychology at Georgetown University in Washington, the District of Columbia, beginning August, 1993. 